\title{
THE EFFECT OF FABRIC AND FIBER TOW SHEAR ON DUAL SCALE FLOW AND FIBER BUNDLE SATURATION DURING LIQUID MOLDING OF TEXTILE COMPOSITES
}

\author{
by \\ Joseph R. Walther
}

\begin{abstract}
A thesis submitted to the Faculty of the University of Delaware in partial fulfillment of the requirements for the degree of Master of Science in Mechanical Engineering
\end{abstract}

Summer 2011

Copyright 2011 Joseph R. Walther All Rights Reserved 


\section{THE EFFECT OF FABRIC AND FIBER TOW SHEAR \\ ON DUAL SCALE FLOW AND FIBER BUNDLE \\ SATURATION DURING LIQUID MOLDING \\ OF TEXTILE COMPOSITES}

by

Joseph R. Walther

Approved:

Suresh G. Advani, Ph. D.

Professor in charge of thesis on behalf of the Advisory Committee

Approved:

Anette M. Karlsson, Ph. D.

Chair of the Department of Mechanical Engineering

Approved:

Babatunde A. Ogunnaike, Ph. D.

Interim Dean of the College of Engineering

Approved:

Charles G. Riordan, Ph.D.

Vice Provost for Graduate and Professional Education 


\section{ACKNOWLEDGMENTS}

This thesis would not have been possible without the guidance of my

advisor, Dr. Suresh Advani. Thank you for all of your direction, patience, and support over the past two years. I am also very grateful to Dr. Pavel Simacek for all of his supervision and assistance. It has been my pleasure to work with you both.

I would like to thank Dr. Shridhar Yarlagadda and Stephen Andersen for their continued support and assistance with the development of my career as an engineer.

I am very grateful to Ted Lake, Rennie Burris, and Mark Scott for all of their help and guidance over the past three years. In addition, I would like to thank everyone in the CCM family for making my time here so enjoyable and fulfilling.

I owe my deepest gratitude to my mom, Maryann, my dad, Steve, and my brother, Eric, for all of their love and support not only in my education, but in my personal growth as well.

I would also like to thank all of my friends from college who have stuck with me through thick and thin. I will forever value all of the laughs, all of the time we have shared, and all of the support you have given me over the years.

Partial support of the National Science Foundation (NSF) under grant number 0856399 is gratefully acknowledged. 


\section{DEDICATION}

To my mom, Maryann:

I am honored to dedicate my thesis to you. I would not be here, and none of this would have been possible if it were not for you. You instilled in me at an early age the work ethic and drive I needed to reach this point in my life. You always stressed the importance of hard work, education, and pride in one's self. I have strived to succeed not only for myself, but for you as well. Making you proud is one of the few ways I can repay you for all that you have done. Thank you for all of your hard work and the sacrifices that you made during my childhood to ensure that Eric and I wanted for nothing. I love you very much, and I am very grateful for all that you have done for me over the past twenty-five years.

Your grateful son,

Joe 


\section{TABLE OF CONTENTS}

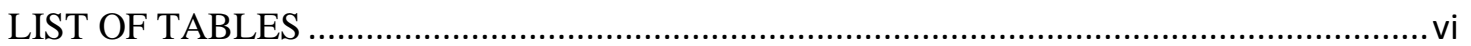

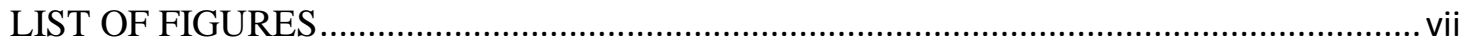

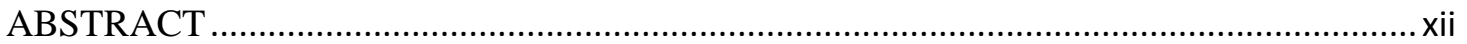

Chapter

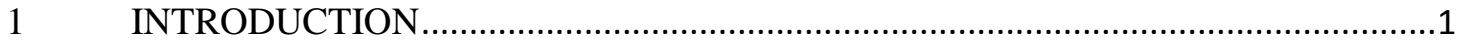

2 NUMERICAL MODELING .......................................................................

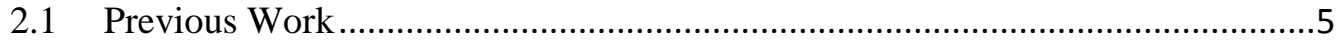

2.2 Modeling Shear Influenced Tow Saturation.......................................................14

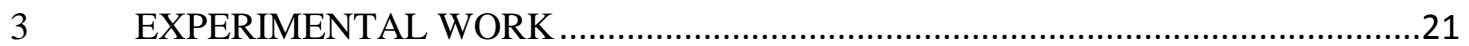

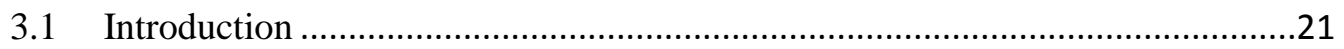

3.2 Preform Permeability as a Function of Fiber Volume Fraction ........................22

3.3 Fiber Tow Saturation and Permeability as a Function of Shear .........................28

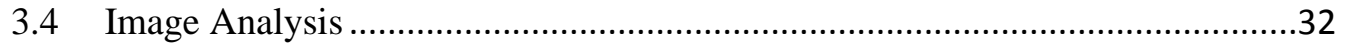

3.5 Resin Injection Experiments with Fabric Shear ............................................41

3.6 Bulk Permeability Characterization.................................................................55

3.7 Tow Permeability Characterization .............................................................61

$4 \quad$ INFLUENCE OF FIBER TOW SHEAR ON RESIN FLOW ……………………......68

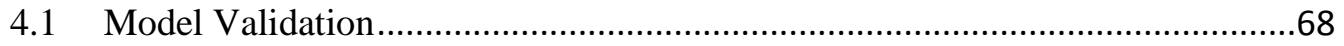

4.2 Quantifying the Influence of Fiber Tow Shear on Mold Filling .......................71

$5 \quad$ CONCLUSIONS, CONTRIBUTIONS AND FUTURE WORK …..........................95

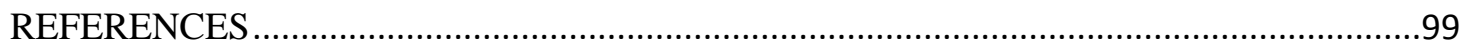

Appendix

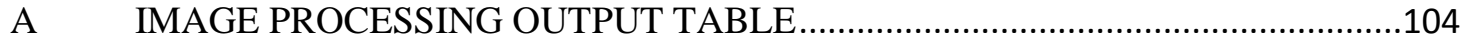

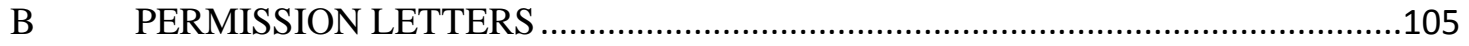

C FIBERSIM TO LIMS CONVERSION TOOL MANUAL .....................................111 


\section{LIST OF TABLES}

Table 1: Warp permeability as a function of fiber volume fraction ..................... 27

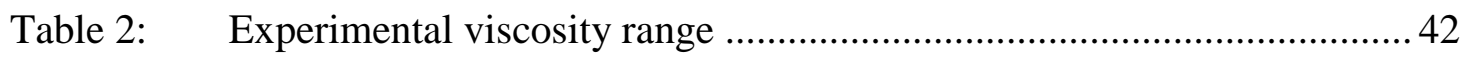

Table 3: $\quad$ Measured rotation of principal flow direction as a function of fabric shear angle........................................................................ 47

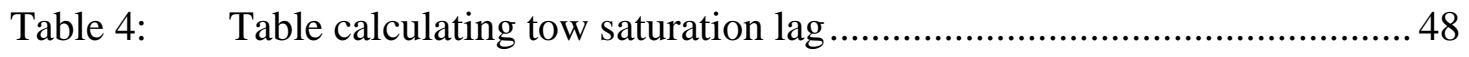

Table 5: Harmonic means and averages of tow saturation lag as a function of shear angle once the saturated flow lag becomes constant ................52

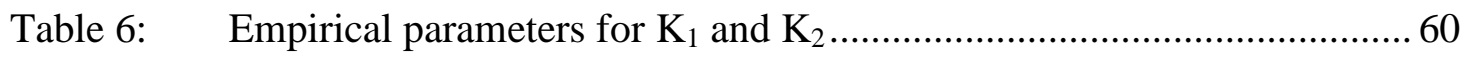

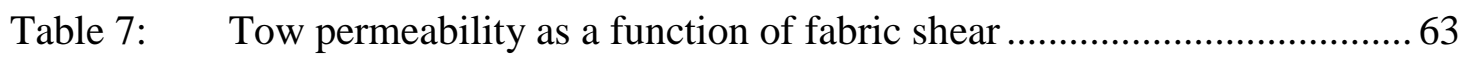

Table 8: Change in tow saturation lag versus shear angle for set reductions

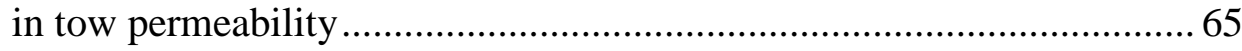

Table 9: Empirical permeability parameters used for principal fabric $\left(\mathrm{K}_{1}\right.$, $\mathrm{K}_{2}$ )and fiber tow $\left(\mathrm{K}_{\mathrm{tow}}\right)$ in the numerical model simulations .................6 68

Table 10: Average difference between witnessed experimental and simulation fill times

Table 11: A comparison of the amount of unfilled preform versus time for the three simulation types of interest. The amount of unfilled volume is recorded every 100 seconds for each simulation to demonstrate how the simulation that accounts for the reduction in tow permeability (iii) fills slower than its counterparts.

Table 12: $\quad$ Percentage of unfilled hemispherical preform comparison for the four different model simulations

Table 13: Percentage of unfilled preform comparison for the four different conical model simulations

Table A.1: Table output by image processing code 104 


\section{LIST OF FIGURES}

Figure 1: Various dual-scale fabric architectures. Note the spaces between the fiber tows is much larger than between the fibers, hence the permeability of fiber tows is usually much lower $[9]$............................ 2

Figure 2: $\quad$ Sheared orthogonal fabric and unit cell [29] ........................................... 6

Figure 3: $\quad$ Rotated flow ellipse due to increased fabric shear during resin injection [32] ........................................................................ 8

Figure 4: (a) Idealized (Top) and (b) dual scale flow in textile preforms (Bottom) [35]

Figure 5: (a -left) Addition of fiber tow internal volume externally by attachment of one-dimensional "slave" elements to all the nodes of an original "master" element (quad in this case) and (b-right) changes in mesh to model saturation of dual-scale porous medium. [35]

Figure 6: $\quad$ Rectangular cross-section of fiber tow ................................................... 13

Figure 7: (a - left) Layup reference directions showing warp, weft, and $45^{\circ}$ directions and (b - right) Local shear angle for the finite element draping simulation increasing from no shear (blue) to high local shear (red) 16

Figure 8: Flow chart delineating steps in the modeling process ............................ 17

Figure 9: Shows a reduction in tow permeability with an increase in fabric shear

Figure 10: Demonstration of the similar optical properties of glass fibers and vinyl ester resin. As the two are combined and the glass fiber tow wets out, it becomes translucent [41]. ...................................................... 22

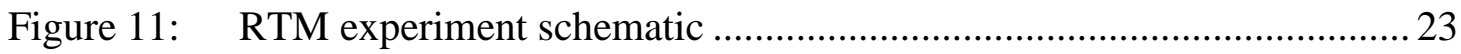

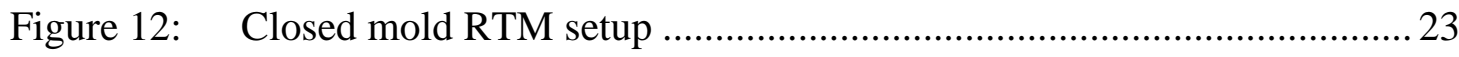

Figure 13: Resin inlet and air channel in RTM experiment setup ............................25 
Figure 14: Tracking of fluid flow front for 1D flow experiment.

Figure 15: The slope of square of the resin flow front position as a function of time when resin is injected under constant pressure is related to the permeability of the preform 26

Figure 16: Average warp permeability as a function of fiber volume fraction .......28

Figure 17: (a - left) A diagram of the experimental setup used by [37] to capture tow saturation data for the one-dimensional flow experiment shown in (b - right)

Figure 18: $(\mathrm{a}-\mathrm{left})$ The aluminum shearing fixture used throughout the designed experiment and (b) four layers of Owens Corning 24.5 oz. plain weave ShieldStrand-S fabric sheared to $20^{\circ}$.... 30

Figure 19: (a - top left) The shearing fixture is centered over a clear 12 inch square that represents the region of interest where video data will be collected, ( $b$ - top right) the sheared fabric and fixture laid up on the tool surface in a VARTM setup, (c - bottom left) the vacuum bagged experimental VARTM setup, and ( $\mathrm{d}$ - bottom right) a radial flow experiment underway.

Figure 20: (a - left) Radial flow experiment from a top and (b - right) bottom view.

Figure 21: Original raw experimental image cropped and converted to an intensity image for processing.

Figure 22: Image background continually grows darker due to increased light exposure as time progresses and more glass fibers are saturated.

Figure 23: Correction of image intensity drop for each image by subtracting a mean base intensity value.

Figure 24: (a - left) Intensity histogram displaying the image's average column vector intensity that is used to calculate the thresholding values to detect flow patterns such as the one shown in $(b-$ right $)$....... 36

Figure 25: (a - right) Application of the 9-block averaging smoothing function, (b - middle) the removal of all objects under 1000 square pixels, and (c) the resulting clean binary image. 
Figure 26: The four different image outputs from the image processing code for visual inspection and comparison of the results.

Figure 27: Best fit ellipses of logical images for both the saturated and macro flow front regions.

Figure 28: Determination of image scale 40

Figure 29: Flow front position vs. time for (a - left) the undeformed fabric and ( $\mathrm{b}$ - right) $30^{\circ}$ shear. There is a visually noticeable contrast of the gap between the macro flow front and the saturated fiber region.

Figure 30: Resin viscosity does not appear to have an effect on the tow saturation lag experienced during the experimental trials.

Figure 31: Difference in flow profiles for low (left) and medium (right) viscosity trials

Figure 32: Multiple flow ellipses seen due to interlayer ply slippage 45

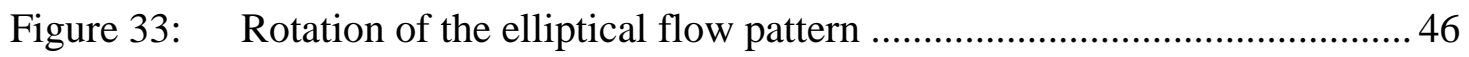

Figure 34: $\quad$ Rotation of principal flow direction by angle $\beta$.............................. 47

Figure 35: The linear nature of the unsaturated flow front lengths squared

Figure 36: Tow saturation lag vs. time for a single experiment of $30^{\circ}$ induced fabric shear....

Figure 37: Anisotropy ratio of principal permeabilities as a function of fabric shear

Figure 38: Experimental data for tow saturation lag vs. induced shear angle. .......53

Figure 39: Non-dimensionalized plot of tow saturation lag data............................ 54

Figure 40: Kozeny-Carmen equation fit to the experimental data.........................57

Figure 41: Calculated bulk principal permeabilities for a range of fabric shear angles for $24.5 \mathrm{oz}$ ShieldStrand-S fabric

Figure 42: Correlation between calculated permeability values and the proposed empirical model data. 
Figure 43: Anisotropic relationship between the principal permeabilities increasing as a function of fabric shear

Figure 44: $\quad(a-$ left $)$ Fine mesh used to track tow impregnation and $(b-$ right $)$ mold filling times predicted by the finite element simulation. 62

Figure 45: Tow permeability as a function of fabric shear angle ............................ 64

Figure 46: Change in tow saturation lag as tow permeability is varied.................... 66

Figure 47: Correlation between calculated tow permeability data and the proposed fit model values

Figure 48: Comparison between experimental and simulation flow fronts for set time steps. All images represent flow in an approximately 3" square.

Figure 49: (a - left) Location of injection gate for radial flow simulation and $(b-$ right) radial flow front progression vs. time. Flow ellipse rotated due to induced shear angle

Figure 50: Influence of shear angle on LCM simulations. Baseline case assumes that tows and intertow regions fill at the same rate. No tow shearing case assumes that tows fill at much slower rate according to Figure 4. Tow shearing case results in even lower permeability needing extra time to completely fill the sheared tows.

Figure 51: Fraction of unfilled preform as a function of time for simulations when the preform was subjected to a shear angle of $35^{\circ}$ 76

Figure 52: Comparison of tow saturation flow front for simulations (i), (ii) and (iii) to observe the influence of tow deformation of filling 78

Figure 53: From left to right, a CAD model of tool surface, the draping simulation showing fabric shear zones, and the final 3D mesh in LIMS 79

Figure 54: Fluid injection simulation in a hemispherical mold 80

Figure 55: The draping simulation performed on the hemisphere mold surface, and the resulting 3D element FVF and tow element permeabilities 
Figure 56: Comparison of simulation techniques and their corresponding flow fluid flow fronts

Figure 57: Comparison of the total hemispherical preform fill fraction versus time for each model simulation

Figure 58: Inlet pressure profile comparison to fill the first 95\% of control volume

Figure 59: The drastic increase in pressure required to drive the flow for the last $1 \%$ of $\mathrm{CV}$ for the case when tow shearing is present ....................... 88

Figure 60: Finite element flow simulation of a conical preform ............................. 89

Figure 61: From left to right: FiberSIM draping simulation over the cone surface, the macro element FVF, and the resulting tow element permeabilities 90

Figure 62: Comparison of fluid flow fronts through the cone preform for the three different model types

Figure 63: Comparison of the total preform fill fraction versus time for each conical model simulation 


\begin{abstract}
In Liquid Composite Molding (LCM) processes, a fibrous reinforcement preform is placed or draped over a mold surface, the mold is closed and a resin is either injected under pressure or infused under vacuum to cover all the spaces in between the fibers of the preform to create a composite part. LCM is used in a variety of manufacturing applications, from the aerospace to the medical industries. In this manufacturing process, the properties of the fibrous reinforcement inside the closed mold is of great concern. Preform structure, volume fraction, and permeability all influence the processing characteristics and final part integrity. When preform fabrics are draped over a mold surface, the geometry and characteristics of both the bulk fabric and fiber tow bundles change as the fabric shears to conform to the mold curvature. Numerical simulations can predict resin flow in dual scale fabrics in which one can separately track the filling of the fiber tows in addition to flow of resin within the bulk fabric. The effect of the deformation of the bulk fabric due to draping over the tool surface has been previously addressed by accounting for the change in fiber volume fraction and permeability during the filling of a mold. In this work, we investigate the effect of shearing of the fiber tows in addition to bulk deformation during the dual scale filling. We model the influence of change in fiber tow characteristics due to draping and deformation on mold filling and compare it with the results when the fiber tow deformation effect is ignored. Model experiments are designed and conducted with a dual scale fabric to characterize the change in permeability of fiber tow with deformation angle. Simulations which account for dual scale shear demonstrate that
\end{abstract}


the tow saturation rate is affected, requiring longer fill times, or higher pressures to completely saturate fiber tows in areas of a mold with high local shear. This should prove useful in design of components for applications in which it is imperative to ensure that there are no unfilled fiber tows in the final fabricated component. 


\section{Chapter 1}

\section{INTRODUCTION}

In liquid composite molding (LCM), resin is injected into a preform containing many different possibilities for reinforcement. Whether the preform is composed of chopped fiber mat, different core sections, or woven fabrics, the preform permeability is of great concern to engineers and scientists responsible for designing composite parts and manufacturing processes. Many researchers [1-4] have developed ways to experimentally determine and numerically model the permeability and flow characteristics of such preforms. Once the material properties are characterized, they can be used in mold filling simulations to predict the impregnation of preforms with resins to ensure that the resin will cover all the empty spaces between the fibers tows and in regions between the fibers within fiber tows. Many such simulation packages have been developed over the last two decades [1-2, 5].

With these numerical simulations, engineers can predict how resin will flow in a closed mold as long as material properties such as fiber volume fraction, and the permeability values of the permeability tensor of a fabric is determined theoretically or experimentally for the specific fabric being used. The simulation can usually track flow front progression based on the permeability values provided every element in the geometric mesh and therefore can be used to locate the last regions to fill, develop methods to explore optimal placement of gates and vents, use them to design the part and the process and also introduce control to improve productivity 
[4, 6-8]. This virtual manufacturing greatly reduces the amount of wasted resources in time and capital needed for trial and error manufacturing, making composites processing much more economic.
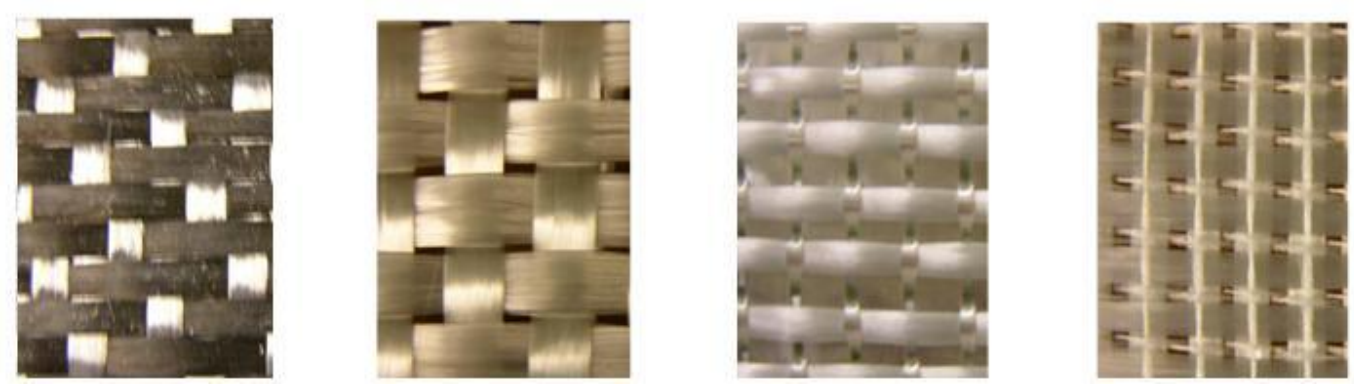

Figure 1: Various dual-scale fabric architectures. Note the spaces between the fiber tows is much larger than between the fibers, hence the permeability of fiber tows is usually much lower [9]

The simulation results will be accurate only if the input material properties realistically describe the preform characteristics. Most textile fabrics are either woven or stitched as shown in Figure 1. These fabrics exhibit a dual scale structure. The fiber tows which may consist of 100 to 12000 individual filaments are of the order of millimeters as compared to the individual filaments which are of the order of micrometers. Hence the gaps between the fiber tows are much larger as compared to the gaps between individual fibers as can been seen from Figure 1. Thus the permeability of the fabric in between the fiber tows is going to be much higher than the permeability of the fiber tows as measured by various researchers [5, 8, 10-12]. Due to this dual scale nature, the resin fills the gaps in between the tows much before it fills the fiber tows. This has been numerically modeled by the simulation package 
developed at the University of Delaware called Liquid Injection Molding Simulation [1].

In most liquid molding processes, the woven or stitched fabric needs to be draped over the tool surface. As a fabric is draped over a curved surface of a mold, the fabric architecture is deformed. This local deformation leads to changes in fabric geometry, volume fraction and in direction and magnitude of the principal components of the permeability tensor that will influence the mold filling process and also the mechanical properties. The main type of deformation that occurs during draping is fabric shear. Many researchers [13-21] have dedicated their efforts to develop models to predict the behavior of fabrics under certain deformation schemes. From the overall deformations, there have been models developed to predict the change in permeability as a function of deformation [13-20]. This modified information is then used in the flow models to track the movement of the flow front [18-21].

However, all the models that predict permeability as a function of deformation have focused on finding the change in effective permeability in the local region. They do not account for separate change in permeability of the fiber tows due to the deformation as one would expect for a dual scale fabric. It has been shown by Badel et. al [22] that fiber tow geometry is changed during fabric shearing. The change in tow geometry will lead to varying fiber bundle porosity and permeability, thus changing the permeability of the tow and the time it takes to fill a fiber tow and thus the processing characteristics of the preform. The focus and contribution of this thesis is to model the influence of change in fiber tow characteristics due to draping and deformation on mold filling and compare it with the results that ignore this effect to gauge the importance of including this effect when addressing the filling of textile 
preforms that are draped over a compound surface. Three important assumptions made in this modeling are (i) surface tension effects of the resin are ignored and (ii) the fiber tow permeability is assumed to be transversely isotropic, and (iii) all of the flow saturating the fiber tows is in the transverse direction and hence, the longitudinal permeability is negligible. The first assumption breaks down when the flow becomes very slow when filling the fiber tow as flow due to the capillary effects will become comparable. The second assumption is reasonable if one assumes that flow that resists filling is primarily in the transverse direction to the direction of the fiber tows which stems from the geometric arrangement of the tows. The third assumption is justifiable when considering the tow length scaling or distance for full fluid impregnation of the saturating flow. 


\section{Chapter 2}

\section{NUMERICAL MODELING}

\subsection{Previous Work}

It has been shown that fabric deformation due to draping changes the permeability and hence the resistance to flow and the processing characteristics of the fabric. Experiments have been designed and conducted to determine the local change in principal flow directions, fiber volume fraction (FVF), and permeability for different fabrics [23-27]. This information coupled with a finite element draping simulation, as was performed in $[18,21]$, can lead to a more realistic numerical model that is capable of predicting flow fronts and fill times for draped fabrics, as can be seen in Louis and Huber [18]. These draping simulations, usually based on kinematics, output a fabric shear angle $\alpha$ for each finite element zone or element. From the angle one can calculate the local FVF for each element using the following equation:

$$
v_{f}=\frac{v_{f o}}{\cos \alpha}
$$

where $v_{f}$ is the local FVF, $v_{f o}$ is the original bulk fabric FVF, and $\alpha$ is the shear angle determined by the draping simulation. This equation was determined from the geometrical analysis of a sheared unit cell as discussed by Skelton [28] and assumes no slippage between fiber tows as well as no fabric wrinkling as shown in Figure 2 below. This is of course theoretical and does not account for fiber tow slippage at 
extreme shear angles. Also, there exists a critical shear angle based upon the initial undeformed fiber volume fraction which will predict deformed fiber volume fractions of one hundred percent, or greater. This of course is unrealistic, and the numerical model must be adjusted in a manner that conserves porous volume and agrees with empirical results.

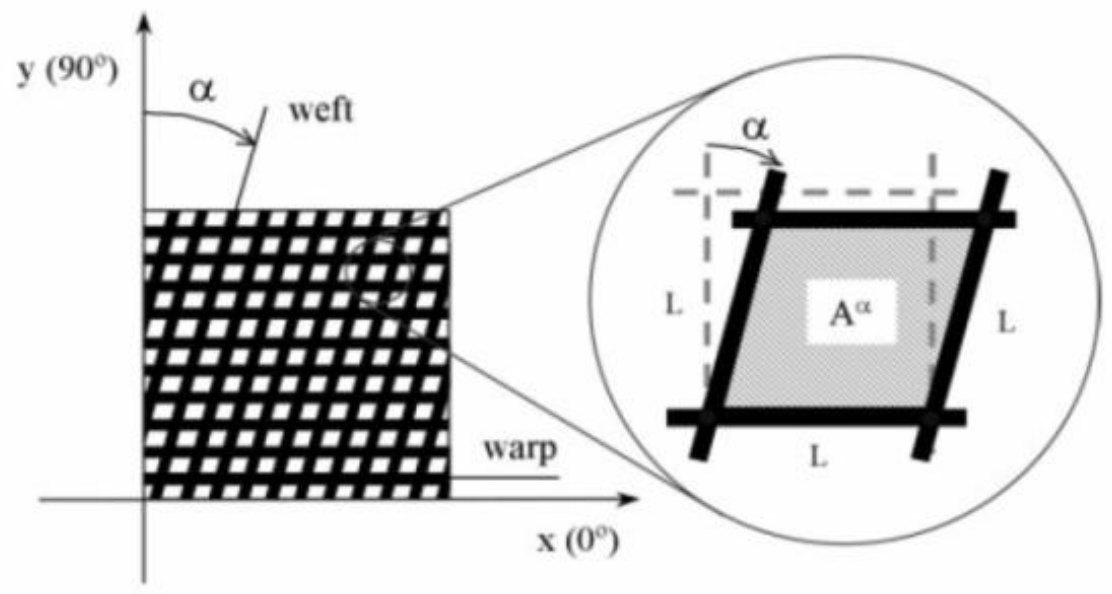

Figure 2: Sheared orthogonal fabric and unit cell [29]

Permeability of a fabric is a second order tensor. A planar fabric will have three independent components $\left(K_{11}, K_{22}\right.$ and $\left.K_{12}=K_{21}\right)$. When the principal flow direction is along the warp direction of the fabric, $K_{11}=K_{\text {warp }}, K_{22}=K_{\text {weft }}$ and $K_{12}=0$. When $K_{12}$ is not zero, the flow direction is at an angle to the warp and weft direction. Usually change in fiber volume fraction will cause the principal permeability values to change. Many models, such as the Kozeny-Carman equation discussed in [14], have 
been developed as general models that account for change in principal permeability values as a function of fiber volume fraction:

$$
K=\frac{r_{f}^{2}}{4 k} \frac{\left(1-v_{f}\right)^{3}}{v_{f}^{2}}
$$

where $r_{f}$ is the radius of the fiber tows and $k$ is the Kozeny constant. As discussed in [14], $r_{f}^{2} / 4 k$ is determined from experimental data. There have been other models that also describe this change such as the one proposed by Gebhart and Brushke [30-31]. One can also determine the change in principal angles due to the shearing angle $\alpha$ by conducting a radial flow experiment and observing the rotation of the flow ellipse during resin injection due to shear [14-15, 18-21]. The change in the major and minor axis of the ellipse due to the shearing of the fabric can be measured. The ratio of the in-plane principal permeability tensor can also be determined from the same experiment by measuring the ratio of the major and minor axis for a fully developed elliptical shape of the resin impregnated into a preform as follows

$$
\frac{K_{11}}{K_{22}}=\left(\frac{a}{b}\right)^{2}
$$

where $a$ and $b$ are the respective major and minor axes of the ellipse and $K_{11}$ and $K_{22}$ are the principal permeabilities of the sheared preform in the directions of the semimajor and semi-minor axes of the flow ellipse. Thus knowing how the local fiber volume fraction, magnitude and direction of the permeability tensor change with the shear angle for each finite element, one can accurately predict resin flow in a sheared fabric using the flow simulations such as LIMS. This methodology and the influence 
of shearing on the flow has been extensively studied and verified by many research groups [13-21] and also implemented in some flow simulation packages.

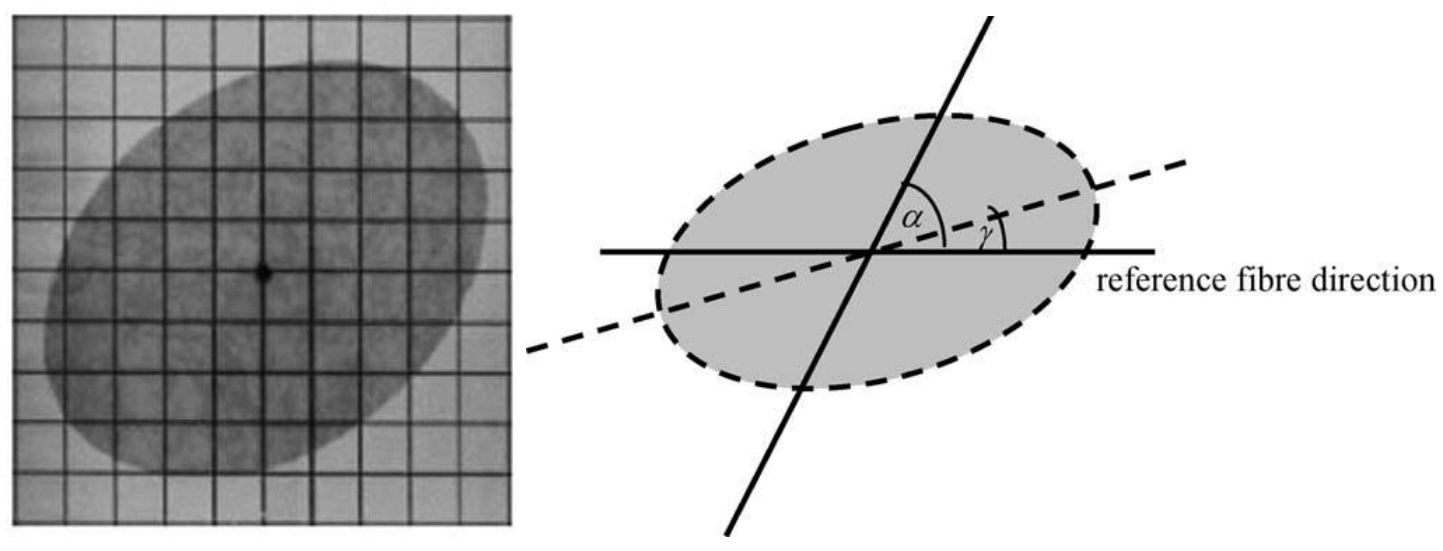

Figure 3: Rotated flow ellipse due to increased fabric shear during resin injection [32]

However, many traditional preforms in the automotive, aerospace, and structural sectors use orthogonal woven fabrics that need to be treated as dual scale porous medium, containing two distinct levels of channels for resin flow. The dual scale nature of these fabrics can be witnessed by the difference between the macroscopic inter-tow flow and the microscopic intra-tow flow. Unless spaces in between the fibers are not saturated with resin, the filling is not considered complete and the presence of such microgaps without resin in between fibers within a fiber tow can compromise the performance of the composite part. In the last decade, researchers have recognized the importance of dual scale flow and have developed models to 
address and predict fiber tow saturation along with bulk filling of the fiber preform [33-34].

When considering this microscopic flow, mass conservation (Eq. 4) is typically used to model flow if the tows fill at the same rate as the regions between the fiber tows,

$$
\nabla \cdot\langle v\rangle=0
$$

where $\langle v\rangle$ is the volume averaged velocity of the fluid flow. Darcy's law is used to describe the relationship between the resin flow, the pressure gradient $\nabla P$ and the permeability tensor of the fabric $\boldsymbol{K}$

$$
\langle\boldsymbol{v}\rangle=-\frac{\boldsymbol{K}}{\mu} \cdot \nabla P
$$

with resin viscosity $\mu$. Substitution of Eq. (5) into Eq. (4) results in the governing equation for pressure - which when solved allows one to predict flow in a single scale porous media or idealized dual scale flow as shown in Figure 4(a) as a function of fabric permeability and resin viscosity. 


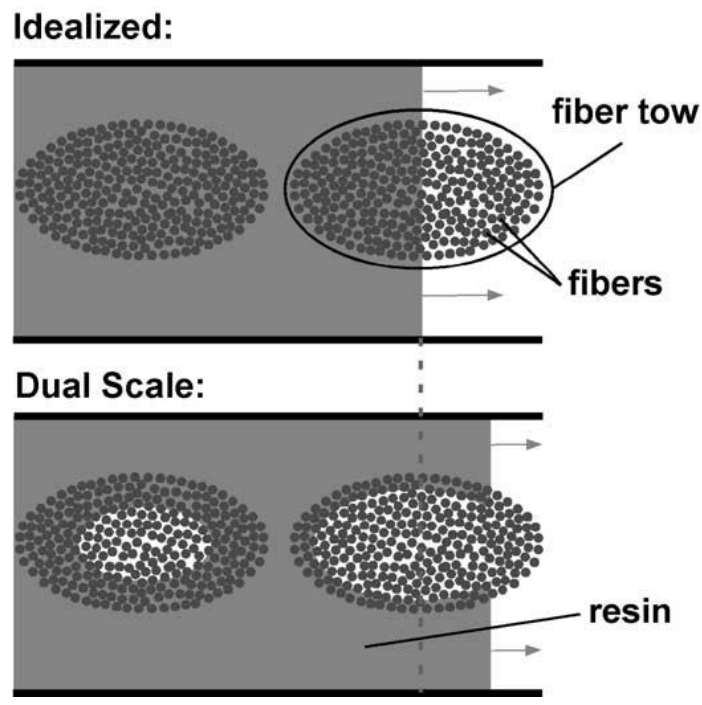

Figure 4: (a) Idealized (Top) and (b) dual scale flow in textile preforms (Bottom) [35]

However, for textile fabrics tow saturation occurs at a much slower rate than the macroscopic filling as shown in Figure 4(b), so it is necessary to accurately predict the saturation rate to allow the resin sufficient time to fully infiltrate the tows to ensure that a high quality part is produced. Schell et. al [36] as well as Zhou et. al [33] developed experiments to determine the transversal and longitudinal fiber bundle permeability. Neacsu et. al [37] then developed a set of experiments in which the saturation rate of glass fiber tows could be measured optically.

To address the modeling, a sink term is added to the governing continuity equation to account for the fluid flow in the tows of a dual scale porous media:

$$
\boldsymbol{\nabla} \cdot\left(\frac{\boldsymbol{K}}{\mu} \cdot \boldsymbol{\nabla} P\right)=q(p, s)
$$


Here $\boldsymbol{K}$ is the permeability tensor of the bulk fabric and the sink term $q$ is dependent on the surrounding fluid pressure $p$, the permeability of the fiber tow and the degree of tow saturation $s$ and one would need a model that can describe the relationship between the sink term and the pressure and state of saturation of the tow.

Simacek and Advani [34], instead of adding the appropriate sink term to the governing equations, added additional one-dimensional volume elements with properties associated with the fibrous tows which simplified the model. By attaching one-dimensional elements to the finite element mesh to represent fiber tows; the filling of these one dimensional elements can track fiber tow filling. Figure 5 (a) and (b) show the addition of one-dimensional bar elements that represent the volume within the fiber tows. This approach allowed them to separately report the filling of fiber tows along with bulk filling. The input required one to specify the fiber tow permeability for the one dimensional elements and bulk permeability for other elements. This thesis focuses on addressing the change in fiber tow properties due to shearing and the effect it has on filling of the tows which can be easily handled in the simulation LIMS once we can characterize the change in fiber tow permeability as a function of the deformation of the preform on the tool surface.

However, one needs to conserve the fiber volume and hence some book keeping needs to be done to redistribute the porous volume so the amount of space available for the resin remains the same with or without deformation within the mold. This can be expressed as shown in Eq. (7). Instead of reducing the size of the 3D elements which would require modifying the coordinates of all nodes, the porosity of the original 3D mesh elements can be reduced in order to account for the creation of this additional volume, which would model the fiber tows as porous structures. 


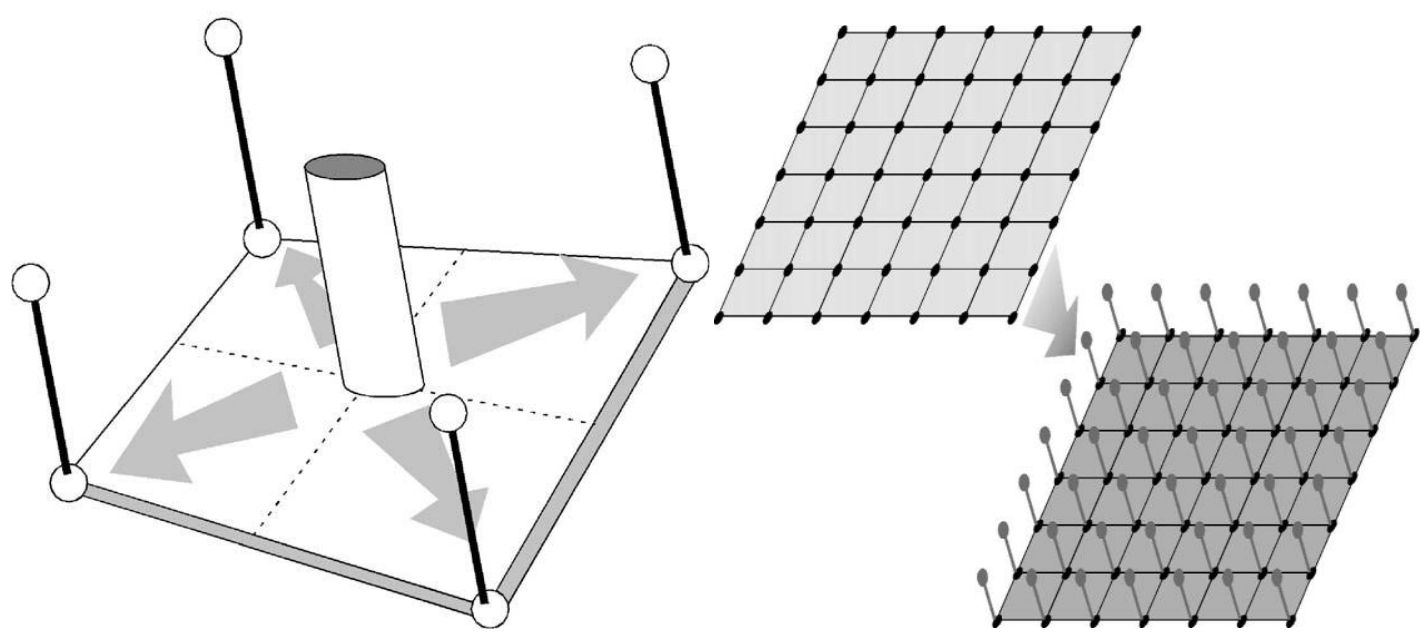

Figure 5: (a -left) Addition of fiber tow internal volume externally by attachment of one-dimensional "slave" elements to all the nodes of an original "master' element (quad in this case) and (b-right) changes in mesh to model saturation of dual-scale porous medium. [35]

The FEA control volume balance for the model now becomes:

$$
\emptyset V=\emptyset_{3 D} V_{3 D}+\emptyset_{\text {tow }} V_{\text {tow }}
$$

where $\emptyset$ and $V$ are the porosity and volume of the mold, $\emptyset_{t o w}, \emptyset_{3 \mathrm{D}}$ and $V_{\text {tow }}, V_{3 D}$ are the porosity and volume of the $1 \mathrm{D}$ tow elements and the 3D elements respectively. The volume of the mesh (mold) $V$ is determined by the thickness of the ply multiplied by its surface area. Using the initial fabric fiber volume fraction along with that of the fiber tows, one can determine the volume of the 1D tow elements:

$$
\begin{aligned}
& V_{\text {fiber }}=v_{f, \text { tow }} V_{\text {tow }}=v_{f} V \\
& V_{\text {tow }}=\frac{v_{f}}{v_{f, \text { tow }}} V
\end{aligned}
$$


Here $v_{f, t o w}$ is the fiber volume fraction of the fiber tow and $v_{f}$ is the fiber volume fraction of the bulk 3D elements. Note that the sum of porosity and fiber volume fraction is equal to unity. For simplicity, the size of the 3D mesh was unchanged, so $V_{3 D}$ is the same as $V$, and the new porosity of the 3D elements can be determined easily by substituting (9) into (7):

$$
\begin{aligned}
& \emptyset V=\emptyset_{\text {tow }} \frac{v_{f}}{v_{f, \text { tow }}} V+\emptyset_{3 D} V \\
& \emptyset_{3 D}=\varnothing-\emptyset_{\text {tow }} \frac{v_{f}}{v_{f, \text { tow }}}
\end{aligned}
$$

Now that the correct volume of the 1D tow elements has been determined from Eq. (9), the length and cross-sectional area must be established. As performed in Simacek and Advani [34], we assume that the fiber tow has a thin rectangular crosssection depicted in Figure 6 with width $2 b$, height $2 h$, and length $L_{l}$.

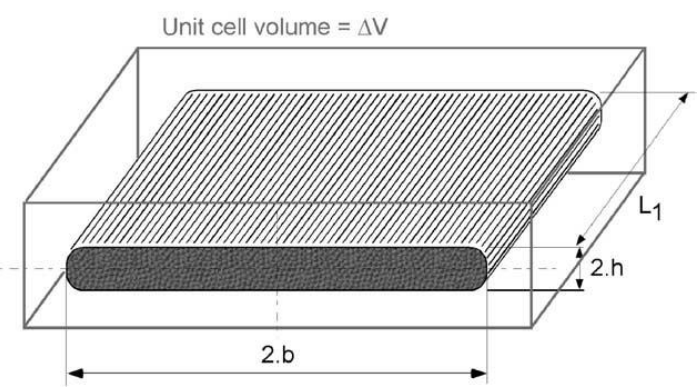

Figure 6: Rectangular cross-section of fiber tow [35]

During tow saturation, fluid travels a distance of $\mathrm{h}$ from top and bottom for full tow impregnation. Therefore we assume the length of the $1 \mathrm{D}$ tow elements $l=2 h$ because we must account for the fact that half of the 1D element belongs to the control volume 
of the base node that belongs to the 3D master element. Because the length of the $1 \mathrm{D}$ element is twice the length that fluid would need to travel, the effective element permeability is doubled to account for this assuming it fills according to Darcy's law. The cross-sectional area of the fiber tow elements can then be calculated by dividing the tow volume by the length of the $1 \mathrm{D}$ element. The $1 \mathrm{D}$ element properties can be expressed as follows:

$v_{f, \text { tow }}=$ fiber volume fraction of undeformed fiber tow

$K_{x x}=2 K_{\text {tow }} \quad$ (determined experimentally)

$l=2 h$

$A=\frac{V_{\text {tow }}}{l}$

where the one dimensional element effective permeability $K_{x x}$ is twice that of the experimentally determined tow permeability $K_{\text {tow }}$. We now have a finite element mesh that can simulate the two different macro and micro flows that occur in a dual scale porous media. This dual scale flow has been modeled in undraped fabrics using LIMS [34]. The next section will investigate how the change in permeability of $1 \mathrm{D}$ tow elements and the bulk 3D mesh elements can be adjusted due to the draping effects before simulating the flow with the numerical simulation LIMS.

\subsection{Modeling Shear Influenced Tow Saturation}

This numerical model will use a three-dimensional finite element mesh with one-dimensional tow elements to model the effects of fabric shear on the saturation of fiber tows. A conversion tool using Microsoft Excel macros is used to 
combine inputs from the draping simulation with a two-dimensional finite element mesh and output a final 3D model. The mesh is composed of layers of 3D elements, each layer representing an independent fabric ply in a preform. For each layer of elements in the mesh, a local shearing angle is determined from a draping simulation performed using the software FiberSIM 2009 [38].

It must be noted, however, that the draping information supplied by FiberSIM is based upon a kinematic "fishnet" model that does not account for fiber slippage. More specifically, the simplified draping does not account for the fabric's resistance to shear deformation, friction between the yarn filaments and the yarns themselves, and the resulting slippage between the fiber tows that reduces the actual local shear deformation experience by the fabric. Thus, the FiberSIM draping simulation may in fact over predict the amount of fabric shear experienced with some mold geometries. Nevertheless, FiberSIM has become a widely accepted software in the composites industry for designing and developing composite laminates and individual ply patterns. While more accurate draping models are being developed, the necessary input coefficients and computational time make them less attractive to composite designers. Also, FiberSIM model predictions are reasonably accurate as long as the fabric shearing does not reach some critical value and therefore we assume that FiberSIM is an adequate tool to use for this study. 

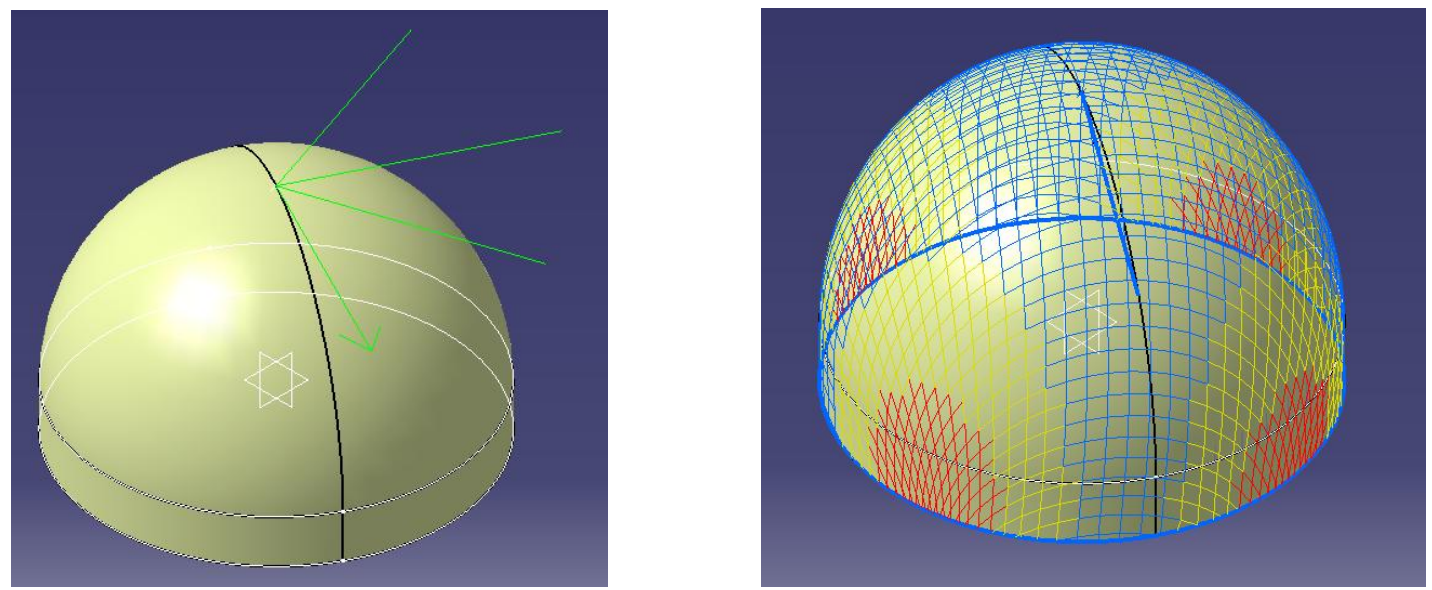

Figure 7: (a - left) Layup reference directions showing warp, weft, and $45^{\circ}$ directions and $(b$ - right) Local shear angle for the finite element draping simulation increasing from no shear (blue) to high local shear (red)

The draping simulation allows each ply in the preform and the corresponding thickness, orientation, and material properties all to be represented in the model. FiberSIM defines the warp and weft directions for each element in the mesh as well as the local shear angle. Figure 7 shows how the draping simulation is performed for fabric draped over a hemispherical tool surface. The average shearing angle of the 3D elements surrounding each base node of the 1D element is then calculated using Excel. The warp and weft directions as well as the local shear angle for each element of the draping simulation is mapped to the local coordinate system of the preform mesh created using ABAQUS [39]. This value is then used to determine how the properties of the 1D element are to be adjusted based on the corresponding material property curves (how permeability of the fiber tows change with shear angle). The 3D mesh is then created using calculated values from the two simulation input files, as well as material properties curves as shown in the flowchart in Figure 8. 


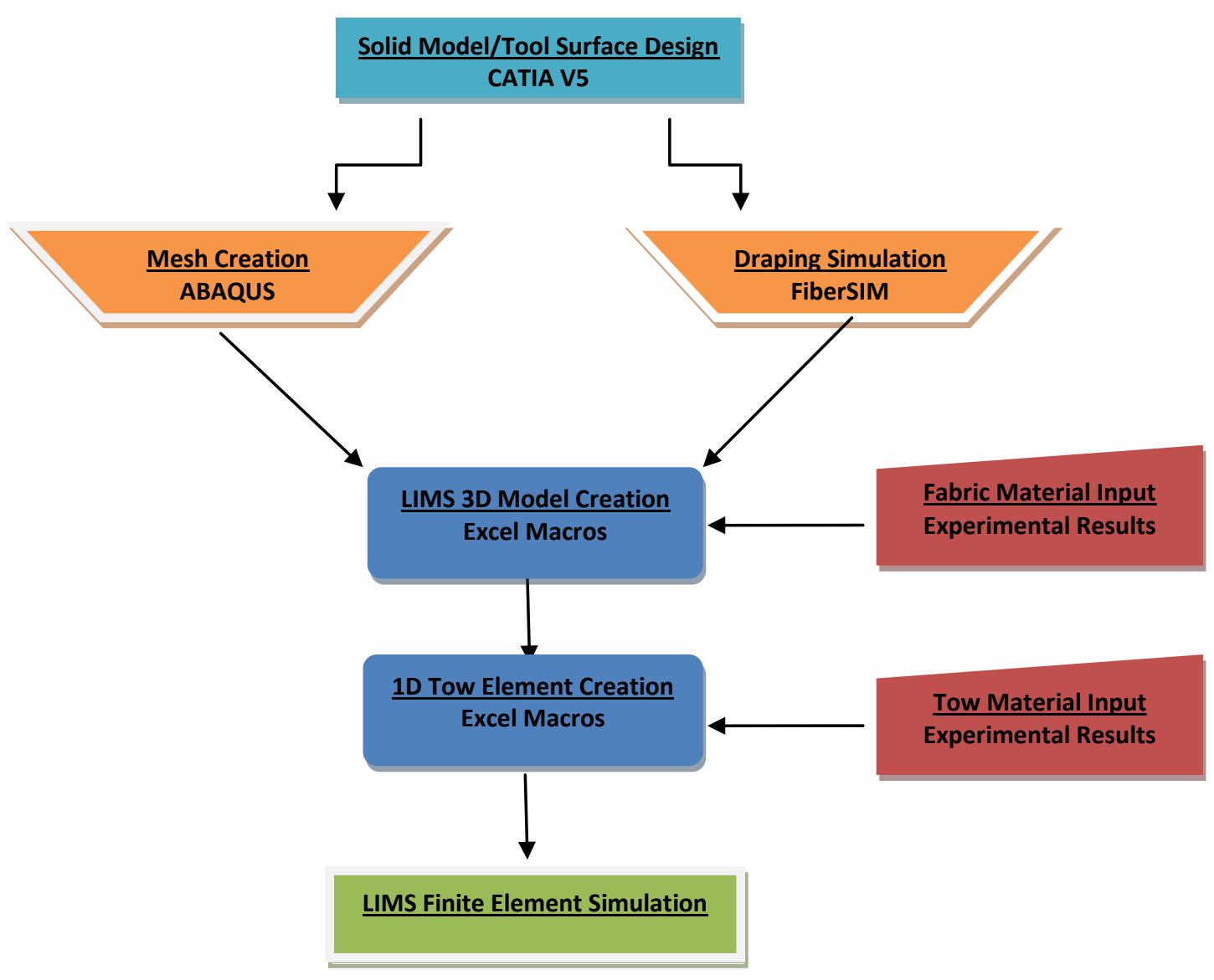

Figure 8: Flow chart delineating steps in the modeling process

Previous models used a 2D mesh with averaged preform properties in the thickness direction, giving approximated bulk permeability for each element. This 3D mesh allows for each layer to have ply specific information. With this ability, we can more accurately simulate varying ply materials and orientations. This also allows for the user to view the flow front progression and then evaluate the draping effects on a ply-by-ply basis [40]. 
Now that the initial 1D tow element properties are established, we now look at how fabric shear affects the fiber tows and adjust the model to account for these effects. As the fabric is sheared, the cross-sectional area and permeability of the tow are changed [22] due to crimp and induced tension. It is difficult, however, to correctly define and accurately model how tow geometry changes as a function of fabric shear throughout different locations within a mold. There may be both positive and negative fluctuations in tow cross-section, as well as possibly no changes at all. Another concern is that the characteristic length of the tow elements, $h$, is difficult to model due to the overlapping tows of a woven fabric. When tows overlap, the characteristic flow length doubles. Trying to combine this fact with unpredictable fluctuations in tow geometry make it extremely difficult to model the correct tow geometries, especially in a comparatively coarse finite element mesh.

For this reason, the changes in tow geometry are not modeled in the 1D element cross-section, length, or porosity. Instead, an effective permeability, $K / h^{2}$ is introduced to consolidate these changes into one parameter. This reduces the potential error in the model simulations from incorrectly modeling variations in tow geometry due to shear.

An additional concern when modeling tow saturation is that of capillary effects. When the fluid flow through the fibrous preform becomes significantly slow, capillary effects which are independent of the driving fluid pressure become important. It is difficult, however, to describe and model when this phenomena starts to come into effect, or when it is negligible. Also, the LIMS software does not currently allow one to separately model capillary effects, nor directional tow permeability. Instead, we are modeling effective tow permeability with the 
assumption of isotropic tow permeability and saturation. Again, this is a reasonable assumption due to the fact that the fiber tow effective permeabilities are characterized from empirical data. These challenges further uphold the decision to lump the varying tow parameters into one non-dimensional term that fully describes the saturation behavior of the deformed fiber tows.

For each type of fabric, material input files relating how the fiber tow permeability changes with an increase in local fabric shear angle are needed. The shear angle dependent material properties curve used in this thesis are shown in Figure 9. Note that once this curve is characterized for a fabric, this methodology can be used to explore the effects for any textile fabric.

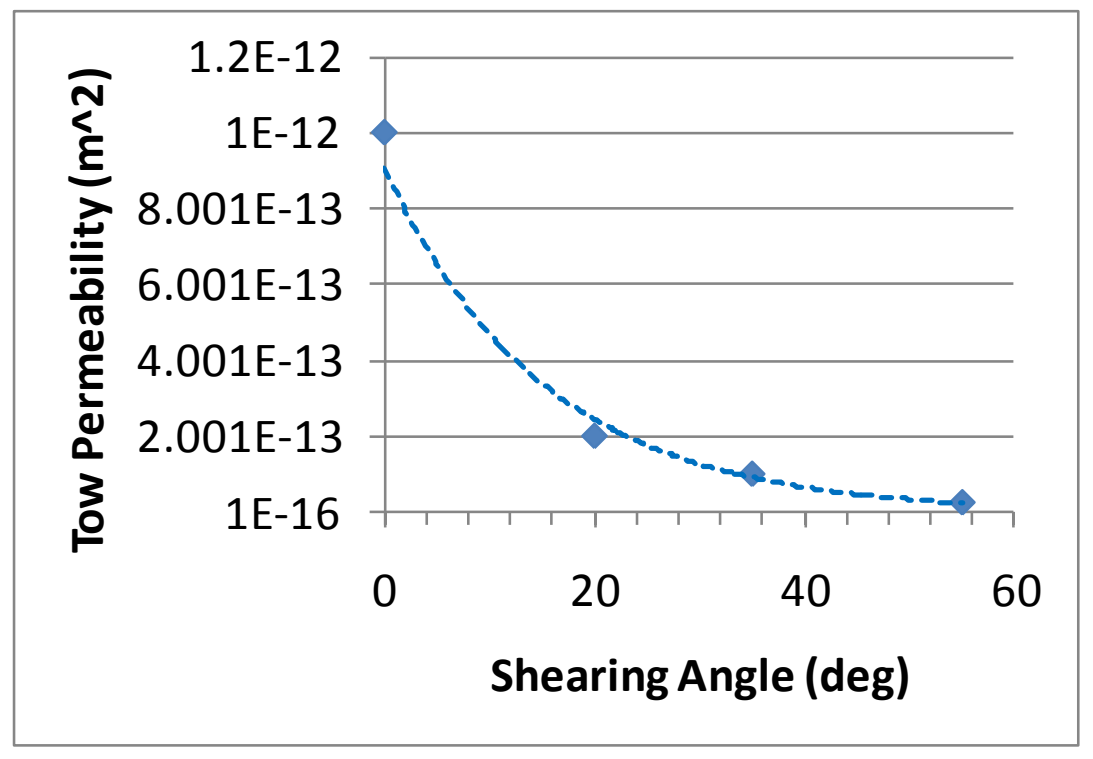

Figure 9: Shows a reduction in tow permeability with an increase in fabric shear and the corresponding exponential fit line 
The proposed three-dimensional finite element mesh accounts for how local fabric shear influences both aspects of the dual scale flow and can be used to simulate dual scale flow in liquid molding processes. Thus the mold regions subjected to high fabric shear causing the bulk and fiber tow permeability, volume fraction and fiber orientation to change will result in different flow dynamics and can impact the filling of fiber tows. This resin impregnation information in between fiber tows and into the fiber tows will also aid in mold and part design, give insight into appropriate material selection, as well as help estimate filling times. 


\section{Chapter 3}

\section{EXPERIMENTAL WORK}

\subsection{Introduction}

In order to validate the hypothesis that fabric shear does indeed influence tow permeability and saturation during LCM processes, a set of experiments were designed and conducted to confirm or refute this theory. Neacsu et. al. [37] developed an experimental procedure in which resin flows through a glass preform and fiber tow saturation can be visually observed. These experiments take advantage of the similar optical properties of glass fibers and vinyl ester resin. When the glass fibers are saturated with the vinyl ester, they become translucent. This can be seen by the wetting of a glass fiber tow in Figure 10 below. This physical phenomenon can be used to track both the bulk resin flow front, as well as the degree of tow saturation as a function of time by measuring the amount of light that is transmitted through the preform. This same approach will be used in the following experiments. 



Figure 10: Demonstration of the similar optical properties of glass fibers and vinyl ester resin. As the two are combined and the glass fiber tow wets out, it becomes translucent [41].

\subsection{Preform Permeability as a Function of Fiber Volume Fraction}

Before we start investigating the effect that fabric shear has on dual scale flow, we must first characterize the undeformed bulk fabric for comparison. The fabric of interest is a plain weave S-Glass with an areal density approximately 830

$\mathrm{g} / \mathrm{m}^{2}$. Utilizing a resin transfer molding (RTM) process, the bulk fabric permeability can be obtained by tracking the fluid flow front versus time through the preform as shown in Figure 11. 


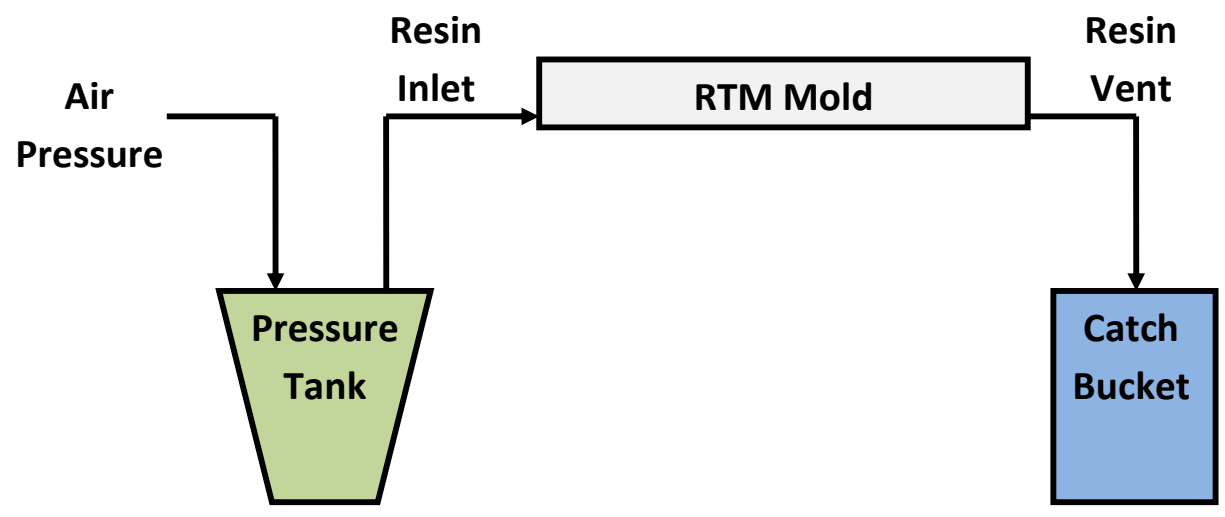

Figure 11: RTM experiment schematic

Figure 12 displays several layers of fabric stacked inside the aluminum mold cavity, and then a clear acrylic top mold enclosing the preform. The two mold halves are then clamped together to withstand the positive pressure. The fluid under pressure is introduced into a mold through a line gate as shown in Figure 13.
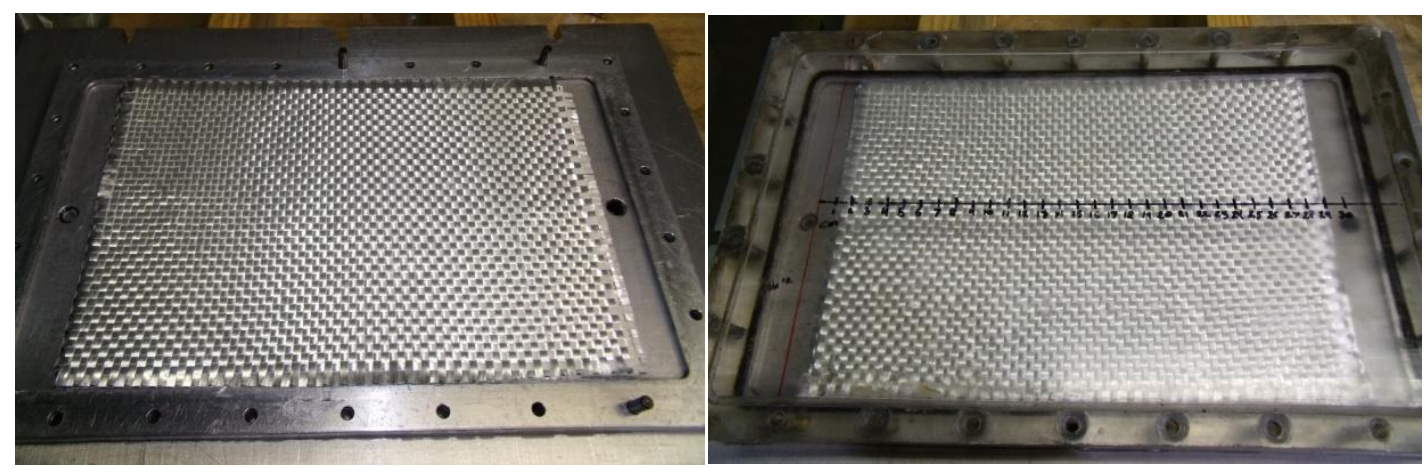

Figure 12: Closed mold RTM setup

For the following one-dimensional flow experiments, several different process parameters were varied to see their influence on bulk fabric permeability. Specifically, three different fiber volume fractions were investigated by varying the 
number of fabric plies present in the mold cavity. Since the mold cavity is of a constant thickness, the addition of more layers increases the fiber volume fraction. As was discussed earlier with the Kozeny-Carmen equation as in Equation 2, preform permeability generally changes as a function of fiber volume fraction. For this specific fabric, three different FVFs were considered $\left(v_{f}=42 \%, 54 \%\right.$, and $\left.63 \%\right)$. The process viscosity was also varied by changing the experimental fluid. Corn oil ( $\mu \sim 65$ сp) and syrup ( $\mu \sim 220 \mathrm{cp}$ ) were both used to characterize the permeability of the fabric to ensure that fluid viscosity did not have an effect on the measurements. Because the future experiments will be conducted using a VARTM process, similar pressure was used for fluid injection for these 1D RTM experiments. While the injection pressure was initially varied between 10 to 20 psi for several experiments to again ensure that it did not influence results, the experiment pressure will be defined as approximately $15 \mathrm{psi}$.

The fiber volume fraction of the preform is of primary importance in these experiments, as permeability is a strong function of the preform fiber volume fraction. In order to determine preform porosity, the following equation was used:

$$
\varphi=1-v_{f}=1-\frac{w_{f}}{\rho_{f} V}
$$

where $w_{f}$ is the total weight of the dry fibrous reinforcement, $\rho_{f}$ is the bulk fiber density, and $V$ is the volume of the mold cavity. 


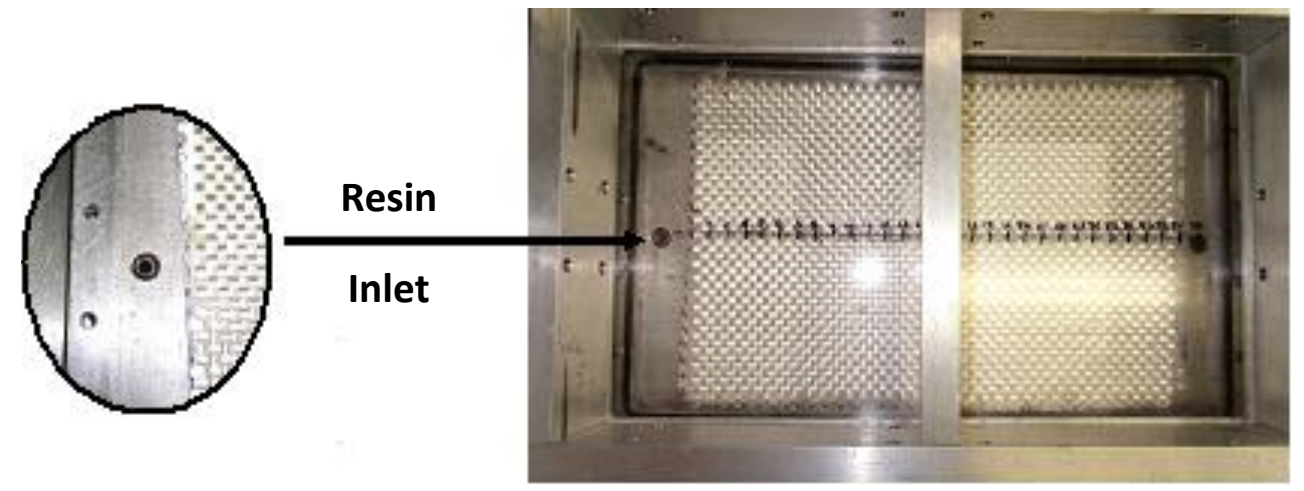

Figure 13: Resin inlet and air channel in RTM experiment setup

With the given process parameters, one-dimensional flow experiments were conducted in order to characterize the fabric permeability in the warp direction. Resin was injected, and the experiment was visually recorded with an overhead camera to capture the progression of the fluid flow front as a function of time (Figure 14).
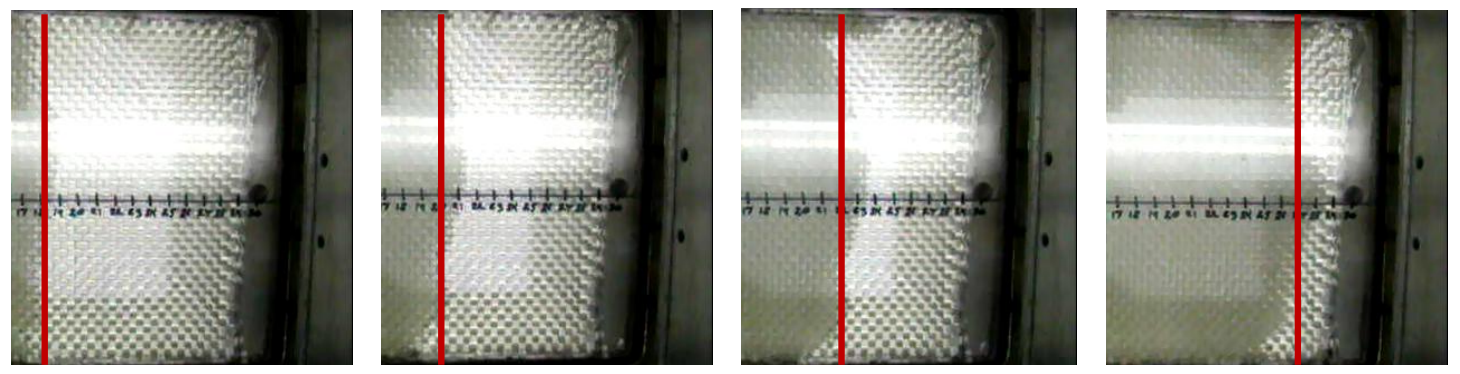

Figure 14: Tracking of fluid flow front for $1 D$ flow experiment

The permeability is calculated using the square of the flow front position length, the elapsed time, and the process parameters according to the integration and 
rearrangement of the one-dimensional Darcy's Law equation (Equation 4) for permeability:

$$
\mathrm{K}_{\mathrm{xx}}=\frac{\varphi \mu}{2 \Delta \mathrm{P}} \frac{\mathrm{L}^{2}}{\mathrm{t}}
$$

where $\varphi, \mu, \Delta \mathrm{P}$ are the experimental porosity, viscosity, and pressure drop respectively, and $L^{2}$ and $t$ are determined from tracking the resin flow front as a function of time as shown in Figure 15.

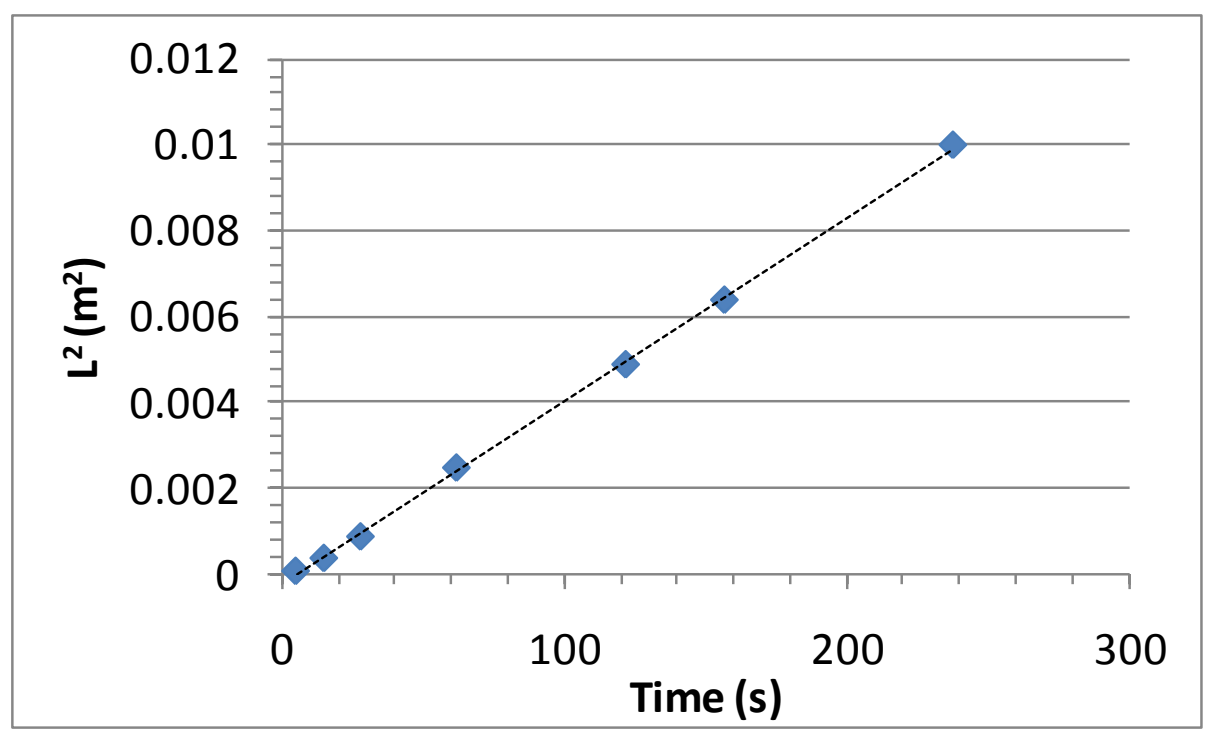

Figure 15: The slope of square of the resin flow front position as a function of time when resin is injected under constant pressure is related to the permeability of the preform

Replicates were performed to determine the average bulk permeability in the warp direction for the three fiber volume fractions of interest. The results listed in Table 1 and Figure 16, where permeability decreases along with porosity. 
Table 1: Warp permeability as a function of fiber volume fraction

\begin{tabular}{|c|c|}
\hline $\mathbf{v}_{\mathbf{f}}$ & $\mathbf{K}_{\mathbf{x x}}\left(\mathbf{m}^{\mathbf{2}}\right)$ \\
\hline 0.42 & $6.37 \mathrm{E}-10$ \\
\hline 0.54 & $1.22 \mathrm{E}-10$ \\
\hline 0.63 & $1.48 \mathrm{E}-11$ \\
\hline
\end{tabular}

The Kozeny constant described in Equation 2 was established for each measured warp permeability value. Because this constant varies for each of the experimentally measured permeabilities, the average value was used in calculating the best fit line shown by the Kozeny-Carmen line in Figure 16 below. It is this equation that is used to calculate the undeformed fabric permeability at the known experimental preform fiber volume fraction. 


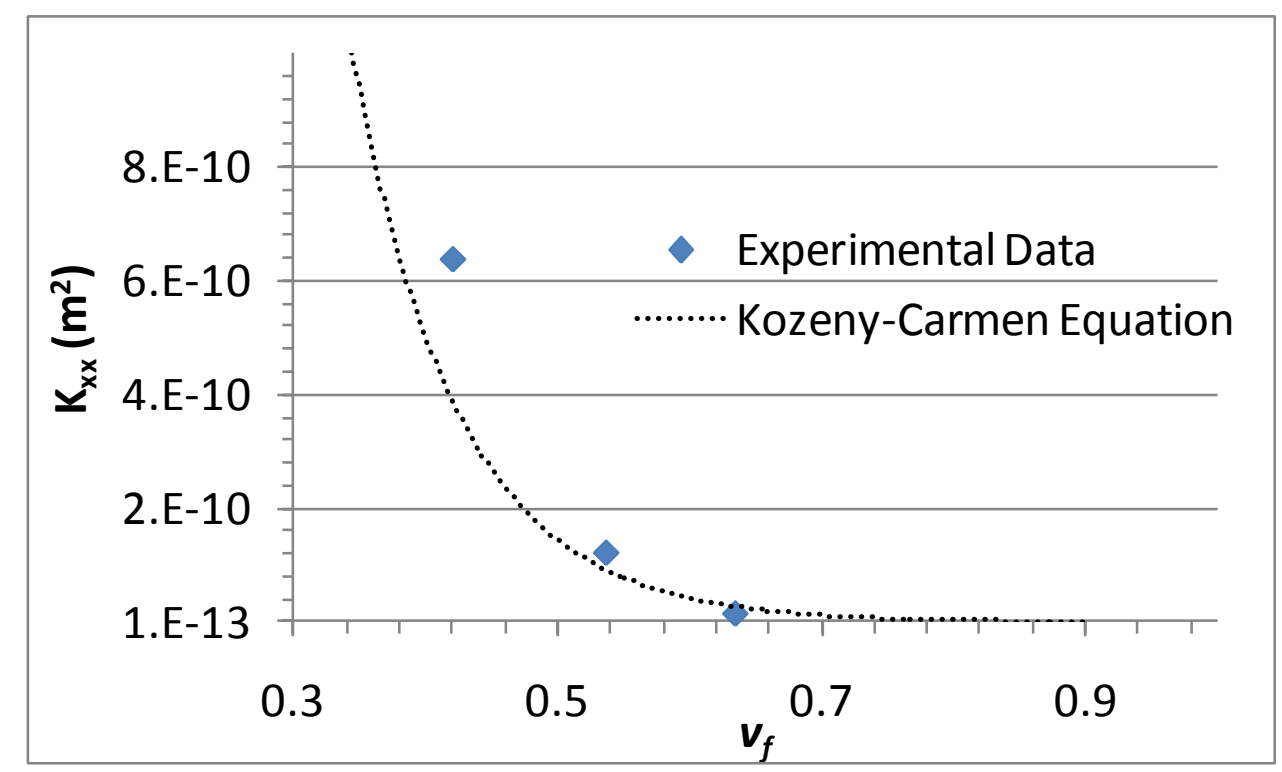

Figure 16: Average warp permeability as a function of fiber volume fraction

We now have the bulk permeability in the warp direction characterized for the fabric that will be used throughout all future experiments. This information will be useful in analyzing future data and fully characterizing the fabric and tow permeability as a function of shear angle, which is the goal of the next set of experiments.

\subsection{Fiber Tow Saturation and Permeability as a Function of Shear}

In order to determine how fabric shear affects the processing characteristics of woven preforms, a new set of experiments must be designed and conducted in order to capture these effects. For the purpose of these experiments, the following experimental setup was used. The glass preform is placed between the transparent acrylic tool and the caul plate. In a dark enclosure beneath the preform and tool plate, a video camera records the amount of light that is admitted through the 
wetted preform. A light source is situated above the preform to provide the correct amount of dispersed light for the camera to accurately pick up the varying level of fiber tow saturation. A simplified one dimensional flow experiment conducted by [37] is shown below in Figure 17.
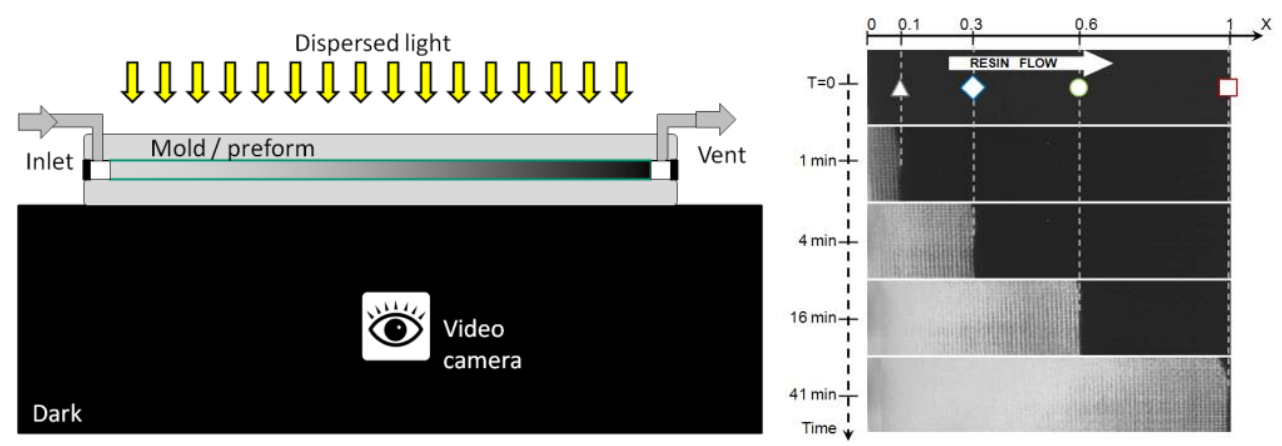

Figure 17: (a - left) A diagram of the experimental setup used by [37] to capture tow saturation data for the one-dimensional flow experiment shown in ( $b$ right) [42]

The experiments conducted in this thesis are a modified version of previously conducted experiments reported in [37]. In order to investigate the effect of fabric shear on fiber tow saturation, a shearing fixture is introduced into the setup to vary the induced shear angle on a square preform. The shearing fixture shown in Figure 18 was machined out of aluminum to hold 20 inch square preforms of various thicknesses, with possible shear angles ranging up to 40 degrees. It is assumed that the fabric will slip after the locking angle which is close to 40 degrees for this fabric. The edges of the preform are clamped between textured rubber strips to firmly grasp the fabric and eliminate slippage. The sheared fabric and fixture are then centered over a 
12 inch square acrylic plate that represents the region of interest. This was done to ignore any edge effects that the clamped fabric may introduce and influence resin flow.

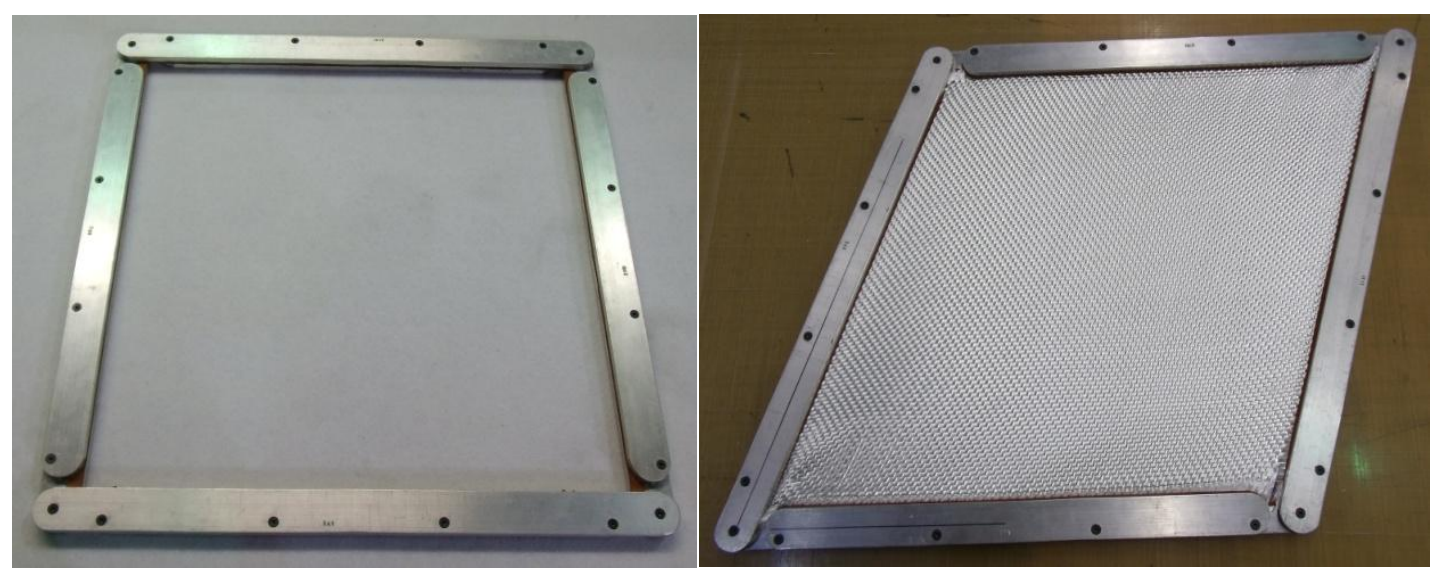

Figure 18: (a-left) The aluminum shearing fixture used throughout the designed experiment and (b) four layers of Owens Corning $24.5 \mathrm{oz}$. plain weave ShieldStrand-S fabric sheared to $20^{\circ}$

As stated previously, the fabric used is a plain weave S-Glass with 5.5 ends and 6.1 picks per inch manufactured by Owens Corning (24.5 oz. ShieldStrand-S $\left(830 \mathrm{~g} / \mathrm{m}^{2}\right)$ with a vinyl ester compatible sizing). This unbalanced fabric can explain why a flow ellipse is witnessed versus a perfect circle. As the fabric is sheared, the flow ellipse rotates, as is seen in [20]. The preform comprised of four plies of fabric, as it yielded the best contrast between the macro flow front and the fully saturated region, making it clear to see the partially saturated tows. In order to create a uniform flow profile through the thickness of the preform, a $3 / 8$ inch diameter hole was punched in the center of the fabric at the location of the injection site. 
The resin used was Hetron 980/35 vinyl ester with a 2 hour gel time. The long gel time eliminates significant fluctuations in resin viscosity, allowing one to confidently assume that resin viscosity is a constant experimental parameter during the characterization of the fabric. The resin viscosity was then modified by the addition of styrene monomer to increase flow velocity and eliminate any capillary effects that may arise during creeping flow.
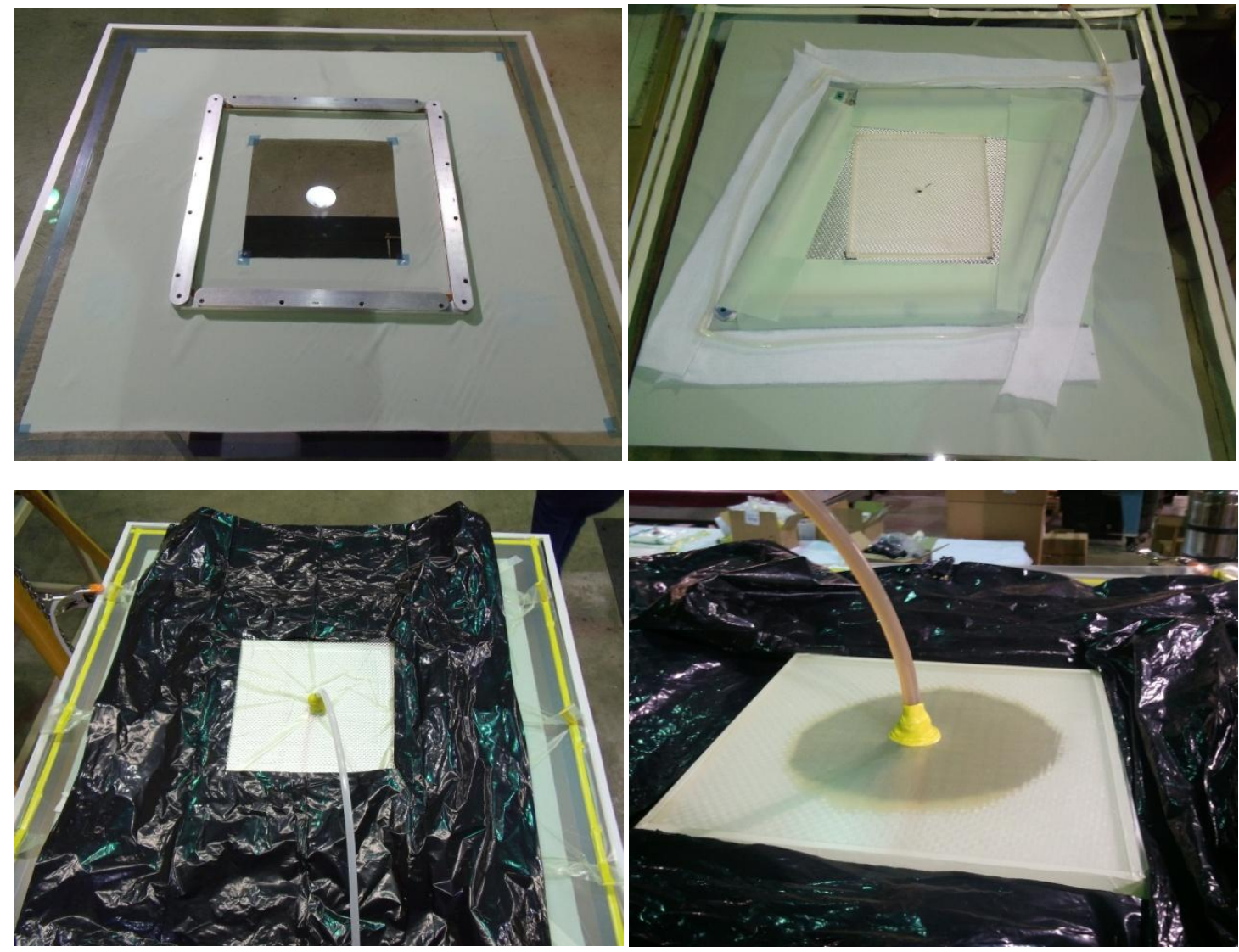

Figure 19: (a - top left) The shearing fixture is centered over a clear 12 inch square that represents the region of interest where video data will be collected, $(b$ - top right) the sheared fabric and fixture laid up on the tool surface in a VARTM setup, (c-bottom left) the vacuum bagged experimental VARTM setup, and ( $d$-bottom right) a radial flow experiment underway. 
A radial flow experiment was then conducted using the vacuum assisted resin transfer molding (VARTM) process. Video data was collected for the entire experiment, capturing the progression of the flow ellipse until it reaches the edges of the 12 inch region of interest. Still images are extracted from the video at set time intervals. This image series is the raw input data loaded into an image processing code that quantifies the flow characteristics of the experiment.
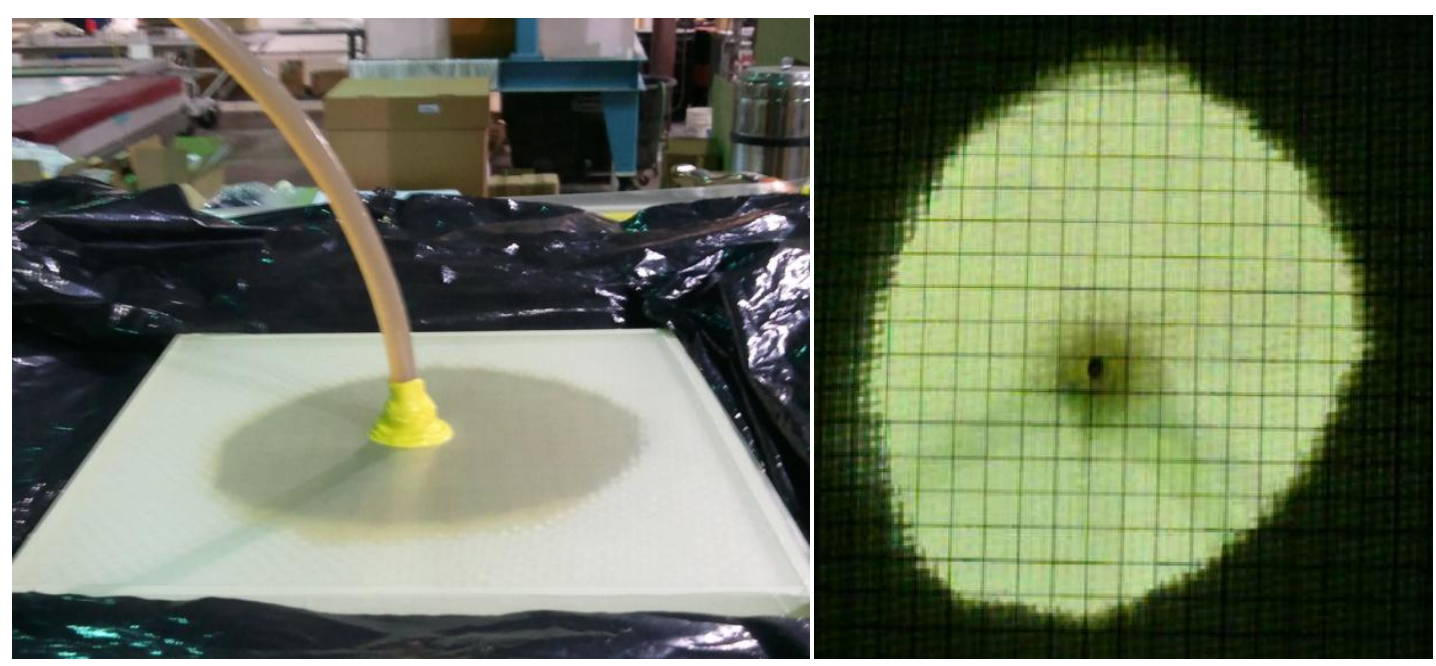

Figure 20: $(a$ - left) Radial flow experiment from a top and ( $b$ - right) bottom view.

\subsection{Image Analysis}

An image processing code was written using the MATLAB Image Processing Toolbox software to automatically filter and process all images in the experiment time series. The program was written to be robust and versatile enough to 
be accurate for any experiment with the presence of variable light intensity and therefore image contrast. The program prompts the user for a directory containing all of the images in the experiment time series and allows them to select and crop the region of interest. After regions of interest and the location of the experimental images are selected, the program automatically performs a set of functions to process and analyze each image, until all images in the series have been cycled through. The different image analysis processes will now be described.

The first step when dealing with these types of images is to crop and convert the RGB image to an intensity image with single precision. This results in a loss of color data for each image, but for the purpose of these experiments, this is irrelevant. This conversion can be seen below in Figure 21. The benefit of this operation is that each image is now treated as an $m$ by $n$ array in which each matrix cell value represents the corresponding pixel intensity value. This allows us to easily perform the necessary smoothing and transformation functions.

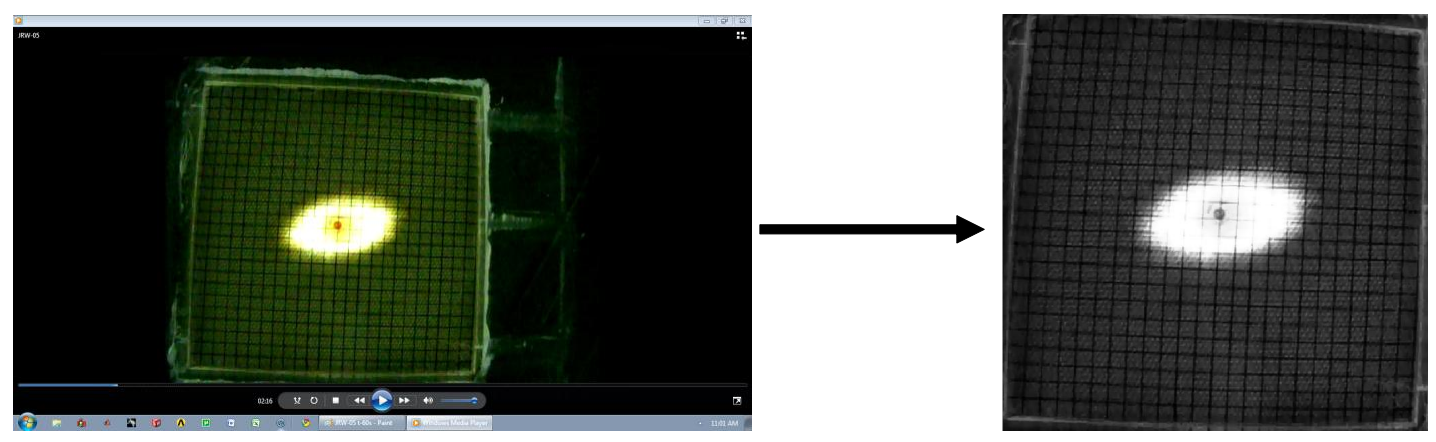

Figure 21: Original raw experimental image cropped and converted to an intensity image for processing. 
Next, we had to correct for an image intensity drop with the progression of time. As each experiment progressed, more light was allowed to transmit through the wetted preform into the dark enclosure below. This caused the camera recording the experiment to automatically correct for the proper light exposure resulting in the continual darkening of the background of each image in the time series. This can again be seen in Figure 22.
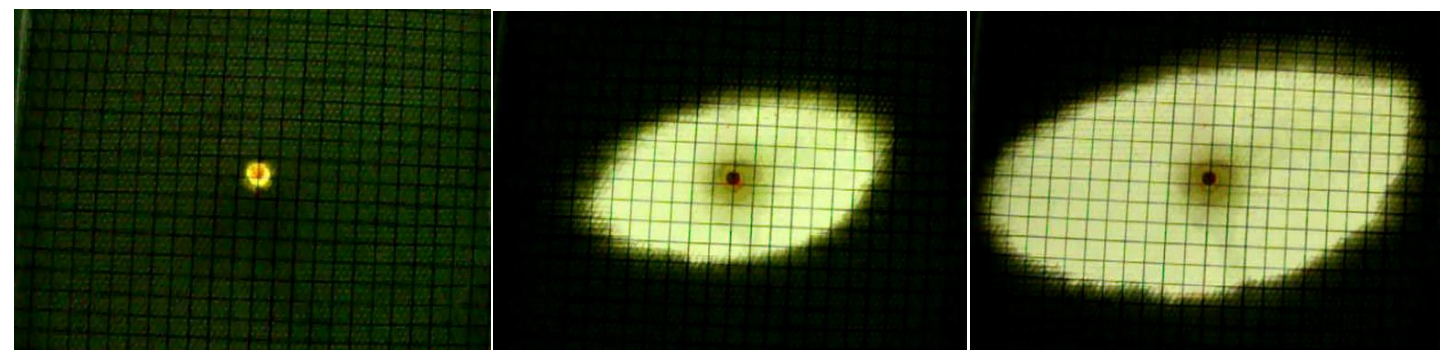

Notice darkening of image

Figure 22: Image background continually grows darker due to increased light exposure as time progresses and more glass fibers are saturated.

To correct for this image intensity drop, the user is prompted to select a region far away from the resin flow front to be used for intensity correction. For these experiments, a small rectangular region in one of the far four corners was selected for this purpose. For each image in the time series, the mean intensity of all of the pixels in this region was calculated. This mean integer value is then subtracted from each pixel for the experimental image. For the user selected region, the minimum intensity value is calculated and subtracted from the experimental image again. The results of these operations can be seen in Figure 23. This pixel adjustment operation normalized 
all images in the series to relatively the same base intensity value for the non-saturated regions far away from the resin flow.

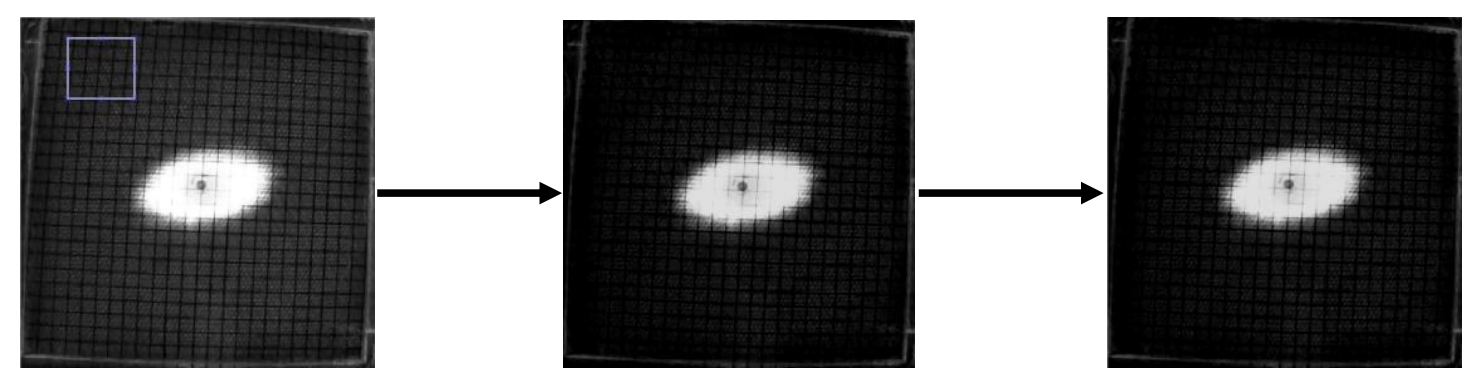

Figure 23: Correction of image intensity drop for each image by subtracting a mean base intensity value.

Now that all images in the experimental time series have been normalized, we can look into analyzing the resin flow and tow saturation for each image. In order to perform the following operations, another image transformation must be conducted. Each image is converted to a logical, or binary image where instead of each matrix cell or pixel having an intensity value between 0 and 1 , each pixel is transformed to being "on" or "off." Here, the "on" pixels are described by a value of 1 or the color white, and the "off" pixels are 0 and black, respectively.

Different threshold values were used to extract both the macro flow front and fully saturated tow regions for each time step. To obtain the appropriate threshold values for each case, we look to the image intensity histogram shown below in Figure 24. The threshold values are declared as a percentage of the maximum intensity for the image. The percentages used are adjusted for each experiment in order to ensure 
that the correct regions are selected, but the specific values used are $10 \%-25 \%$ of the max intensity to detect the macro unsaturated flow, and $65 \%-80 \%$ to isolate the saturated flow. While there is some inherent error in this procedure, the threshold level is varied until the maximum (or minimum) value that yields clean, logical data, resulting in the fairly accurate detection of the resin flow front profiles. This results in the detection of the maximum difference between the saturated and unsaturated flow regions, minimizing the error (on the order of 10\%) between the measured and actual difference between the bulk and saturated flows.

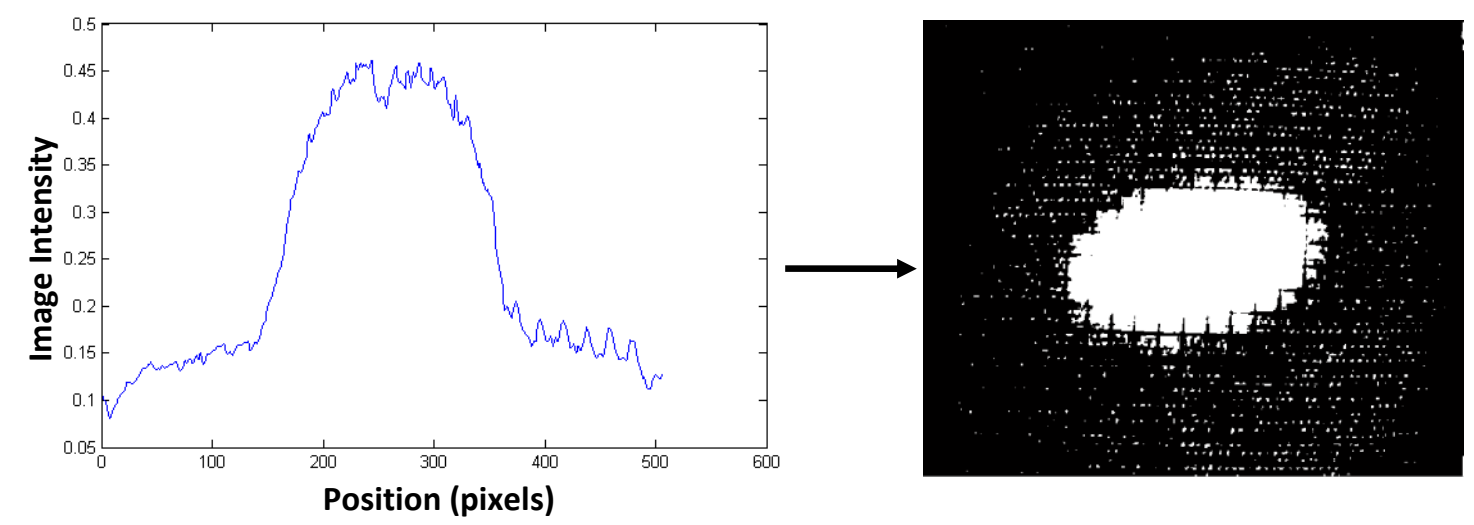

Figure 24: (a - left) Intensity histogram displaying the image's average column vector intensity that is used to calculate the thresholding values to detect flow patterns such as the one shown in $(b-$ right $)$.

The image processing program now creates two new separate logical images representing the two flow patterns of interest: the saturated and unsaturated flow ellipses. 
The resulting binary images have accurately detected the two flow profiles of interest, but with a certain level of noise outside of those regions from the associated images represented by the scattered white pixels that were above the threshold value. Several smoothing operations were conducted to isolate the region of interest without losing significant data. First, an iterative 9 block moving average was applied to each image. The number of iterations was adjusted to balance resolution and calculation time, but typically 100 iterations was more than sufficient to properly smooth the images to remove the majority of the white noise. The second very simple operation removes all objects from the binary images under a designated area (1000 square pixels).
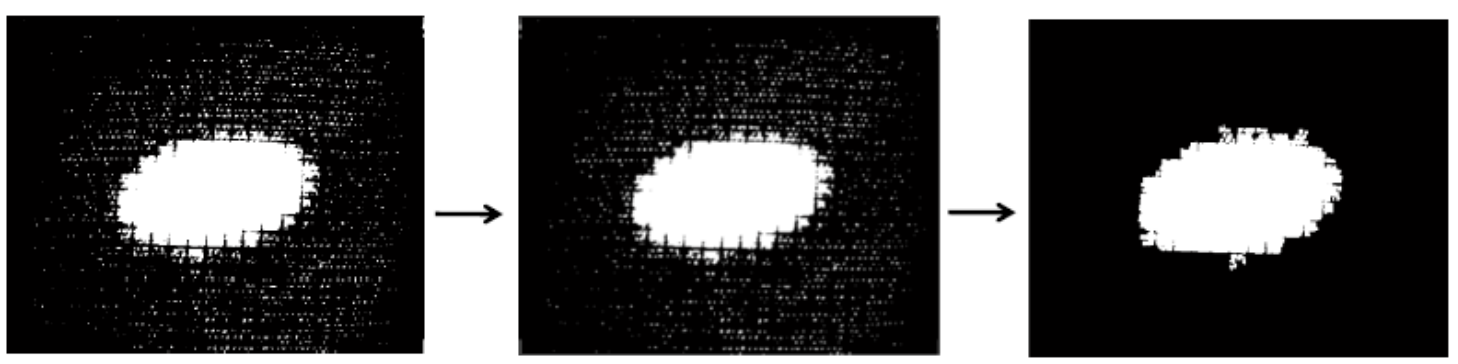

Figure 25: (a - right) Application of the 9-block averaging smoothing function, $(b$ middle) the removal of all objects under 1000 square pixels, and (c) the resulting clean binary image.

For each time step, the program writes an image file for the cropped raw image, the unsaturated and saturated logical images, as well as the difference between the two representing the partially saturated region. 


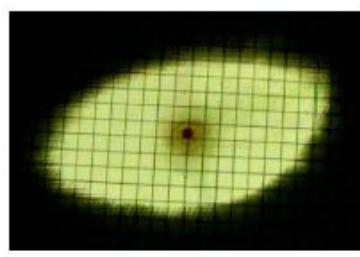

Raw Image

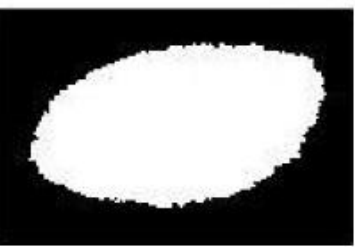

Fully Saturated

Region



Unsaturated Flow

Front

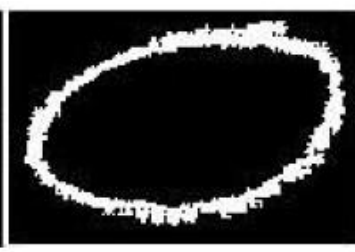

Partially Saturated Region

Figure 26: The four different image outputs from the image processing code for visual inspection and comparison of the results.

These images, mainly the two binary images representing the saturated and unsaturated flow, will be the basis for the further analytical work performed by the image processing code.

Once the MATLAB program has successfully identified the saturated and unsaturated flow ellipses for each time step of the experiment, one can quantitatively analyze the resin flow. The MATLAB function regionprops can be called to obtain information about objects in a logical image measured in pixels. Specifically, we are interested in the ellipse centroid coordinates, major and minor-axis lengths, orientation and eccentricity for both image cases (saturated and unsaturated flow). As the program cycles through each image in the time series, it records all of the listed measurement data for each time step in a detailed Microsoft Excel spreadsheet. This spreadsheet is then written to the experimental image directory for further analysis.

The major and minor-axis lengths versus time are of primary importance to the experimental measurements, but the other information allows us to fit an ellipse to the logical image via the parametric equation:

$$
X(t)=x_{c}+a \cos t \cos \varphi-b \sin t \sin \varphi
$$




$$
Y(t)=y_{c}+a \cos t \sin \varphi+b \sin t \cos \varphi
$$

where $a$ and $b$ are the semi-major and semi-minor axes respectively, $\varphi$ is the orientation angle of the ellipse, with the parameter $t$ varying from 0 to 2 .

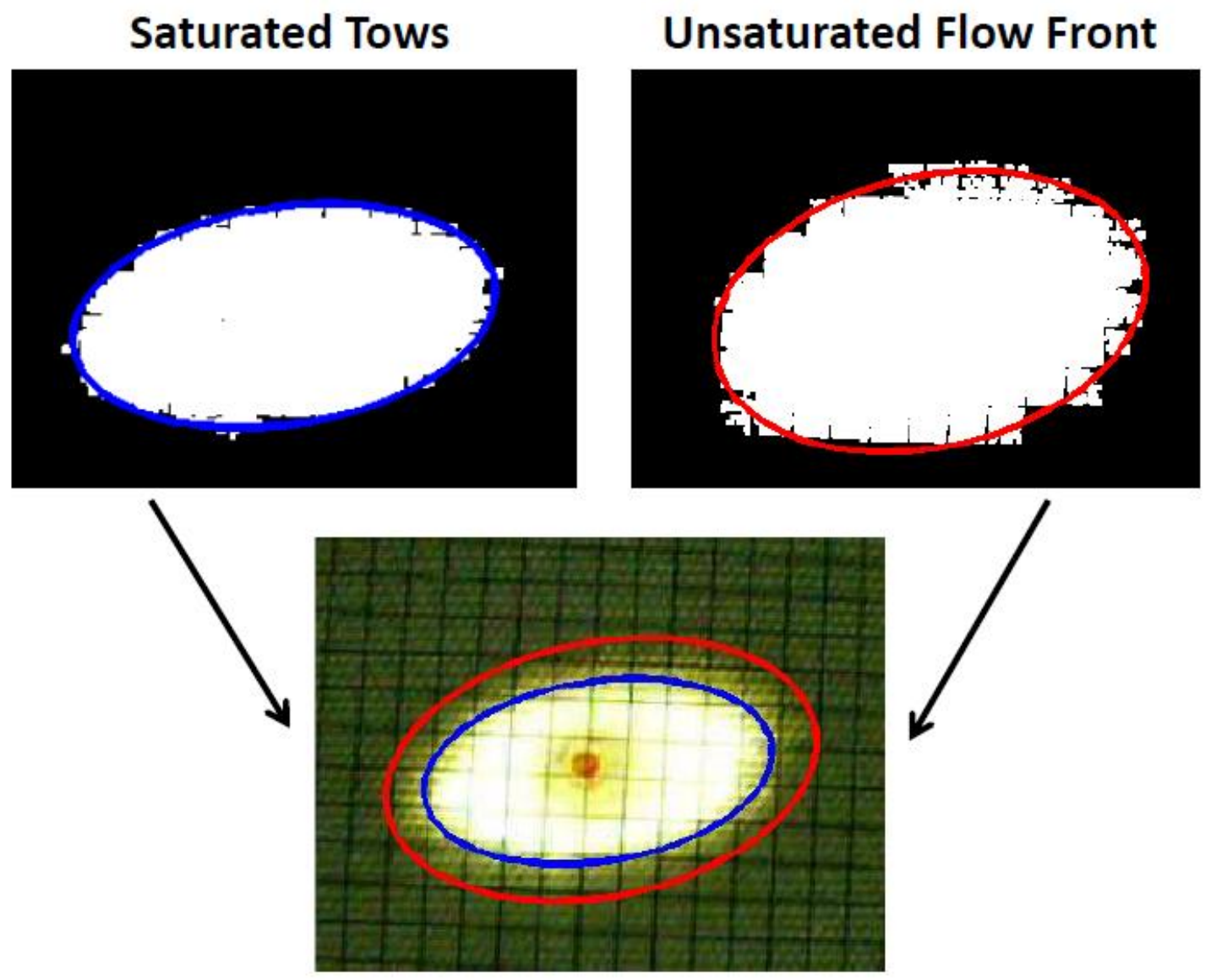

Figure 27: Best fit ellipses of logical images for both the saturated and macro flow front regions.

With the measured ellipse properties that were obtained as inputs into the parametric equation, we can now fit an ellipse to the binary images to visually confirm 
that the program is measuring the correct ellipse values. This can be performed by overlaying the parametric fit equations on the binary images, as well as the raw input experiment images as is seen in Figure 27. These methods were used for a select few images throughout an experiment to confirm that the results are accurate, and that the image processing program is sufficiently robust to work throughout the time series for any radial flow experiment. We were able to confirm that the measurements obtained by the code are correct, and that the resulting fit ellipses accurately encompass the fully saturated region (represented by the blue line in Figure 27) and the unsaturated macro flow front (red line).

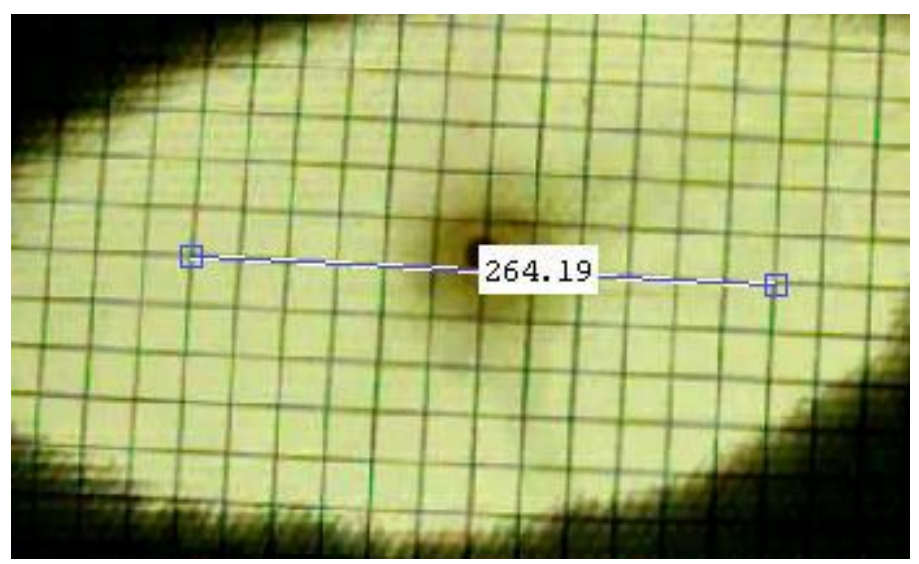

Figure 28: Determination of image scale

The only missing information needed to analyze the experimental flow data is the image scale. This is obtained by a simple MATLAB function that outputs the length of an adjustable line. When this line is compared to the grid with constant known spacing shown in each image, we can then easily find the image scale (pixels 
per inch). This is shown in Figure 28 where it can be calculated that the scale for this particular experiment is 44.03 pixels per inch (264.19 pixels divided by the known distance of 6 inches). This image processing tool has now been automated and validated and is ready to be applied to the designed experiment to fully characterize technical fabrics for a range of shear angles.

\subsection{Resin Injection Experiments with Fabric Shear}

The goal of the designed experiment was to conduct trials at shear angles of $0^{\circ}, 10^{\circ}, 20^{\circ}, 30^{\circ}$, and $35^{\circ}$ for this Owens Corning glass fabric with four replicates performed for each case. With the need to conduct twenty or more time consuming experiments, two tests were run as initial probes to see if the expected physical relationships were evident during the designed experiment. The extreme values of $0^{\circ}$ and $30^{\circ}$ fabric shear were investigated to see if there was a difference in flow characteristics between the two with all other experimental variables held constant. As can be seen in Figure 29, there is a significant difference between the two cases. There is a much larger lag between the macro flow front and the saturated tow region for the case of $30^{\circ}$ shear as expected. These two experiments confirmed that it may be possible to quantify this difference for various shear angles for the selected fabric. 

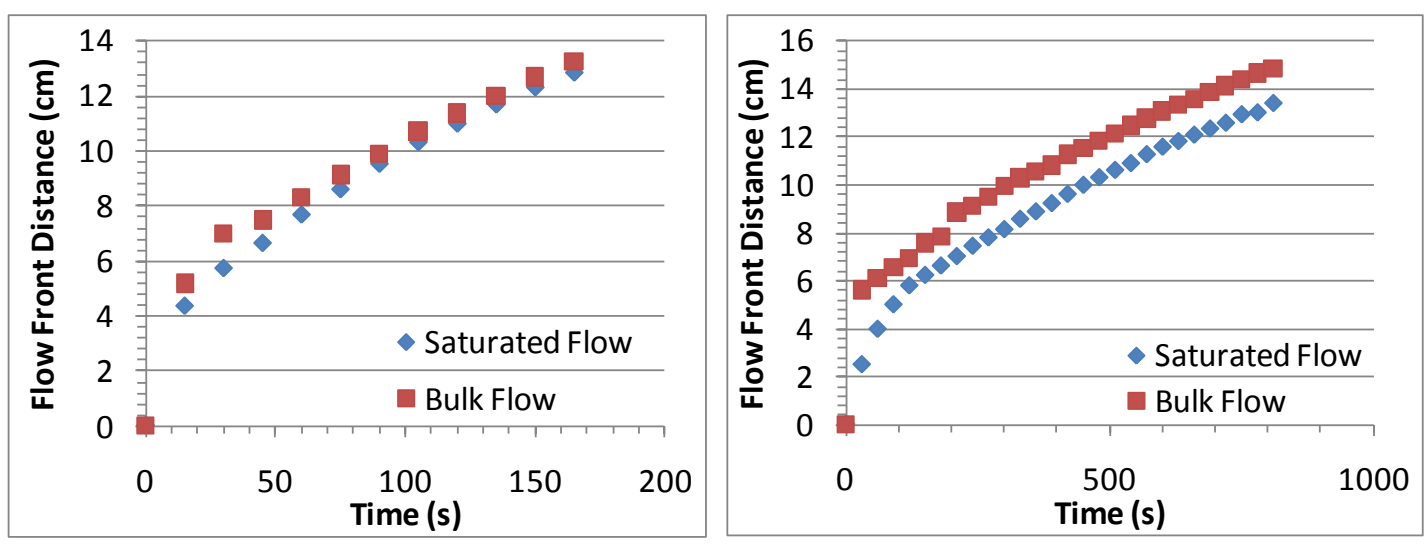

Figure 29: Flow front position vs. time for ( $a$ - left) the undeformed fabric and $(b-$ right) $30^{\circ}$ shear. There is a visually noticeable contrast of the gap between the macro flow front and the saturated fiber region.

Resin viscosity is also an experiment variable of primary importance. To study the effect that this has on the current experiment design, the vinyl ester resin viscosity was adjusted by the addition of a stabilized styrene monomer. Three different viscosities were tried for the cases of $30^{\circ}$ fabric shear and the undeformed fabric $\left(0^{\circ}\right.$ shear) and shown in Table 2.

\section{Table 2: Experimental viscosity range}

\begin{tabular}{|l|c|c|}
\hline Viscosity & Range (cp) & \% Styrene \\
\hline Low & $50-100$ & $30 \%$ \\
\hline Medium & $150-250$ & $10 \%$ \\
\hline High & $400-500$ & $0 \%$ \\
\hline
\end{tabular}




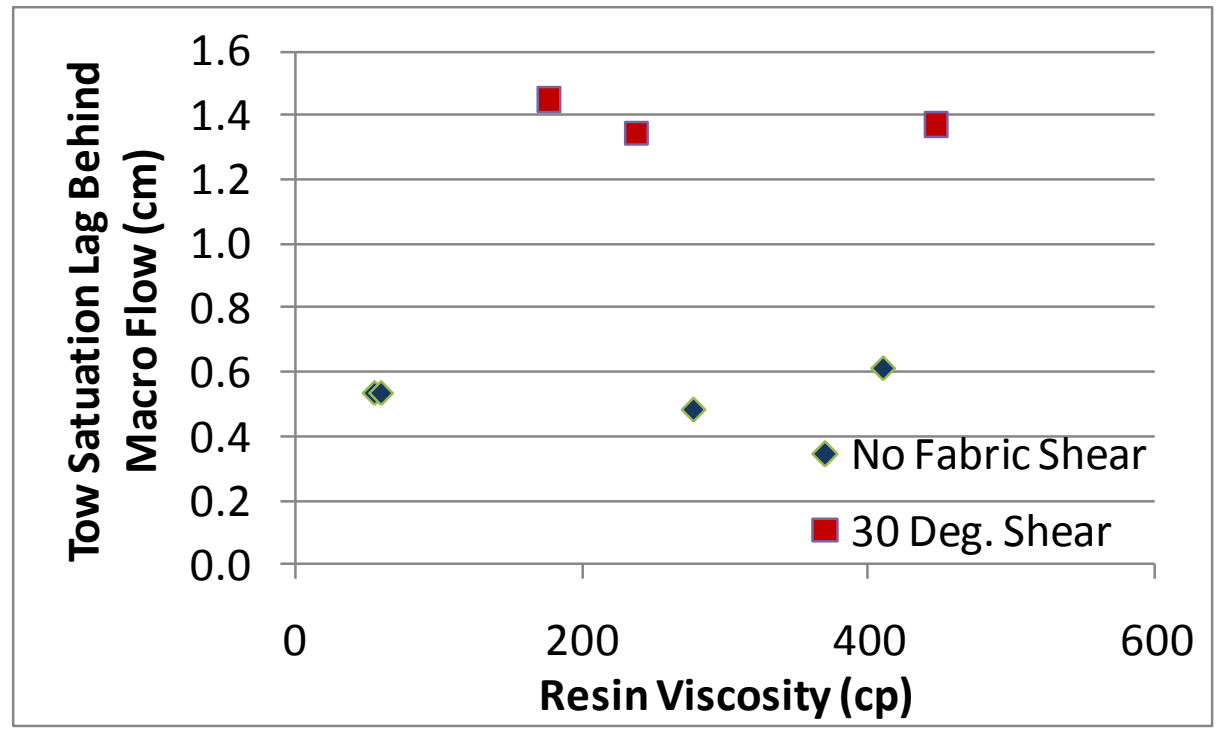

Figure 30: Resin viscosity does not appear to have an effect on the tow saturation lag experienced during the experimental trials.

While the flow speeds up significantly with the addition of styrene, the resin viscosity does not appear to have an impact on the resulting tow saturation lag behind the macro flow front as can be seen by Figure 30. This agrees with the physics of the governing equations which are linear in terms of viscosity. An increase in flow velocity between the fiber bundles is accompanied by an equal increase in flow velocity into the fiber tows. This also indicates that the flow is sufficiently fast that capillary effects are not a contributing factor, which would be detrimental to our results, producing an added variable or noise to the data.

Since resin viscosity does not seem to influence the gap between saturated and macroscopic flow front, the resin with $10 \%$ styrene by weight was chosen for all the experiments. This resulted in resin flow rate which eliminated the cause for concern of the presence of capillary effects which may contaminate the data and it also 
produced more uniform flow profiles when compared to the lower viscosity trials (Figure 31).
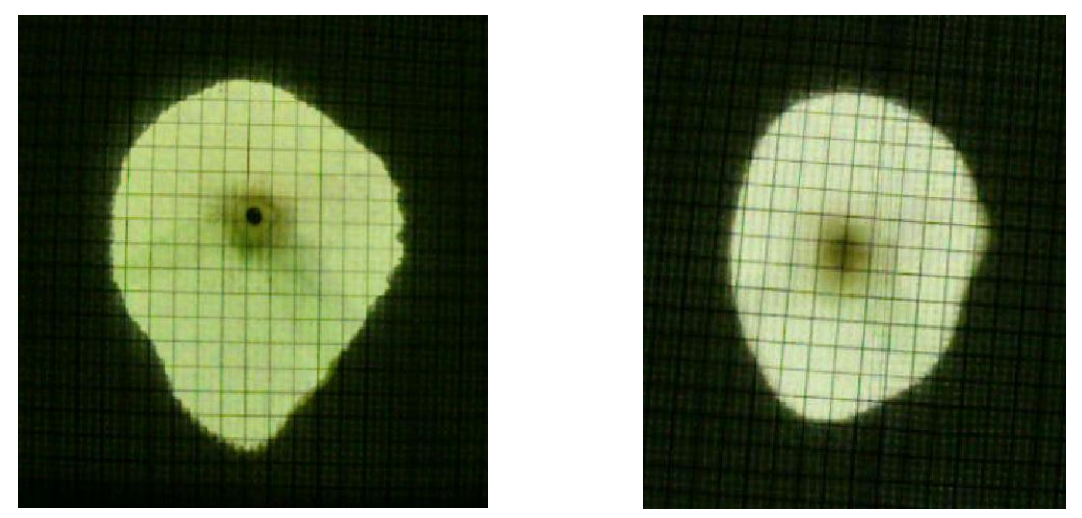

Figure 31: Difference in flow profiles for low (left) and medium (right) viscosity trials

The remainder of the experimental trials were conducted with the constant reduced viscosity, vacuum pressure, with four replicates each. One interesting observation occurred during the first trial of the radial flow experiment for fabric shear of $35^{\circ}$. As can be seen by Figure 32, there appears to be superposition of several differently oriented elliptical flow patterns progressing throughout the experiment. It was determined that this phenomenon was caused by interply slippage during shearing. While all of the fabric plies were clamped tightly in the shearing fixture, one or several of the middle plies were not gripped well by the textured rubber strips and this allowed them to oppose the full shearing of the fabric. This problem was eliminated by fusing the edges of the individual plies of the preform together with the same vinyl ester resin used during the experiment. This ensured that there would be 
no slippage between plies during fabric shearing. This method of correction was successful, and was applied to the cases of $30^{\circ}$ shear as well to be confident that the same event did not contaminate the data for that experiment as well. This additional step was unnecessary for experiments with lower fabric shear, and it would have been obvious upon visual inspection if the same interlayer ply slippage was present.
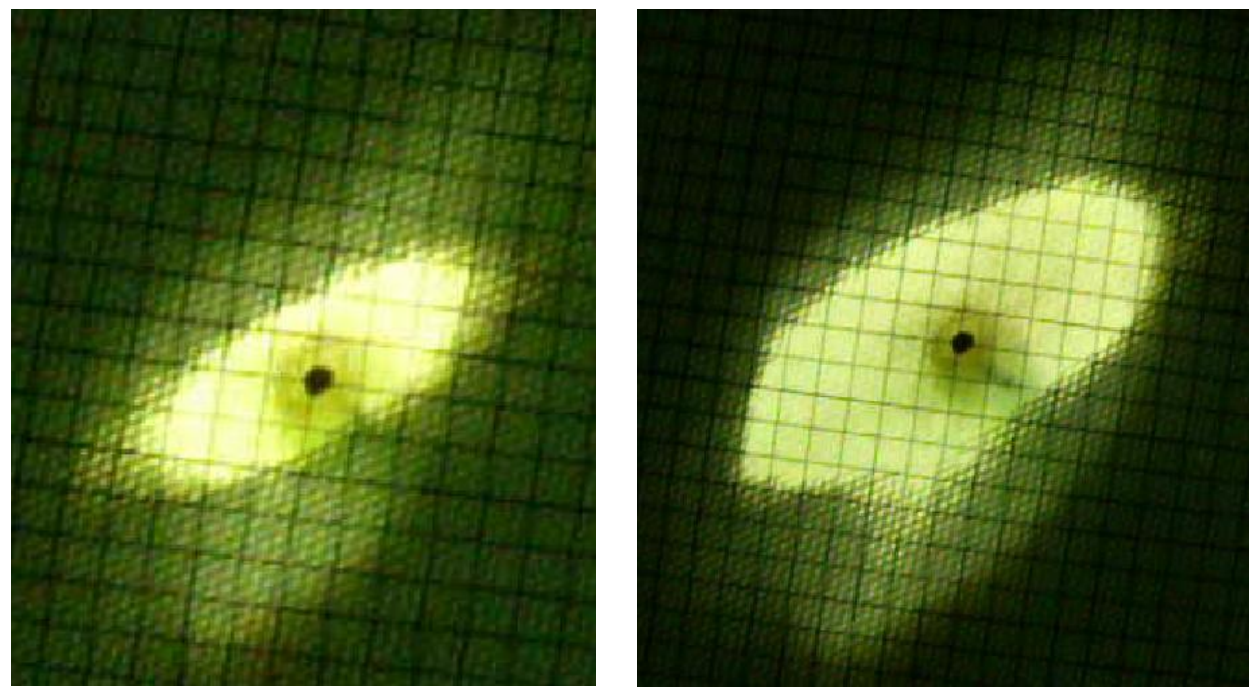

Figure 32: Multiple flow ellipses seen due to interlayer ply slippage

The experimental images were then filtered and processed by the MATLAB code discussed previously, with the appropriate threshold values used for each experiment. The saturated and unsaturated flow fronts are tracked and measured in both of the principal directions via the MS Excel table exported by the image processing code. Figure 29 gives a good representation of how the two flow fronts progress versus time for each experiment. The clear quadratic relationship represents 
initial entrance flow effects with an increasingly linear flow front position behavior as time progresses.

In addition to tracking the major and minor-axis flow front lengths, the orientation of the flow ellipse as a function of fabric shear was also recorded. The principal flow axis direction rotates from the normal warp, or $\boldsymbol{x}$-direction to the new $\boldsymbol{x}_{\boldsymbol{I}}$ direction as the fabric is sheared. This angle, $\beta$, can be seen below in Figure 33.

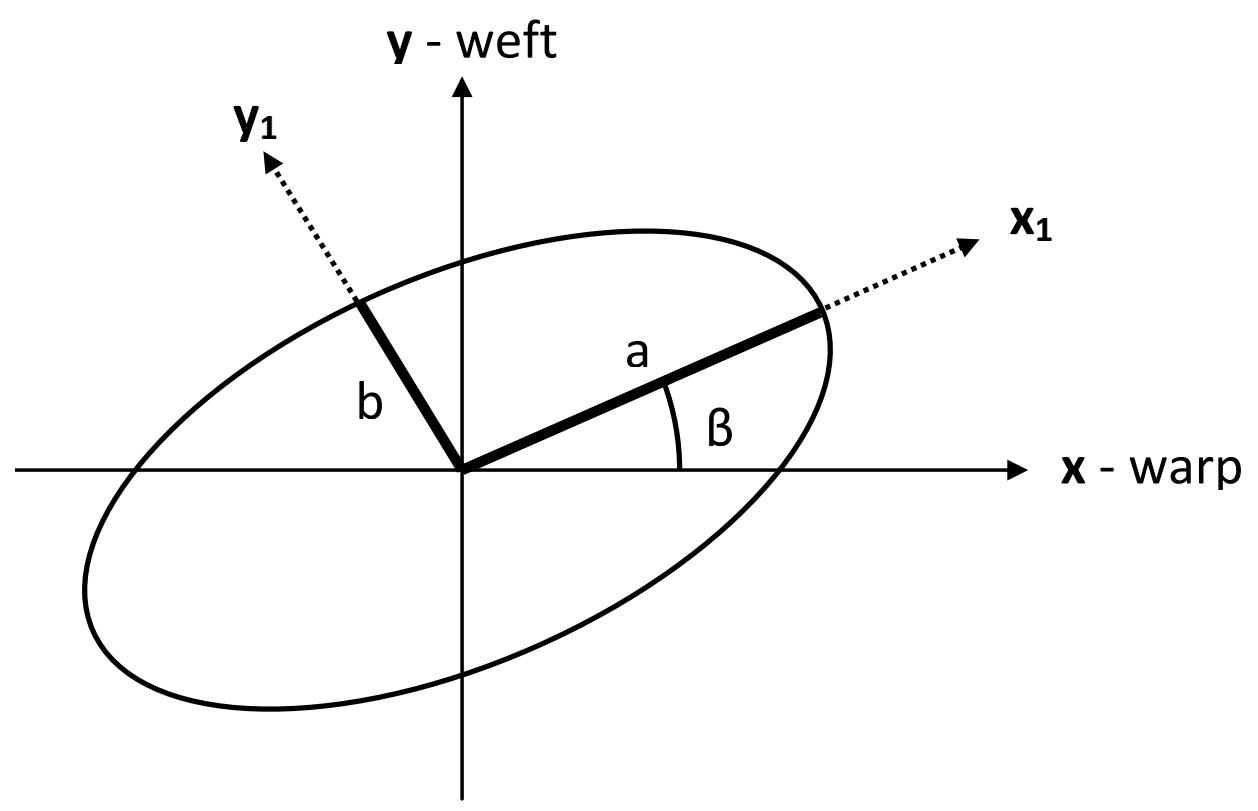

Figure 33: Rotation of the elliptical flow pattern 
The results from this study can be seen below in Table 3 and Figure 34, the rotation angle $\beta$ increasing with fabric shear as expected. This information is a necessary input for the mesh generation program and numerical model described in the previous chapter in order to describe the rotation of the principal permeabilities.

Table 3: $\quad$ Measured rotation of principal flow direction as a function of fabric shear angle

\begin{tabular}{|l|c|c|c|c|c|}
\hline \multicolumn{7}{|c|}{ Principal Axis Roatation Angle $\boldsymbol{\beta}$} \\
\hline & $0^{\circ}$ & $10^{\circ}$ & $20^{\circ}$ & $30^{\circ}$ & $35^{\circ}$ \\
\hline & 1.1 & 17.0 & 24.8 & 29.6 & 34.2 \\
\hline Average ( ${ }^{\circ}$ ) & 0.9 & 0.4 & 2.7 & 0.7 & 3.9 \\
\hline Standard Deviation &
\end{tabular}

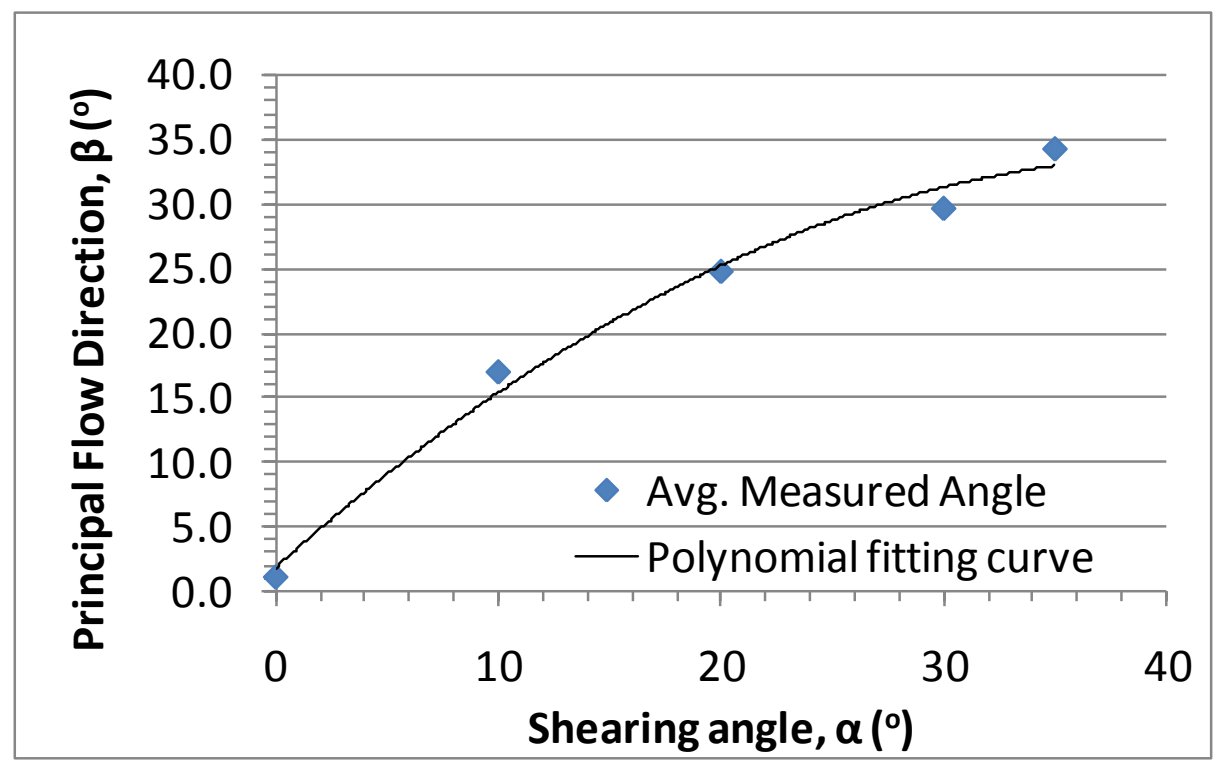

Figure 34: Rotation of principal flow direction by angle $\beta$ 
In order to analyze the shear dependent results from one experiment to another, the difference between the faster macro flow front and slower moving saturated tow area $\Delta L$ was determined and declared as the tow saturation lag (Table 4).

Table 4: $\quad$ Table calculating tow saturation lag

\begin{tabular}{|c|c|c|c|}
\hline Time (s) & $\Delta_{\mathbf{L}_{1}(\mathbf{c m})}$ & $\boldsymbol{\Delta L}_{\mathbf{2}}(\mathbf{c m})$ & $\Delta \mathrm{L}_{\text {avg. }}(\mathbf{c m})$ \\
\hline 0 & 0.0 & 0.0 & 0.0 \\
\hline 30 & 1.0 & 1.3 & 1.2 \\
\hline 60 & 2.4 & 1.5 & 2.0 \\
\hline 90 & 2.8 & 1.7 & 2.3 \\
\hline. &. &. &. \\
\hline. &. &. &. \\
\hline. &. &. &. \\
\hline 870 & 1.4 & 0.8 & 1.1 \\
\hline 900 & 1.3 & 0.8 & 1.1 \\
\hline 930 & 1.4 & 0.8 & 1.1 \\
\hline 960 & 1.4 & 0.8 & 1.1 \\
\hline
\end{tabular}

Here, $\Delta L_{1}$ represents the saturation lag length in the first principal direction (the warp or direction of the major-axis of the flow ellipse), $\Delta L_{2}$ in the second direction, and the average of the two $\Delta L_{\text {avg. }}$. Plotting this data for the time series, as is shown in Figure 35 and Figure 36, reveals the same entrance flow effects present in Figure 29, and an increasingly linear relationship as time progresses. In fact, it appears that the farther away the flow becomes from the resin inlet, the tow saturation lag converges to a constant value. 


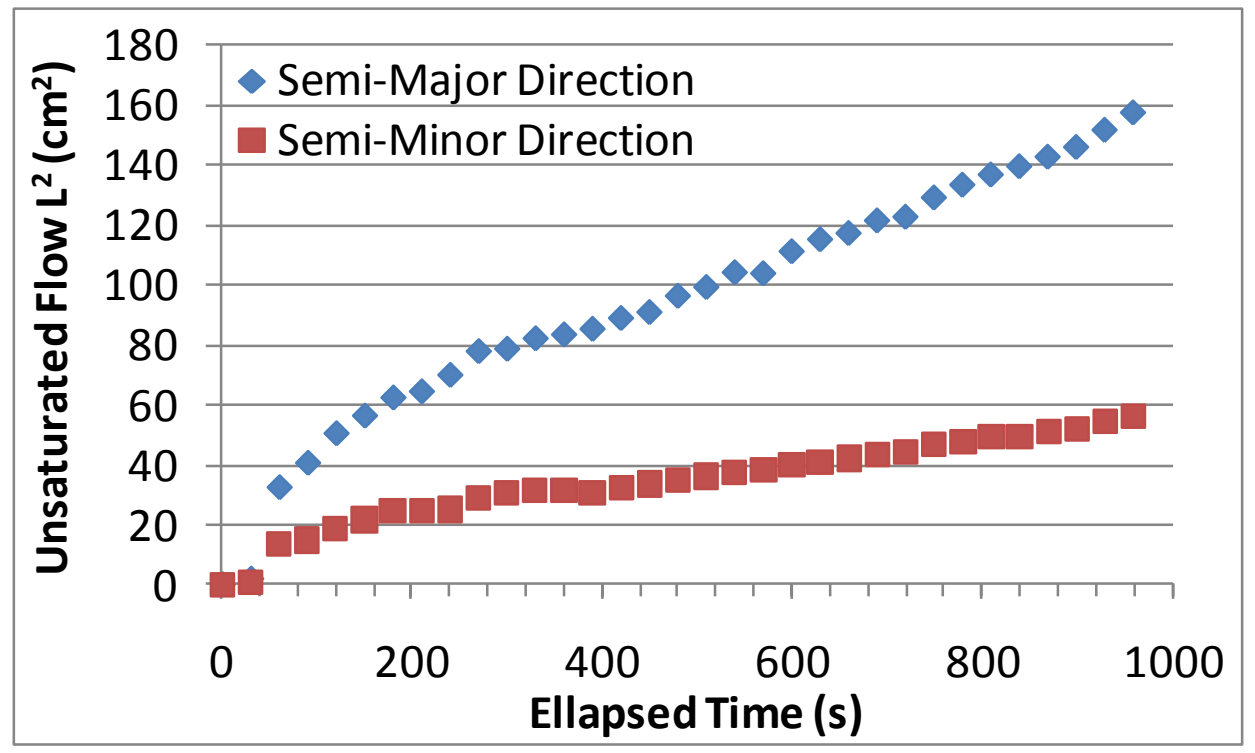

Figure 35: The linear nature of the unsaturated flow front lengths squared

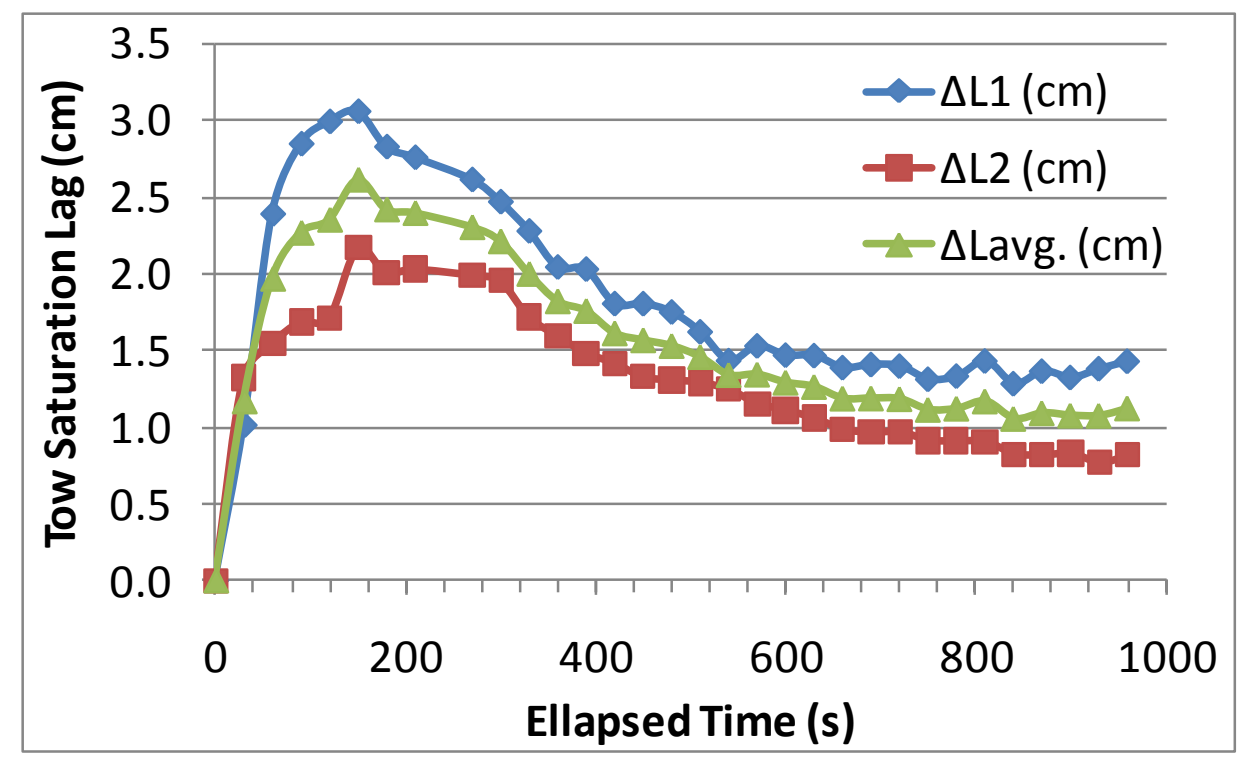

Figure 36: Tow saturation lag vs. time for a single experiment of $30^{\circ}$ induced fabric shear 
The tow saturation lag in the warp or flow front major-axis direction was always slightly larger than that in the second direction. For consistent results, the average lag of the two was taken and used for further calculations, but it is important to note that the tows are filling slower in the warp direction. This must lead us to believe that the warp tows are under a greater tensile load due to crimp as they are subject to more undulations being woven in between the higher pick count. Also, when we look at the anisotropy ratio (Figure 37), the ratio between the two principal flow lengths $R_{1}$ and $R_{2}$, we can see that the ratio $R_{1} / R_{2}$ increases with fabric shear. If this is true, we must assume that the orthogonal principal permeabilities $K_{1}$ and $K_{2}$ must follow a similar anisotropic relationship, increasing with fabric shear.

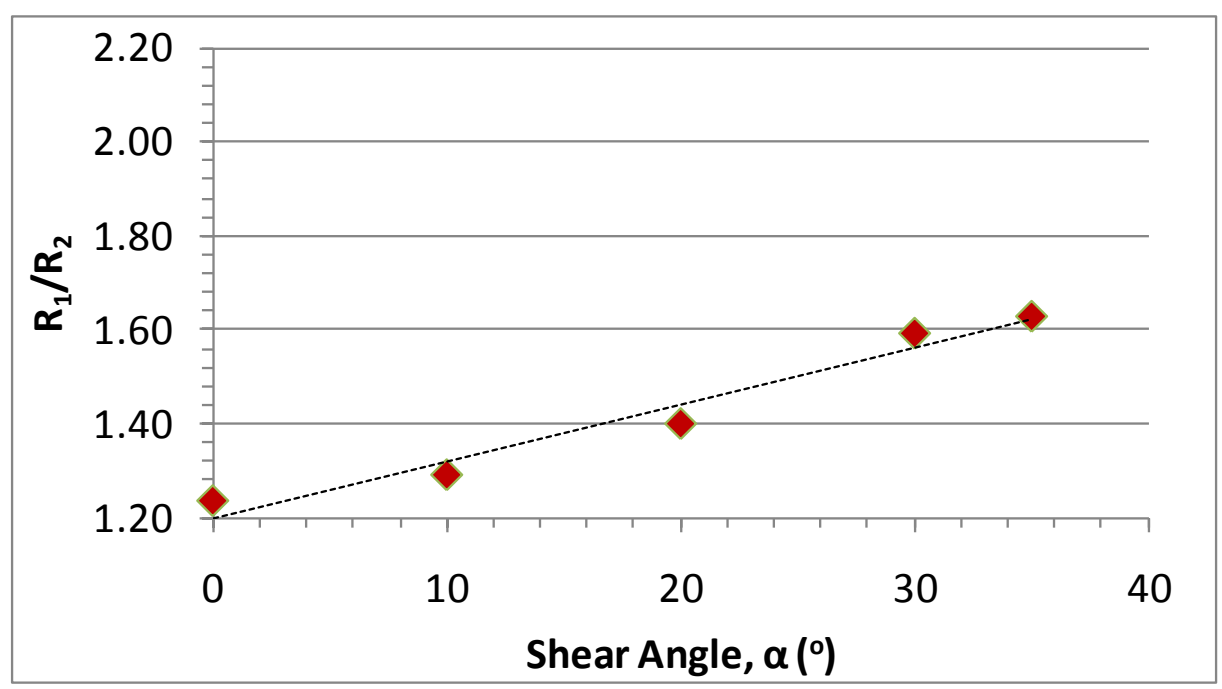

Figure 37: Anisotropy ratio of principal permeabilities as a function of fabric shear 
The slower bulk flow in the $K_{2}$, or semi-minor axis direction of the flow ellipse allows the fiber tows to saturate at a more similar rate than that in the warp direction. This may also explain why this phenomenon is witnessed. The assumption that the saturated flow lag is constant and not dependent on flow direction is used for simplicity with the numerical model. This assumption can later be relaxed, and with the use of the draping simulation information, specifically the warp, weft, and principal directions, one could model the directionally dependent tow permeability.

To quantify these results for each experiment, the harmonic mean of the average tow saturation lag for times where the flow front position is much greater than the diameter of the resin inlet is calculated. The harmonic mean is a suitable method to measure the central tendency of this data as it tends strongly toward the smallest values in a data set, mitigating the effect of large outliers and amplifying the effect of small ones. This is appropriate for this application upon visual inspection of Figure 36 when searching for the average tow saturation lag as time becomes very large, and we can be confident that all entrance effects are negligible. The harmonic mean puts more weight on the smaller values seen as time progresses through the experiment, and begins to neglect the larger values witnessed closer to the start of the experiment. This ensures that the values obtained are for after the lag reaches an asymptotic level and becomes constant. 
Table 5: Harmonic means and averages of tow saturation lag as a function of shear angle once the saturated flow lag becomes constant

\begin{tabular}{|c|c|c|c|c|c|}
\hline \multicolumn{7}{|c|}{ Tow Saturation Lag Behind Macro Flow Front } \\
\hline & \multicolumn{5}{|c|}{ Fabric Shear Angle } \\
\hline Trial & $\mathbf{0}^{\circ}$ & $\mathbf{1 0}^{\circ}$ & $\mathbf{2 0}^{\circ}$ & $\mathbf{3 0 ^ { \circ }}$ & $\mathbf{3 5}^{\circ}$ \\
\hline $\mathbf{1}$ & 0.60 & 0.77 & 0.86 & 1.36 & 3.96 \\
\hline $\mathbf{2}$ & 0.51 & 0.87 & 0.88 & 1.31 & 3.55 \\
\hline $\mathbf{3}$ & 0.52 & 0.77 & 1.07 & 1.90 & 3.63 \\
\hline $\mathbf{4}$ & 0.48 & & 0.98 & 1.41 & 3.23 \\
\hline Average Lag (cm) & $\mathbf{0 . 5 3}$ & $\mathbf{0 . 8 0}$ & $\mathbf{0 . 9 5}$ & $\mathbf{1 . 5 0}$ & $\mathbf{3 . 5 9}$ \\
\hline Standard Deviation $(\mathbf{c m})$ & $\mathbf{0 . 0 5}$ & $\mathbf{0 . 0 6}$ & $\mathbf{0 . 1 0}$ & $\mathbf{0 . 2 7}$ & $\mathbf{0 . 3 0}$ \\
\hline
\end{tabular}

This method of data capture and analysis was conducted for all experiments, resulting in the outcomes seen in Table 5. The average tow saturation lag of the experimental trial harmonic means was taken for each degree of the deformed fabric. It can also be seen that the standard deviations are relatively low (typically less than $10 \%$ of the mean) for the different shear angles, but with more variability as fabric shear increases. The data shows an exponential trend in which the tow saturation lag increases as a function of fabric shear angle (Figure 38), as was assumed by the initial hypothesis. As can be seen from the data, it becomes increasingly important to account for the change in tow permeability with increased fabric shear as the tow saturation lag is significant. This raw data can now be used to fully characterize the specific fabric using techniques later discussed in the modeling section of Section 3.5. 


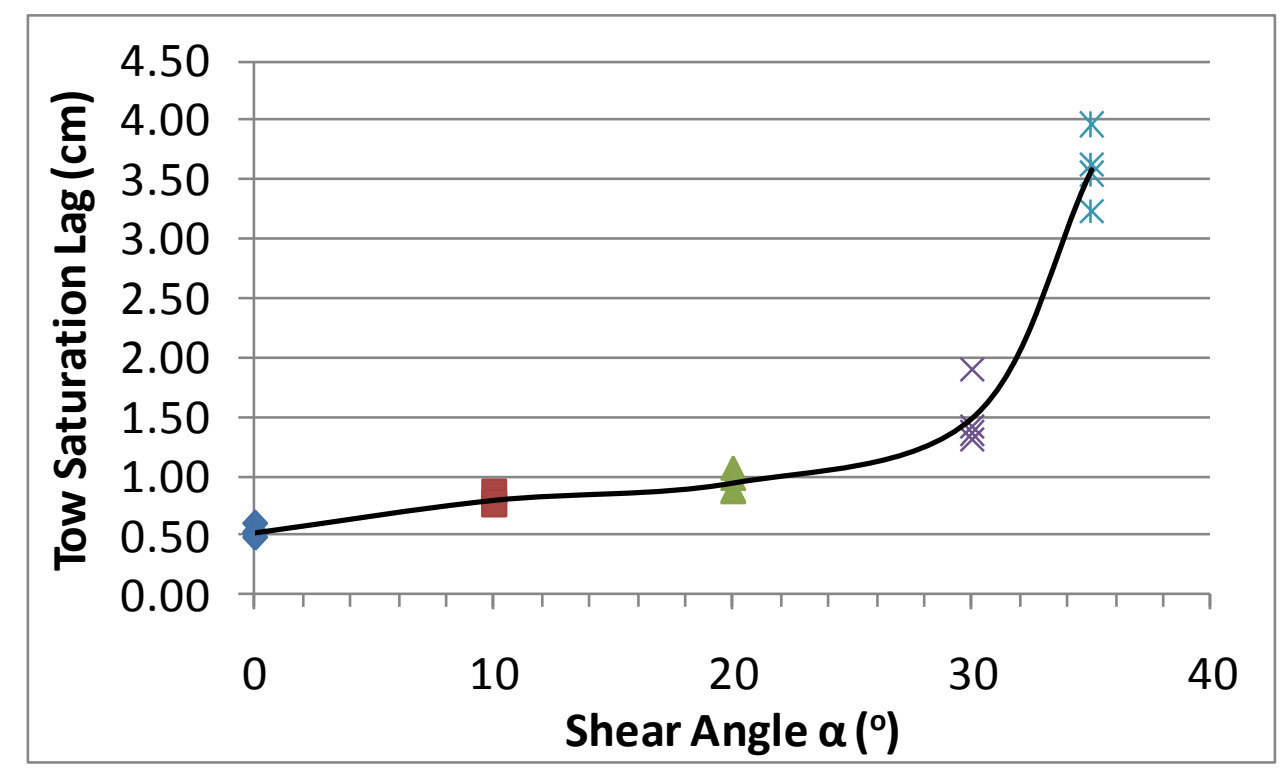

Figure 38: Experimental data for tow saturation lag vs. induced shear angle.

This raw data can be non-dimensionalized in a way in which results obtained from any experiment, with any given fabric and resin system, can be compared and analyzed. To do this, several assumptions must be made. The tow saturation lag length $\Delta L$ can be non-dimensionalized with respect to the process parameters according to:

$$
\Delta L^{*}=\frac{\Delta L}{h} \frac{P t}{\mu}
$$

where $P$ is the applied process pressure, $t$ is the elapsed time, and $\mu$ is the resin viscosity. The length $h$ is half the tow thickness, as is described in Figure 6 as the distance the liquid resin must travel to completely saturate the fiber tow bundle. These values are then transformed into a percentage different from the undeformed, or 
unsheared fabric case. This non-dimensionalized parameter can then be plotted against a non-dimensionalized time value:

$$
t^{*}=\frac{t}{t_{\text {fill }}}
$$

where $t_{\text {fill }}$ is the time it takes for the resin flow to "fill" the desired preform. For the purpose of analyzing these experiments, $t_{\text {fil }}$ has been designated to be the time for the major-axis length of the saturated flow ellipse to have reached an arbitrary length of 8 inches. Figure 39 shows the difference of the non-dimensionalized tow saturation length $L^{*}$ plotted against the non-dimensionalized time parameter $t^{*}$ for single experimental trials spanning the range of fabric shear angles investigated. It can be seen that with increased fabric shear, the lag increases compared to the baseline unsheared case. Again, this normalized data confirms the finding that the gap between the macro flow front and saturated fiber region increases as a function of shear angle.

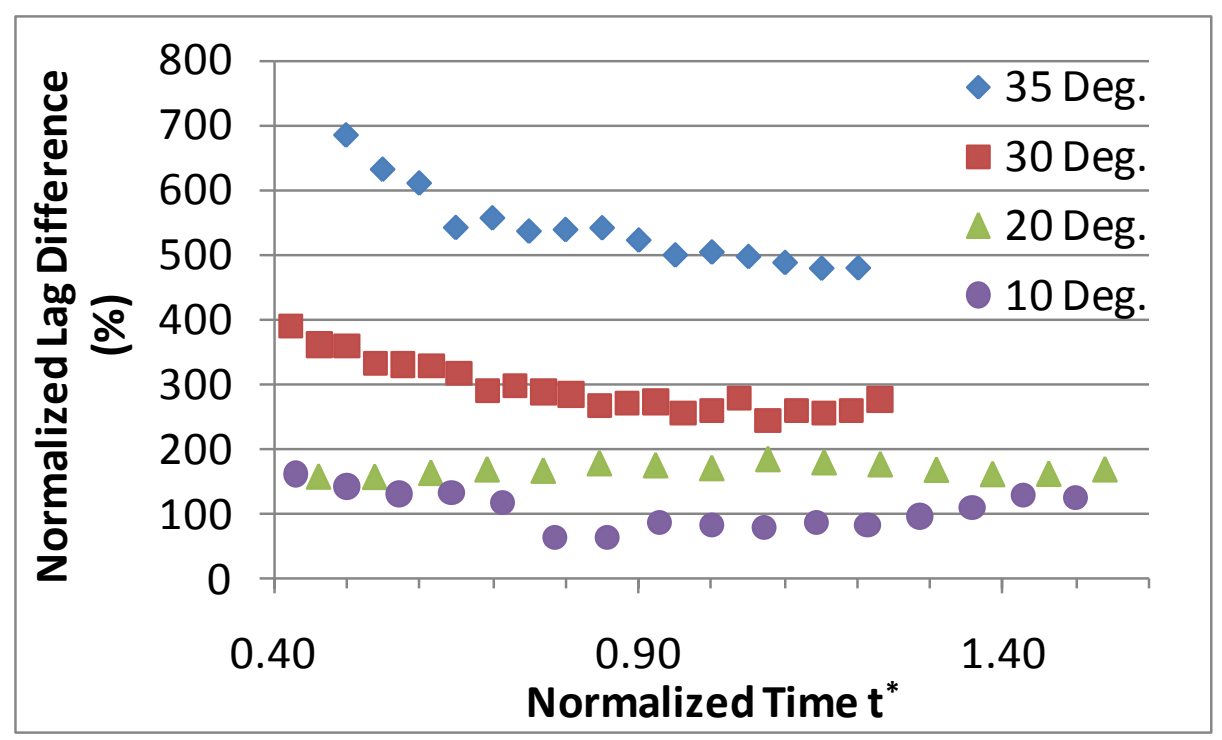

Figure 39: Non-dimensionalized plot of tow saturation lag data 
To predict the slow filling of the fiber tows in a flow simulation model, the following is needed

(1) Simulation that can separately account for fiber tow permeability and fabric permeability. Liquid Injection Molding Simulation does this but can only model the fiber tow filling in an average sense with an isotropic permeability.

(2) One needs to provide the input for fabric permeability and tow permeability as a function of change in fiber volume fraction

(3) One needs to provide the relationship between shear angle and the fiber volume fraction.

In the next chapter, we will develop the numerical model and use the experimental results to propose the relationship between change in permeability of the fiber tow to the change in fiber volume fraction by matching the experimental results with the predicted ones.

We now have all of the necessary tools and information to fully characterize the tow permeability of the fabric for use in our numerical model. After the model and mesh have been created, they will be used along with several governing equations to determine how fabric and tow permeability change with fabric shear.

\subsection{Bulk Permeability Characterization}

The previous chapters have described in detail how the numerical model is constructed, and how to obtain the necessary raw experimental data necessary for 
material characterization. It is this fully characterized material input that will be critical to the numerical model's accuracy. In order to precisely predict dual scale flow in a deformed preforms, one must characterize porosity and permeability change for both the bulk fabric and fiber tow bundles as a function of local fabric shear angle. Using the raw experimental data recorded in the previous chapter, we will now look into fully characterizing the specific fabric of interest. This process must be repeated for each new fabric to be used with the proposed numerical model.

The first step in characterization is to develop a model to describe how the bulk fabric permeability changes in both principal directions with induced fabric shear. This is obtained from the measured undeformed bulk fabric permeability $K_{x x}$, as well as the undeformed $\left(0^{\circ}\right.$ shear) flow front progression data found from the radial flow experiments and the image processing code. First, however, we need to determine the warp permeability $K_{x x}$ for the VARTM experiment, or more specifically for the FVF induced by the compressive vacuum pressure. We cannot assume that the VARTM fiber volume fraction, and therefore permeability is the same as any of those previous measured using the RTM experimental procedure.

It had been measured for 4 experiments that the undeformed fiber volume fraction was $53 \%$ for the VARTM process. Although this is close to the mid-range FVF measured with the RTM experiment (54\%), we can be more precise in estimating the directional fabric permeability using a simple relationship which has already been discussed. Assuming all other experimental parameters are negligible, we will use the permeability relationship given by the Kozeny-Carmen equation and the RTM permeability results found previously to determine the undeformed warp permeability $K_{x x}$ for $53 \% \mathrm{FVF}$. 


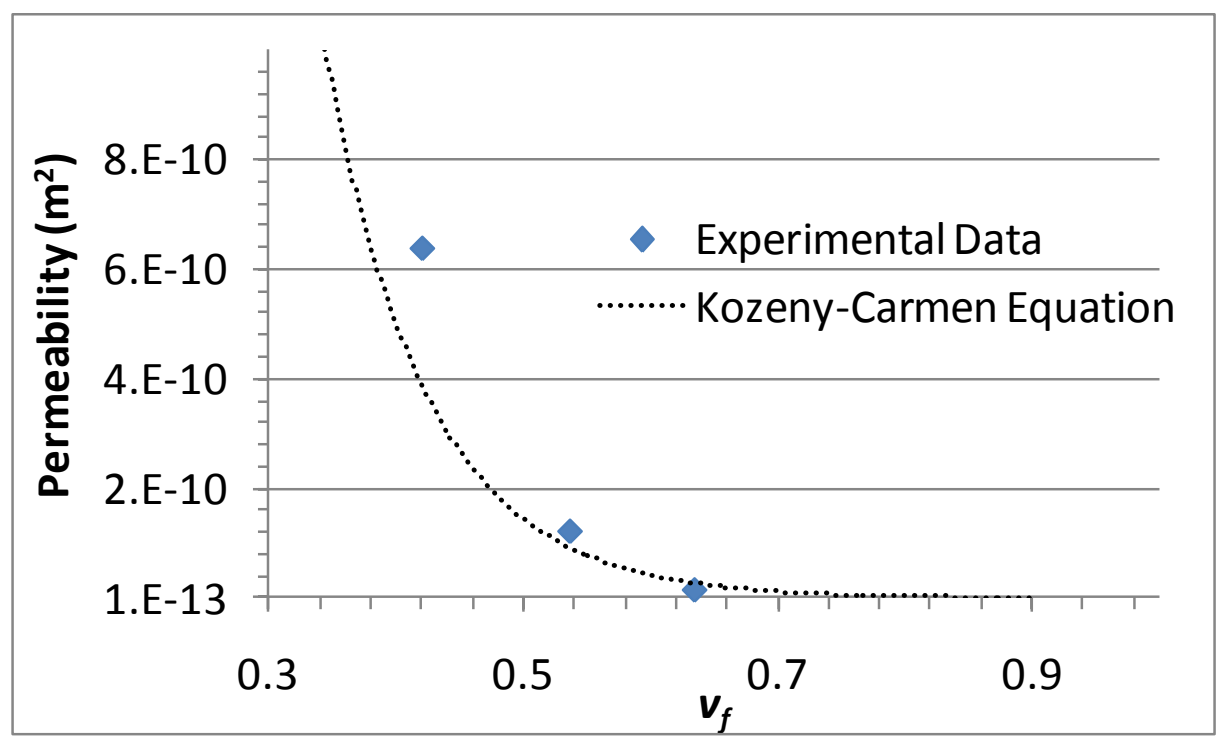

Figure 40: Kozeny-Carmen equation fit to the experimental data

The Kozeny-Carmen predicts directional permeability as a function of an experimentally measured constant, $C$, and fiber volume fraction.

$$
K=C \frac{\left(1-v_{f}\right)^{3}}{v_{f}^{2}}
$$

The constant $C$ has already been determined from the performed RTM experiments, and we are seeking the permeability $K_{x x}$ for a known $v_{f}=53 \%$. It can be seen below in Figure 40 that the simple Kozeny-Carmen relationship fits the experimental data well, especially for fiber volume fractions close to the one of interest. This equation estimates that the VARTM $K_{x x}\left(K_{l}\right)$ bulk permeability to be approximately $1.2 \times 10^{-10}$ $\mathrm{m}^{2}$. 
Now with the permeability in the warp direction know for the case of unsheared fabric, we can determine $K_{y y}$ or $K_{2}$. For each experiment, the semi-major and minor axis lengths were recorded for each time step. We will now use this information to calculate $K_{y y}$. It has been discussed earlier in Equation (3) that the ratio of principal permeabilities is proportionate to the square of the ratio of the flow ellipse axis lengths. More specifically:

$$
\frac{K_{1}}{K_{2}}=\frac{R_{1}^{2}}{R_{2}^{2}}
$$

where $R_{1}$ and $R_{2}$ (previously a and $\mathrm{b}$ ) are the experimentally measured semi-major and semi-minor elliptical flow front lengths, respectively. With $K_{l}$ known, we can easily calculate $K_{2}$ with the measured values for each experiment. $K_{2}$ was determined to have an average value of $8.6 \times 10^{-11} \mathrm{~m}^{2}$ with a standard deviation of $1.18 \times 10^{-11} \mathrm{~m}^{2}$ from the four experiments in which it was measured.

This same procedure was followed for the experimental trials with induced fabric shear. The preform fiber volume fraction was calculated using Equation 1 and the induced shear angle. Next, the permeability $K_{l}$ was estimated using the calculated FVF and the Kozeny-Carmen equation. Again, $K_{2}$ was calculated using the estimated $K_{1}$ and the experimentally observed anisotropy ratio $R_{l} / R_{2}$ for each given experimental set. By this method, the bulk fabric permeabilities were calculated and are plotted in Figure 41. 


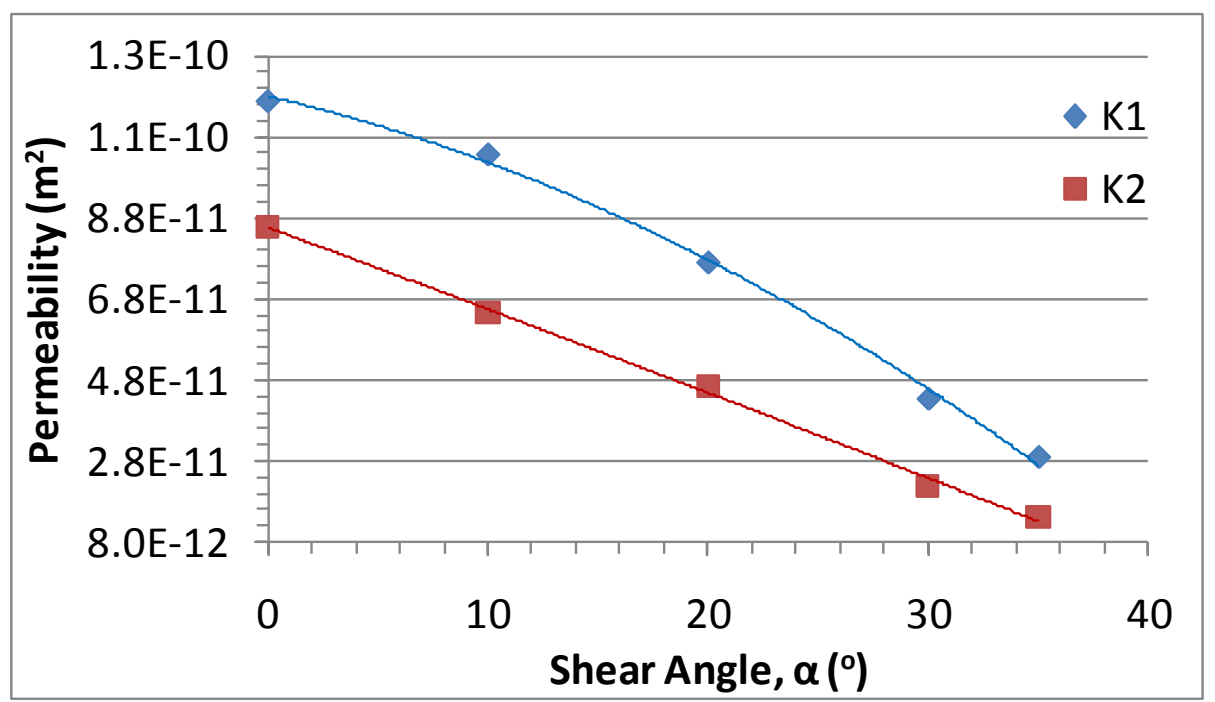

Figure 41: Calculated bulk principal permeabilities for a range of fabric shear angles for 24.5 oz ShieldStrand-S fabric

It is desired to express permeabilities $K_{1}$ and $K_{2}$ as a function of fabric shear angle which can then be used in the simulation. We propose a constitutive equation of the form:

$$
K_{1,2}=\theta_{0}(1-\alpha)^{\theta_{1}}
$$

with the shear angle $\alpha$ in radians, and two empirical parameters $\theta_{0}$ and $\theta_{1}$. For both principal permeabilities, $\theta_{0}$ is equivalent to the undeformed fabric permeability $K_{1,2}^{0^{o}}$, or $K_{x x}$ and $K_{y y}$ as listed in Table 6. 
Table 6: $\quad$ Empirical parameters for $K_{1}$ and $K_{2}$

\begin{tabular}{|c|c|c|}
\hline \multicolumn{1}{|c|}{$\boldsymbol{\theta}_{\mathbf{0}}$} & $\boldsymbol{\theta}_{\mathbf{1}}$ \\
\cline { 2 - 3 } $\boldsymbol{K}_{\mathbf{1}}$ & $1.2 \mathrm{E}-10$ & 1.34 \\
\hline $\mathbf{K}_{\mathbf{2}}$ & $8.6 \mathrm{E}-11$ & 1.87 \\
\hline
\end{tabular}

The proposed empirical models correlate to the calculated permeability values acceptably, as can be seen from Figure 42 below.

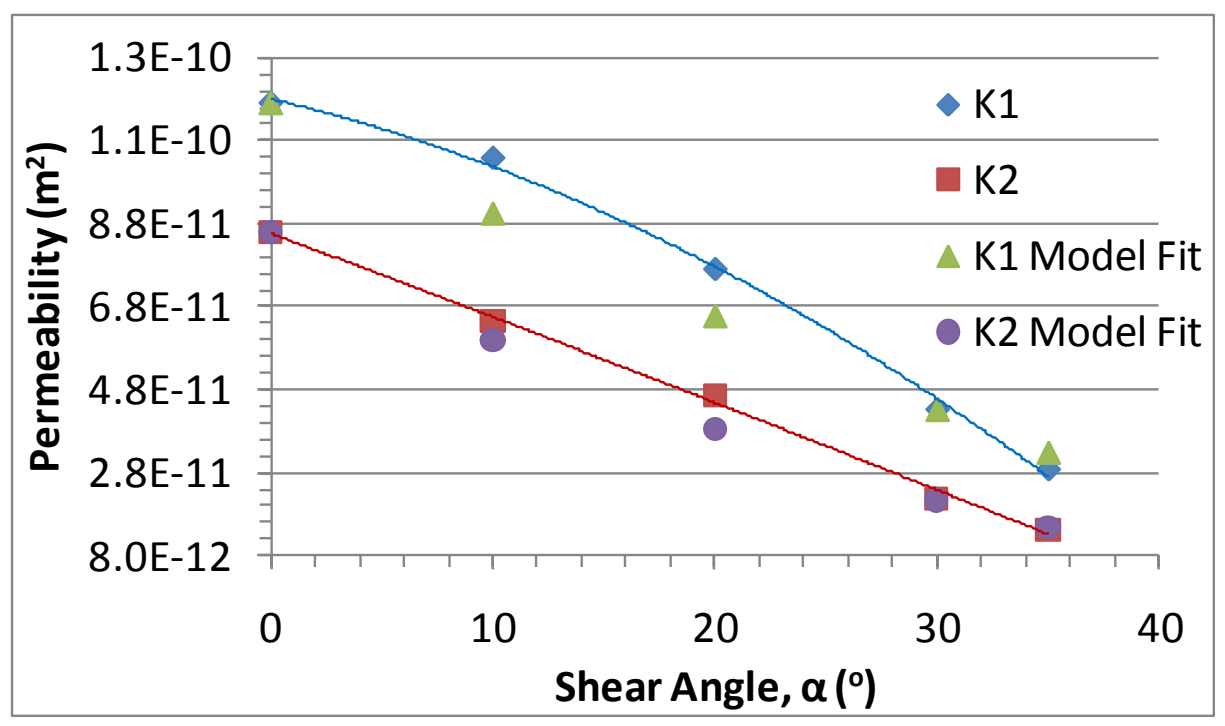

Figure 42: Correlation between calculated permeability values and the proposed empirical model data

As discussed previously, the anisotropy ratio between the principal permeabilities is of interest. We are now able to determine how this anisotropic 
relationship changes with fabric shear. As anticipated, the ratio $K_{1} / K_{2}$ increases along with fabric shear, as is seen below.

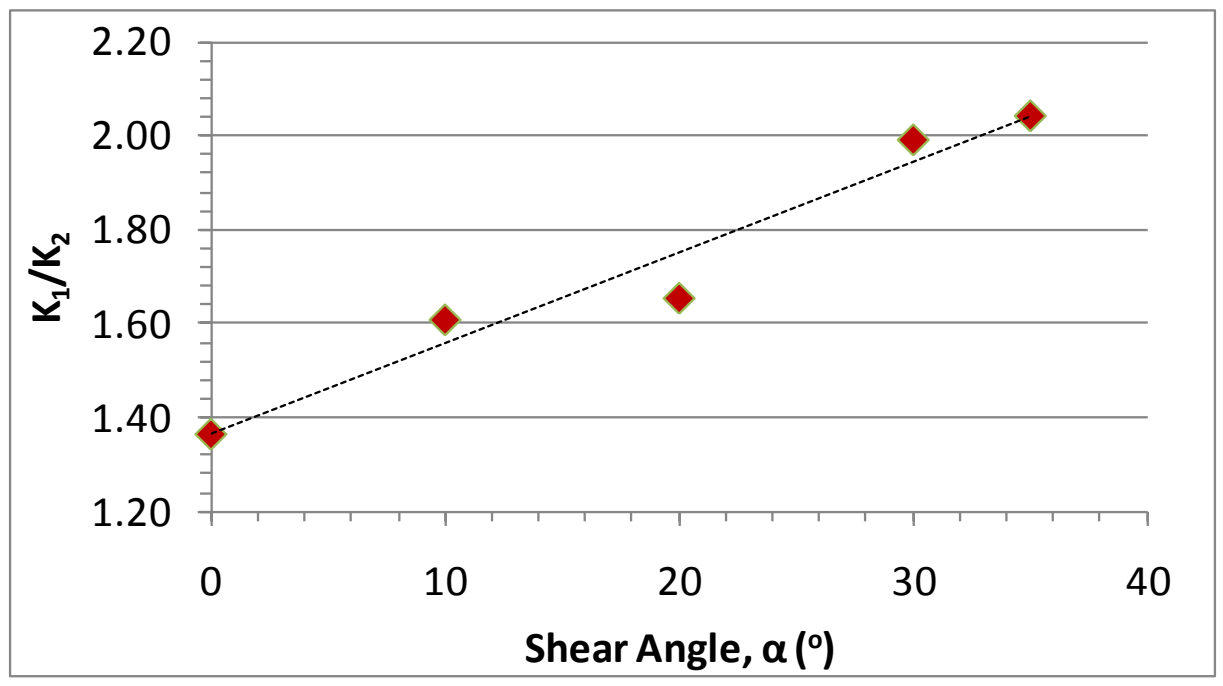

Figure 43: Anisotropic relationship between the principal permeabilities increasing as a function of fabric shear

We now have the bulk fabric permeabilities and porosities characterized for a range of fabric shear angles using experimental data fitted to empirical mathematical relationships.

\subsection{Tow Permeability Characterization}

A procedure was devised to investigate how the fabric shear affects the other half of dual scale flow: tow permeability and saturation. In order to accomplish this, we input the bulk permeability as a function of the shear angle into the LIMS simulation. We can track the saturated and unsaturated flow front as a function of tow 
permeability in the simulation once we input the fiber tow permeability for the one dimensional elements in the mesh. Thus this allows us to relate the lag between the saturated and unsaturated flow front to the tow permeability value we input in the simulation. The tow permeability used for the one dimensional elements that gives us the best fit between the lag observed in the experiments and the simulation for that specific shear angle is declared as the permeability of the fiber tow for that degree of shear.

A very fine $2 \mathrm{D}$ mesh was created with $0.1 "(2.54 \mathrm{~mm})$ square elements in order to track the difference between the saturated and unsaturated flow fronts. The simulations were run to exactly replicate the corresponding experiments, with the same number of fabric plies, and using the same resin viscosity for each trial. Five nodes in the center of the mesh were designated as resin gates, replicating the $5 \mathrm{~mm}$ radius injection line used in the experiments.
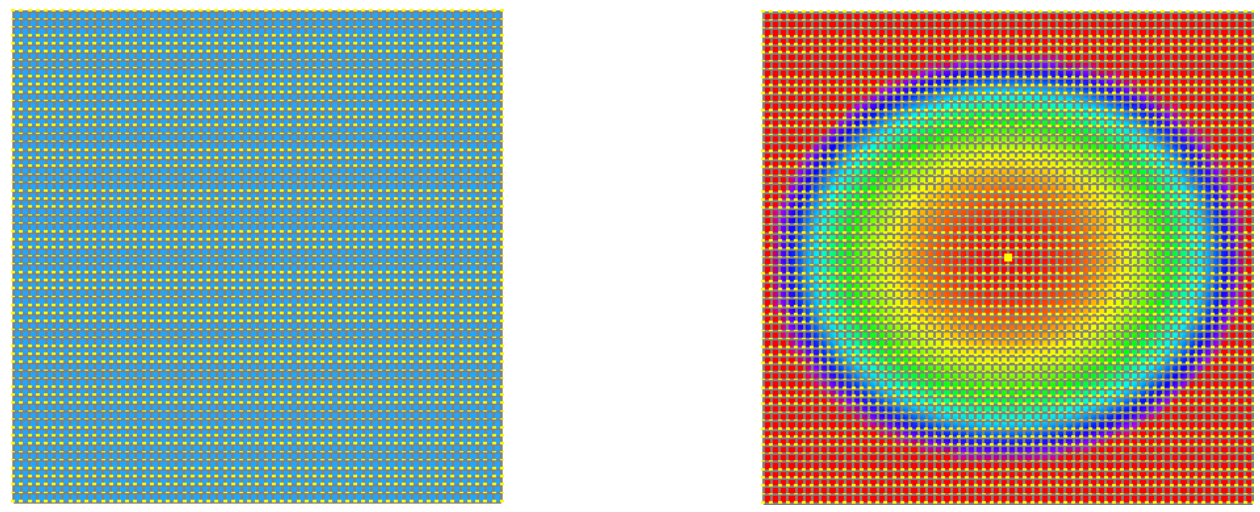

Figure 44: ( $a$ - left) Fine mesh used to track tow impregnation and ( $b-$ right) mold filling times predicted by the finite element simulation 
For each experimental trial, the model tow permeability was adjusted until the appropriate saturation lag as measured in Section 3.3 matched the model results. Using this approach, we were able to determine how fabric shear influences fiber tow permeability and characterize the fiber tow permeability as a function of fabric shear.

Table 7: $\quad$ Tow permeability as a function of fabric shear

\begin{tabular}{|c|c|}
\hline Shear Angle $\boldsymbol{\alpha}\left(^{\circ}\right)$ & $\mathbf{K}_{\text {tow }}\left(\mathbf{m}^{2}\right)$ \\
\hline 0 & $1.00 \mathrm{E}-12$ \\
\hline 10 & $2.00 \mathrm{E}-13$ \\
\hline 20 & $1.00 \mathrm{E}-13$ \\
\hline 30 & $2.50 \mathrm{E}-14$ \\
\hline 35 & $4.00 \mathrm{E}-15$ \\
\hline
\end{tabular}






Figure 45: Tow permeability as a function of fabric shear angle

It was of interest to see how sensitive the numerical model is to the adjustment of the 1D element fiber bundle permeability. To conduct this study, 20\% and $50 \%$ of the calculated tow permeabilities were investigated to see how the reduction in tow permeability, with all other model parameters held constant, affects the tow saturation. Table 8 and Figure 46 show how the lag between the saturated and unsaturated flow fronts are affected by the reduction in tow permeability for the range of shear angles. 
Table 8: Change in tow saturation lag versus shear angle for set reductions in tow permeability

\begin{tabular}{|c|c|c|c|c|c|c|c|c|}
\hline \multirow{2}{*}{$\begin{array}{c}\text { Shear Angle } \\
\alpha\left({ }^{\circ}\right)\end{array}$} & \multicolumn{2}{|c|}{$K_{\text {tow }}$} & \multicolumn{3}{|c|}{$50 \% \mathrm{~K}_{\text {tow }}$} & \multicolumn{3}{|c|}{$20 \% \mathrm{~K}_{\text {tow }}$} \\
\hline & $K_{\text {tow }}\left(\mathrm{m}^{2}\right)$ & $\Delta \mathrm{L}(\mathrm{cm})$ & $K_{\text {tow, } 50 \%}\left(\mathrm{~m}^{2}\right)$ & $\Delta \mathrm{L}(\mathrm{cm})$ & $\%$ Difference & $\mathrm{K}_{\text {tow, } 500 \%}\left(\mathrm{~m}^{2}\right)$ & $\Delta \mathrm{L}(\mathrm{cm})$ & \% Difference \\
\hline 0 & $1.0 \mathrm{E}-12$ & 0.53 & $5.0 \mathrm{E}-13$ & 1.02 & $62.9 \%$ & $2.0 \mathrm{E}-13$ & 1.27 & $82.2 \%$ \\
\hline 10 & $2.0 \mathrm{E}-13$ & 0.80 & $1.0 \mathrm{E}-13$ & 1.27 & $45.4 \%$ & $4.0 \mathrm{E}-14$ & 1.52 & $62.3 \%$ \\
\hline 20 & $1.0 \mathrm{E}-13$ & 0.95 & $5.0 \mathrm{E}-14$ & 1.78 & $60.7 \%$ & 2.0E-14 & 2.16 & $77.8 \%$ \\
\hline 30 & $2.5 \mathrm{E}-14$ & 1.50 & 1.3E-14 & 2.54 & $51.5 \%$ & $5.0 \mathrm{E}-15$ & 3.30 & $75.1 \%$ \\
\hline 35 & 4.0E-15 & 3.59 & $2.0 \mathrm{E}-15$ & 4.57 & $24.1 \%$ & 8.0E-16 & 6.10 & $51.7 \%$ \\
\hline
\end{tabular}

It can be seen that $50 \%$ reduction in tow permeability is met by a significant increase in the length $\Delta L$ seen between the bulk and saturated flow fronts in the numerical results. It can be concluded that it is very important to properly characterize the tow permeability in order to properly model tow saturation as a function of fabric shear. 


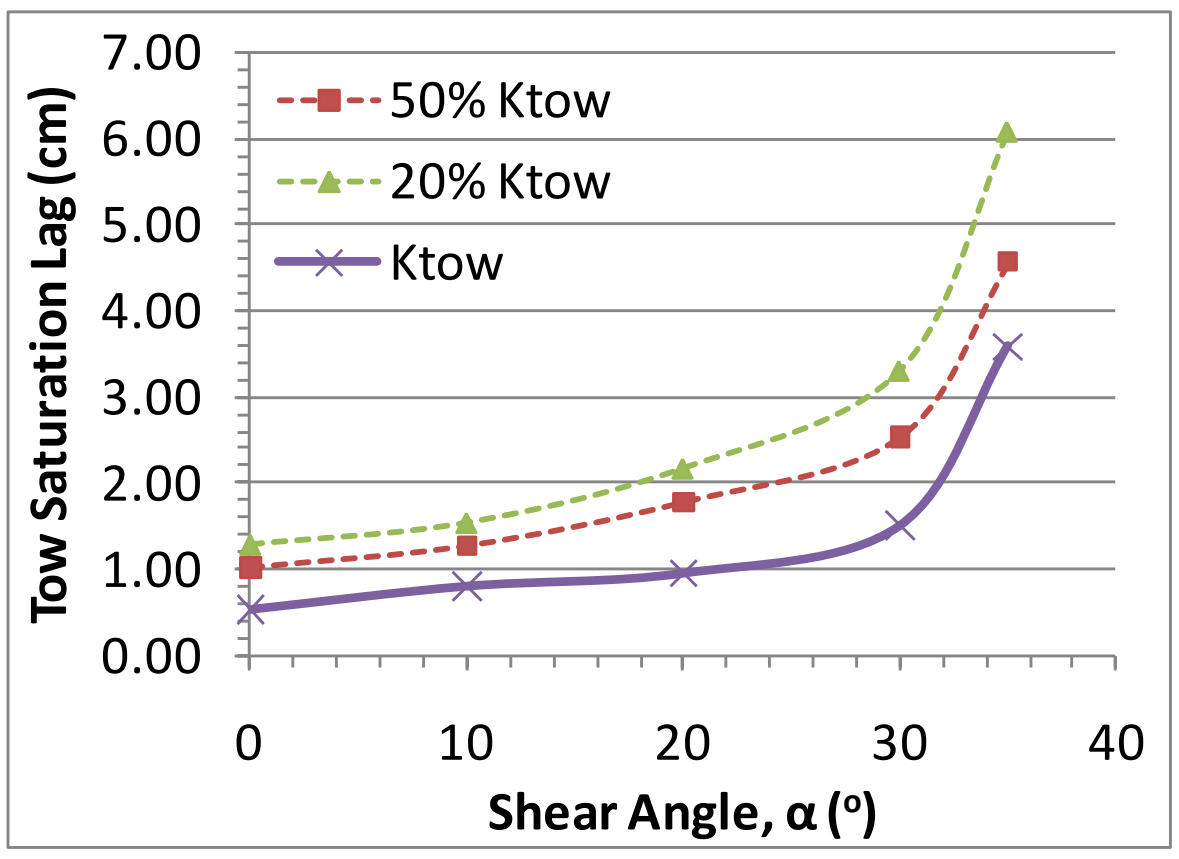

Figure 46: Change in tow saturation lag as tow permeability is varied

Once again, it is desirable to seek a constitutive model in the form of that of Equation 24, capable of expressing these permeability values as a function of fabric shear angle. Once again, $\theta_{0}$ is equivalent to the undeformed $K_{\text {tow }}^{0^{o}}=1 \times 10^{-12} \mathrm{~m}^{2}$, and $\theta_{1}=5.8$. These parameters result in the excellent fit seen in Figure 47. The values for tow permeability vary from $1 \times 10^{-12} \mathrm{~m}^{2}$ for the undeformed fabric, to $4 \times 10^{-15} \mathrm{~m}^{2}$ for fabric with $35^{\circ}$ induced shear. 


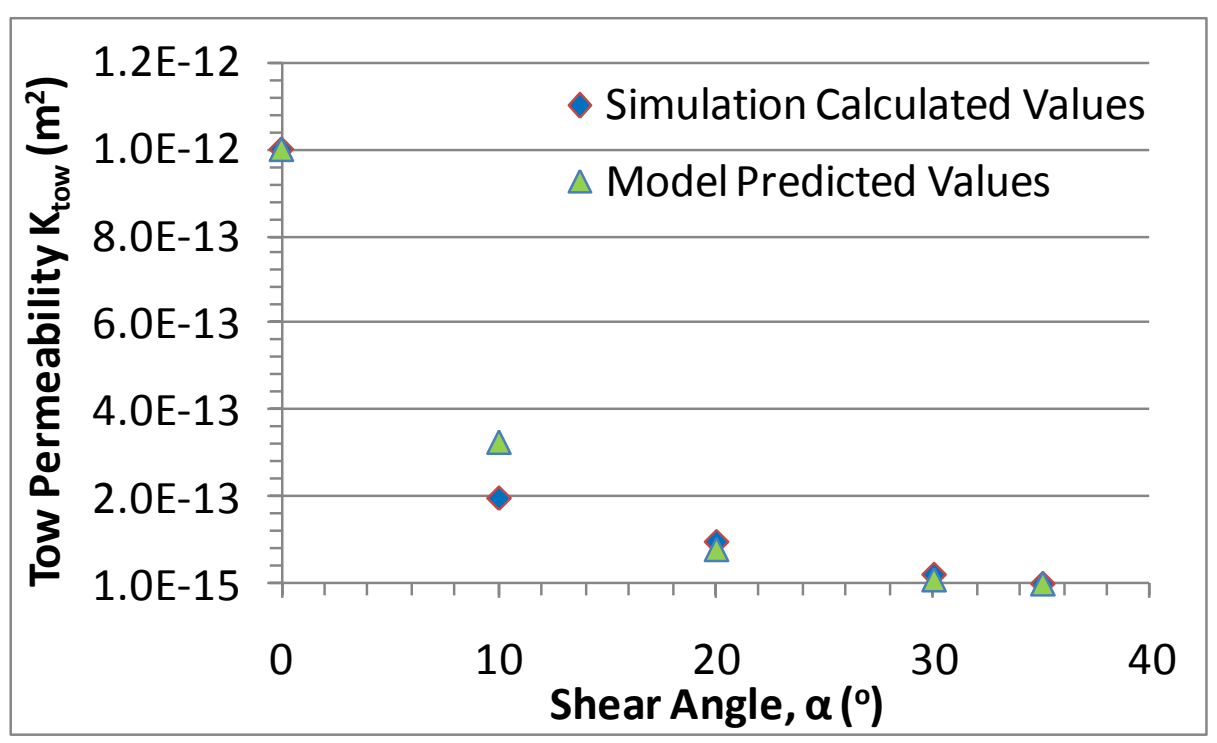

Figure 47: Correlation between calculated tow permeability data and the proposed fit model values 


\section{Chapter 4}

\section{INFLUENCE OF FIBER TOW SHEAR ON RESIN FLOW}

\subsection{Model Validation}

To study the effects that fabric shear has on dual scale flow and mold filling, a radial injection simulation on a flat, square mesh with uniformly induced shear was conducted to replicate and confirm the experimental findings. Fabric shear was induced in the flat plate by entering a constant shear angle in all equations during mesh generation. This set shearing angle was used to calculate bulk and fiber tow volume fraction, permeability, and principal flow directions. The simulation results were both visually and quantitatively compared with the experiments. Because the simulation input information is based upon the experimental data (Table 9), the results should coincide.

Table 9: Empirical permeability parameters used for principal fabric $\left(K_{1}, K_{2}\right)$ and fiber tow $\left(K_{\text {tow }}\right)$ in the numerical model simulations

\begin{tabular}{|c|c|c|}
\hline & $\theta_{0}$ & $\theta_{1}$ \\
\hline$K_{1}$ & $1.2 \mathrm{E}-10$ & 1.34 \\
\hline$K_{2}$ & 8.6E-11 & 1.87 \\
\hline $\mathbf{K}_{\text {tow }}$ & 1.0E-12 & 5.80 \\
\hline
\end{tabular}


For each discrete shear angle of interest, an experimental trial was chosen to verify that the numerical model is capable of accurately predicting both the unsaturated and saturated flow front progression. For each trial, the appropriate fabric shear angle and experimental viscosity were entered into the model. Next, the simulation was run for a desired time step, for which the experimental flow front lengths $R_{1}$ and $R_{2}$ for both the saturated and bulk flow regions are all known. For all experiments compared in this manner, the processing time required for the semi-major axis of the bulk flow, $R_{1 \text {, unsaturated, }}$ to reach a length of approximately 3 inches was chosen. The resulting comparison of experimental flow fronts to those generated by the numerical model can be seen below in Figure 48 .
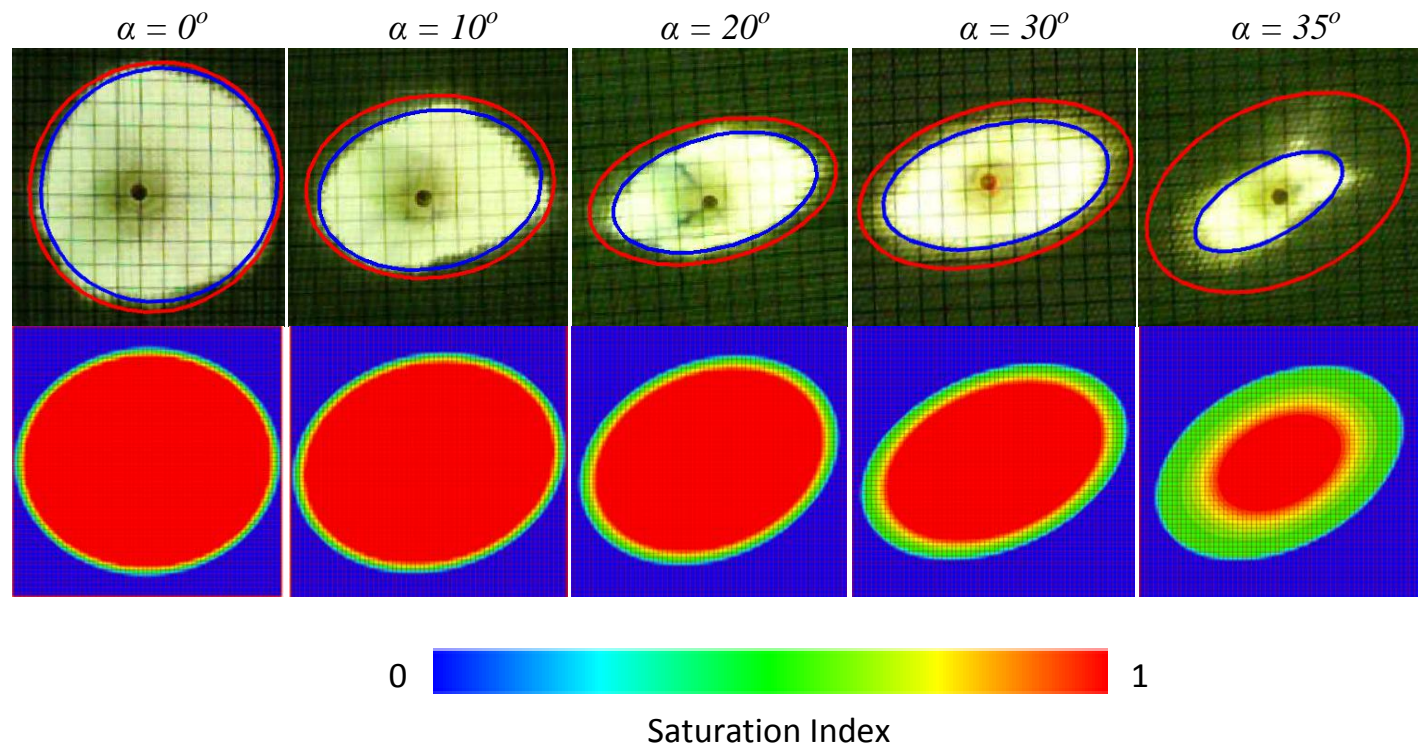

1

Figure 48: Comparison between experimental and simulation flow fronts for set time steps. All images represent flow in an approximately 3 " square. 
The simulations appear to match the experimental data well. To confirm this in a quantitative manner, the time required for the simulation flow fronts to reach a node a precise distance from the injection site was investigated. The nodes chosen again correspond to known flow distances that had been observed experimentally. Typically, the simulation fill times were less than $10 \%$ different from the measured data. The average percent difference between the measured experimental fill times and the matching simulation fill times for the four experiments for each case of induced fabric shear are listed in Table 10.

Table 10: Average difference between witnessed experimental and simulation fill times for the flow to reach a node three inches from the resin inlet

\begin{tabular}{|c|c|}
\hline Shear Angle, $\boldsymbol{\alpha} \mathbf{(}^{\mathbf{}}$ ) & Avgerage Difference (\%) \\
\hline 0 & $-8.5 \%$ \\
\hline 10 & $8.8 \%$ \\
\hline 20 & $0.3 \%$ \\
\hline 30 & $7.9 \%$ \\
\hline 35 & $4.1 \%$ \\
\hline
\end{tabular}

Thus, the numerical model developed is adequate for addressing the dual scale flow nature associated with dual scale fabrics in which the fiber tow porosity and permeability are affected by the shear. Using the characterized fabric information, we were able to accurately model the fiber tow saturation lag behind the bulk flow front as a function of fabric shear. We were also able to sufficiently verify mold filling 
times (in this case, the time for the fluid flow to reach a node three inches away from the resin inlet) within $10 \%$ of the observed values.

\subsection{Quantifying the Influence of Fiber Tow Shear on Mold Filling}

Now that this method has been successfully proven, we will continue by examining how this model has added benefit over the previously developed numerical techniques. In order to do this, we will compare the results from three types of numerical simulations to discuss the effects of accounting for shear deformation on fiber tows. The fabric properties used in the simulation will be used from equations (24) with the constants determined for the bulk fabric permeability and the tow permeability as listed in Table 9 and plotted in Figure 9, Figure 42, Figure 47. Results from the three cases will be compared to highlight the importance of accounting for the dual scale flow during shearing and also addressing the influence of fiber tow shearing on flow:

(i) Baseline: Two-dimensional mesh elements which are adjusted due to the effect of shear on bulk fiber volume fraction and permeability (no dual scale flow).

(ii) No Tow Shearing: Two-dimensional mesh elements, the properties (permeability, fiber volume fraction and orientation) are modified to account for the shear effects. Dual scale flow is simulated with the addition of one-dimensional tow elements in which the effect of shear is ignored. Hence the tow permeability is held constant at the same undeformed tow permeability value for all shear angles at $1 \times 10^{-12} \mathrm{~m}^{2}$ 
(iii) Tow Shearing: Two-dimensional and one-dimensional tow element properties are modified according to Equation (24) and Table 9 to account for the shear effects.

Case (i) is the baseline model. This type of simulation has been well established by previous work. Case (ii) accounts for the same physics as in simulation (i), but adds a constant sink term to account for the dual scale flow throughout the mold to account for tow filling. This sink term will have an effect on the resin flow front and will create an unsaturated front and also change the pressure profile throughout the injection time span.

Case (iii) is the original contribution of this thesis. Results of filling times and tow saturation from simulations (iii) will be compared with cases (i) and (ii) for the fabric behavior chosen as shown in Figure 9 to underline the importance of including the tow shearing in dual scale mold filling as a function of fabric shear angle. For case (iii) the fiber tow sink term will vary as the induced shear angle is changed as proposed by the empirical Equation (24). The same inputs for material property curves and draping simulation were used in all three cases. Models were created using induced fabric shear angles of $0^{\circ}, 10^{\circ}, 20^{\circ}, 30^{\circ}, 35^{\circ}$ to determine how mold filling changed as a function of shear angle. It was assumed that shear of more than $35^{\circ}$ will cause slippage of the tows and make the FiberSIM model results suspect. 

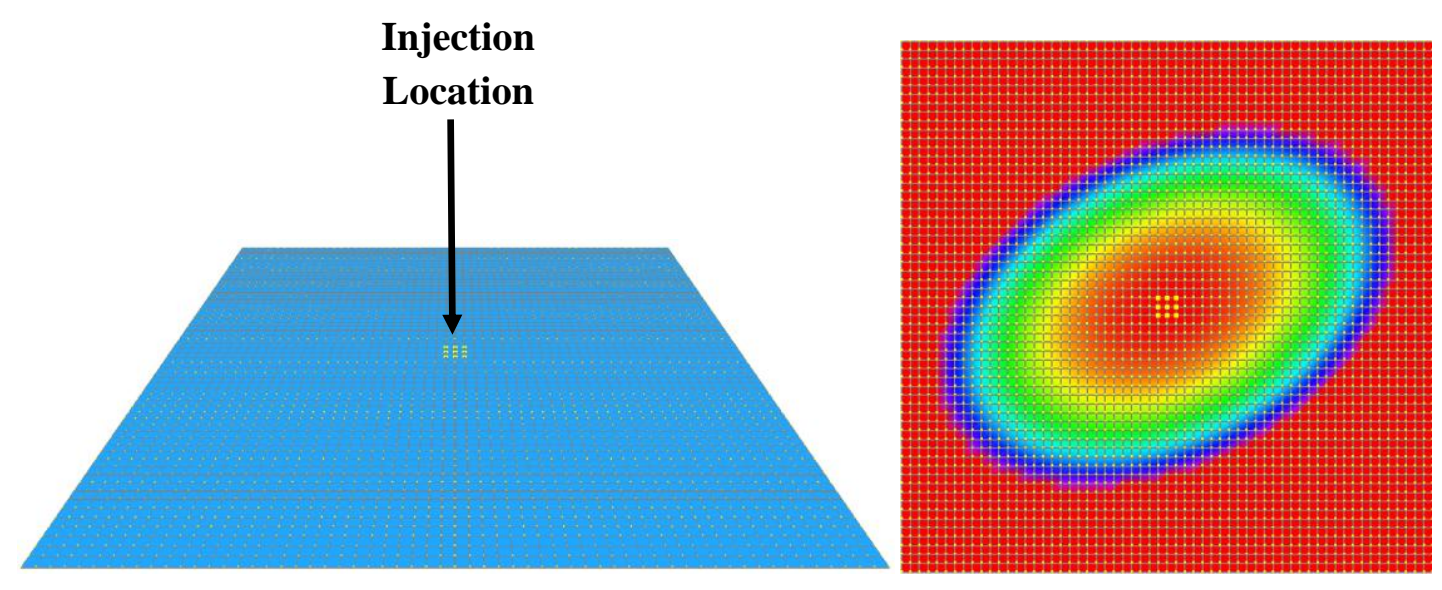

Figure 49: ( $a$ - left) Location of injection gate for radial flow simulation and $(b-$ right) radial flow front progression vs. time. Flow ellipse rotated due to induced shear angle

Figure 49 represent how each finite element simulation is performed. The same fine mesh, representing a 6" square preform, with 0.1 " quad elements is used throughout the following experiments. The viscous resin flow (viscosity of $0.5 \mathrm{~Pa}-\mathrm{s}$ ) is driven by a constant pressure of 1 atmosphere by five resin injection nodes at the center of the mesh. The FEA solver is run until the entire mesh is filled with fluid.

The simulations shown in Figure 50 illustrate the importance of accounting for fabric and tow shearing effects at higher angles (above $20^{\circ}$ ). The differences in mold fill times and tow saturation for simulations (i)-(iii) is significant. If full tow impregnation is desired, the appropriate cycle time must be used to ensure part quality. Note in the baseline case as tows are not separately modeled, they will fill at the same rate as the filling in between the fiber tows (single scale filling). Thus the difference in fill times between the baseline and the other cases quantify additional time needed to fill the fiber tows. This can be seen by the slightly longer fill times 
required to fill the undeformed preforms with the addition of the 1D tow elements. This is logical, as the porous volume being added to the model has permeability which is less than an order of magnitude than that of the bulk preform. Also, when the shear angle approaches $35^{\circ}$, the bulk fabric permeability approaches the constant tow permeability modeled in simulation (ii). As this happens, the fiber tow elements begin to fill at the same rate as the bulk 3D elements. This explains why the fill times for simulation (i) and (ii) converge for the case of $35^{\circ}$ fabric shear. As expected, accounting for shearing of the tows reduces their permeability requiring significantly larger fill times than the simulation in which the tow shearing is ignored as the angle of shear increases.

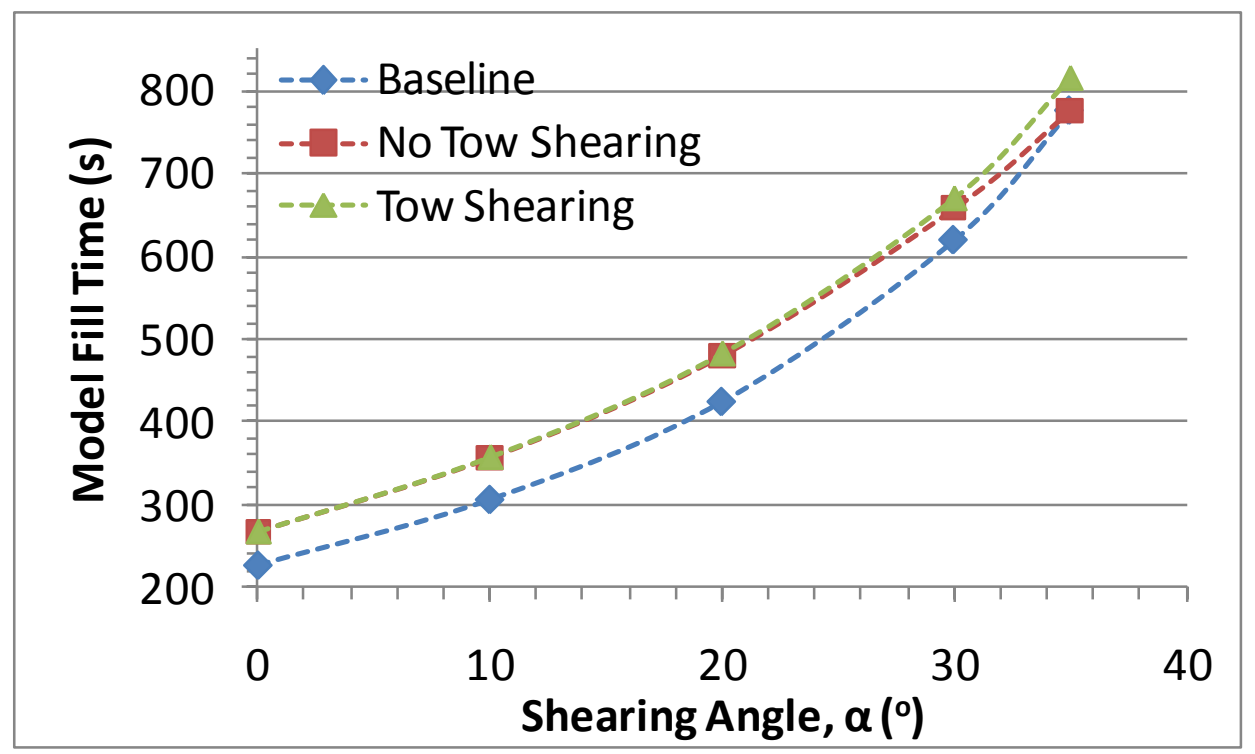

Figure 50: Influence of shear angle on LCM simulations. Baseline case assumes that tows and intertow regions fill at the same rate. No tow shearing case assumes that tows fill at much slower rate according to Figure 4. Tow shearing case results in even lower permeability needing extra time to completely fill the sheared tows. 
It is also useful to know the pressure profile and saturation index for mesh nodes at different times during the simulation. Similar numerical experiments were conducted with the purpose of observing how the one dimensional tow elements fill as a function of time for different simulation schemes. Table 11 and Figure 51 show the difference in preform voidage (fraction of unfilled preform) for simulations (i)-(iii) with a constant induced shear angle of 35 degrees. It can be seen that simulation (ii) follows the same filling profile as that of (i), but with a slight shift due to the added resistance of the 1D sink elements. For case (iii), in which the effect of fabric shear on tow characteristics is accounted for, the 1D tow elements fill at a much slower rate due to the decreased permeability. This demonstrates how taking into account the reduction in tow permeability influences tow filling. The reduced tow permeability greatly affects the saturation rate, requiring much longer time to fill the tows. 
Table 11: A comparison of the amount of unfilled preform versus time for the three simulation types of interest. The amount of unfilled volume is recorded every 100 seconds for each simulation to demonstrate how the simulation that accounts for the reduction in tow permeability (iii) fills slower than its counterparts.

\begin{tabular}{|c|c|c|c|}
\hline Time (s) & Baseline & No Tow Shearing & Tow Shearing \\
\hline 0 & $100.00 \%$ & $100.00 \%$ & $100.00 \%$ \\
100 & $81.00 \%$ & $81.33 \%$ & $84.24 \%$ \\
200 & $66.34 \%$ & $66.75 \%$ & $70.81 \%$ \\
300 & $52.72 \%$ & $53.15 \%$ & $58.12 \%$ \\
400 & $39.68 \%$ & $40.08 \%$ & $45.13 \%$ \\
500 & $26.49 \%$ & $26.89 \%$ & $31.78 \%$ \\
600 & $13.92 \%$ & $14.12 \%$ & $18.52 \%$ \\
700 & $5.06 \%$ & $5.23 \%$ & $7.48 \%$ \\
800 & $0.00 \%$ & $0.00 \%$ & $0.36 \%$ \\
900 & & & $0.00 \%$ \\
\hline Fill Time (s) & $\mathbf{7 7 6}$ & $\mathbf{7 7 7}$ & $\mathbf{8 1 6}$ \\
\hline
\end{tabular}

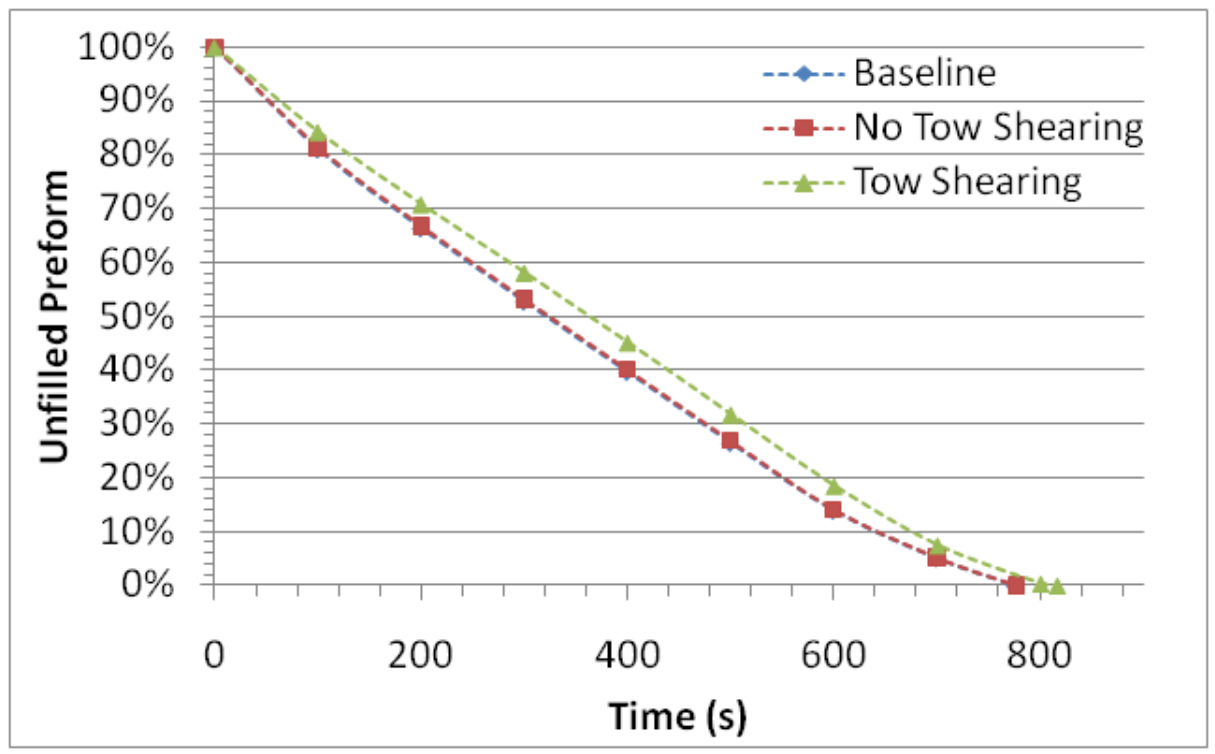

Figure 51: Fraction of unfilled preform as a function of time for simulations when the preform was subjected to a shear angle of $35^{\circ}$ 
The comparison of the progression of tow filling contours for simulations (i), (ii) and (iii) is displayed in Figure 52. The saturation index (value between 0 empty to 1 - full) for the case of 35 degree induced shear is plotted for the each given finite element mesh. The color blue represents the case where the saturation index is zero, or no fluid has impregnated that region of the preform. This then progresses down the color spectrum until the tows are fully saturated, represented by the color red. The region in between can be considered as the partially saturated region. As can be seen by the images in Figure 52(b), the tows saturate faster in case (ii). This simulation finishes filling the tows at approximately 776 seconds. The tows in case (iii) fill much slower when compared with (ii). The reduction in tow permeability slows the tow saturation rate so that full preform saturation requires more than $5 \%$ more time for case (iii) for the constant pressure injection ( $\mathrm{t}_{\mathrm{fill}}=816$ seconds). These simulations are modeling flow through a six inch square preform with four relatively thin plies of fabric. If the size or volume of the preform were to be increased, there may be a much more drastic difference in mold fill times between the two simulations. For the sake of computation time, however, the small yet very fine mesh was desired so that the difference in saturated and unsaturated flow fronts could be tracked. Larger mold volumes will be investigated in a following section and it will be confirmed that the reduction in tow permeability greatly affects mold filling time. This contrast in tow fill times suggests that it is extremely important to correctly model how fabric and tow deformation effect tow saturation in order to ensure part quality. 
(a) Simulation (i)

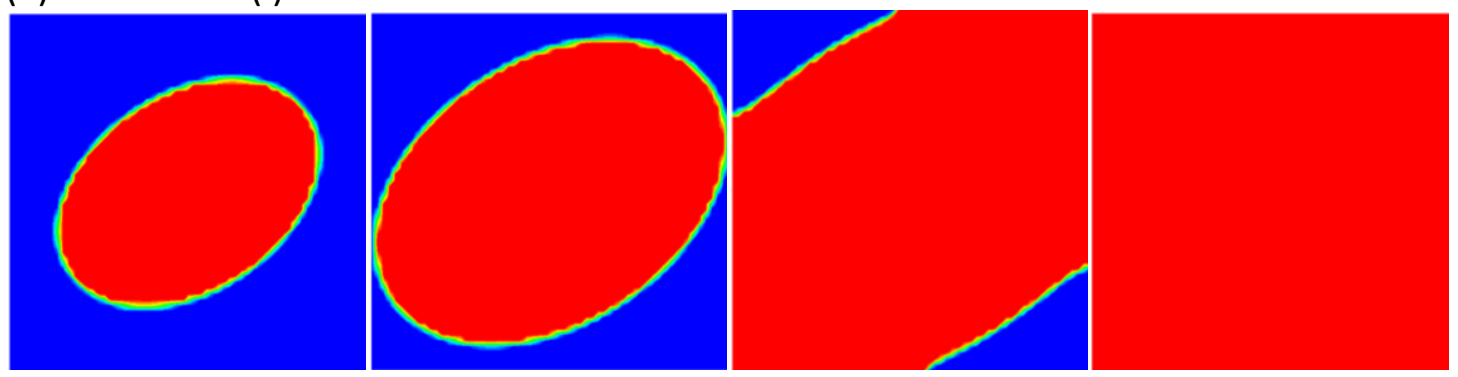

(b) Simulation (ii)

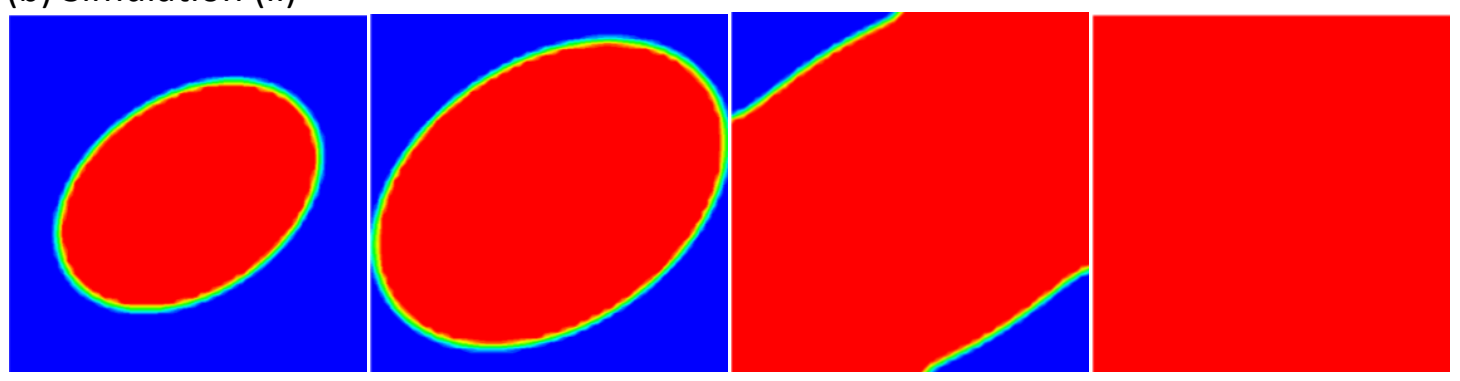

(c) Simulation (iii)



Saturation Index

0

Figure 52: Comparison of tow saturation flow front for simulations (i), (ii) and (iii) to observe the influence of tow deformation of filling 
Much can be learned from these simple simulations. These learning's may then be applied to more complicated geometries and preforms to aid in the prediction of problem areas in processing a specific composite part.

It can be seen that accounting for the mesoscopic deformation within a dual scale fabric is necessary to accurately predict the rate and level of tow saturation in a finite element model. To illustrate this with a practical application, we will look at the resin injection of a hemisphere draped with several fabric layers. Louis and Huber [18] performed a similar simulation, in which the correlation between experimental and numerical flow fronts and fill times were compared. While their experimental data coincided very well with the simulation results, their model did not account for fiber tow saturation. This is where we will look to improve this ability.
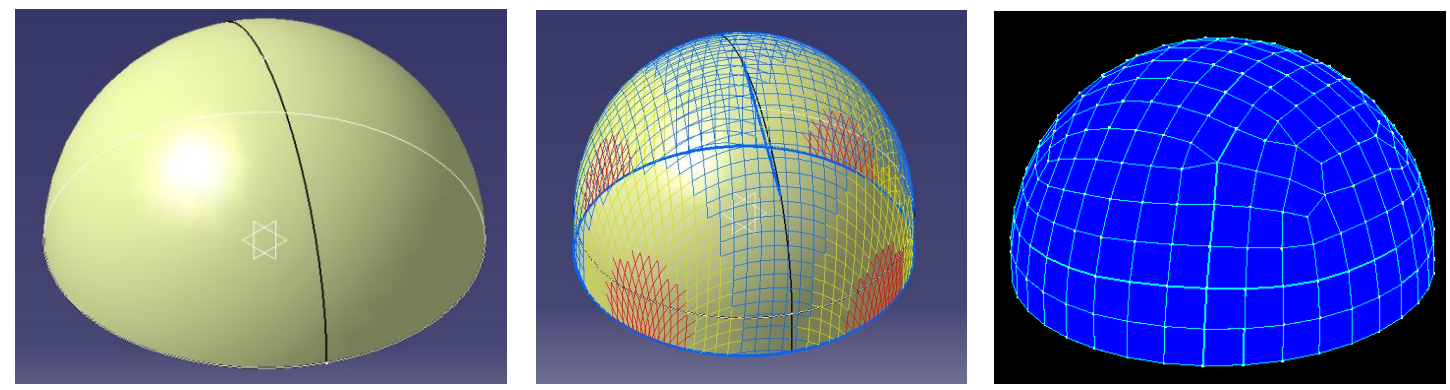

Figure 53: From left to right, a CAD model of tool surface, the draping simulation showing fabric shear zones, and the final 3D mesh in LIMS

A hemisphere the same diameter as used in [18] composed of 2196 nodes and 1794 elements (696 3D bulk elements and 1098 1D tow elements) will serve as our tool surface for the following simulations. A constant pressure injection from six inlet nodes centered on top of the hemisphere, as well as a constant flow rate injection 
simulation will be performed and analyzed. The same simulation conditions were used ( 1 atm., 0.5 Pa-s) for two plies of the characterized fabric with a uniform fabric layup (all in $0^{\circ}$ direction).

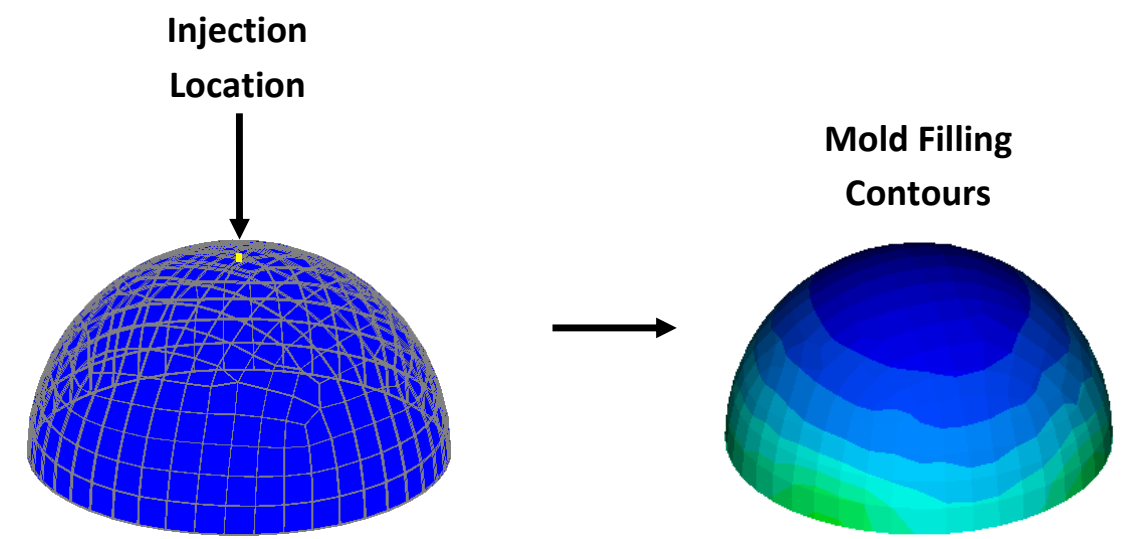

Figure 54: Fluid injection simulation in a hemispherical mold

First, we shall investigate the case of flow driven by a uniform inlet pressure. Four cases will be compared, similar to what was done previously, comparing simulations in which (iii) tow shearing effects are accounted for, (ii) the case for which they are not, as well as new (i) simulations in which no fabric or tow shearing effects (no fiber volume fraction and permeability evolution) are modeled, and (iv), the case when fabric shear is limited to 35 degrees. The reason behind investigating the case when fabric shear is limited to $35^{\circ}$ is because of assumptions that were previously made; in that shear in excess of this magnitude will result in fiber tow slippage. It was this reasoning that influenced the decision to experimentally investigate the effect on dual scale flow for this initial range of fabric shear. Simulation (iv) will represent a hypothetical case which aims to predict what would 
happen in a real manufacturing setting, in which the fiber tows in the sheared fabric are allowed to slip relative to one another. If the local element shear angle is defined as greater than $35^{\circ}$, it is assumed the fabric and fiber tows will slip and the element shear angle $\alpha$ is held to $35^{\circ}$ for both the 3D bulk and 1D (fiber tow) elements. As was discussed previously, for a more accurate description of fabric deformation a more precise draping simulation is needed which accounts for the appropriate resistance to shear, friction, and tow slippage.

It must be noted, however, that the hemisphere models that do account for varying preform FVF but without limitations on the magnitude of possible fabric shear have small areas in which the local elements have negative predicted porous volume, i.e.:

$$
v_{f, \text { shear }}=f_{n}\left(\frac{v_{f, o}}{\cos \alpha}\right)>1
$$

To conserve the porous volume of the model, this negative volume was redistributed into the surrounding 1D tow elements by adjusting the 1D element cross-sectional area. In models with unrealistic local fabric shear angles, the simulation may over predict the time it may take to fully saturate some areas because the porous macro volume has been redistributed to less permeable 1D tow elements. For the case of the hemisphere, there are several small areas in which this happens with shear angles just over the limit, and we assume that the model is still accurate for this geometry. 
Draping Simulation

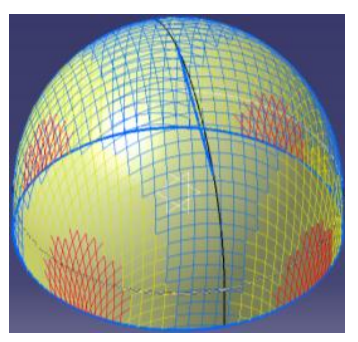

$0^{\circ}<\alpha<24^{\circ}$

$24^{\circ}<\alpha<48^{\circ}$

$\alpha>48^{\circ}$
3D element FVF

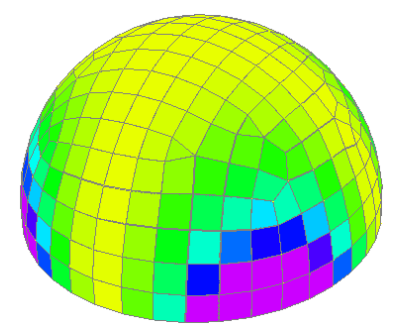

0.70

0.95
Tow Permeability

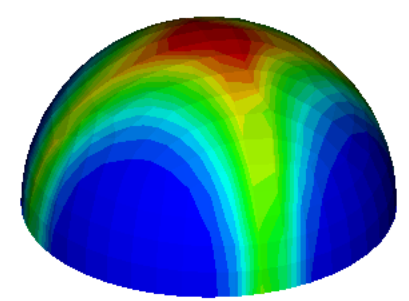

$1 \times 10^{-16} \mathrm{~m}^{2} \quad 1 \times 10^{-12} \mathrm{~m}^{2}$

Figure 55: The draping simulation performed on the hemisphere mold surface, and the resulting $3 D$ element $F V F$ and tow element permeabilities

As expected, there is no real significant difference in flow fronts or tow element saturation for the beginning part of the simulations (ii), (iii), and (iv) as were described above. As can be seen by the center image of Figure 53, the regions around the top of the hemisphere experience little to no shearing. Viewing Figure 56a, the model that does not account for any fabric shear effects has a more uniform flow front around the hemisphere diameter. For simulations (iii) and (iv), a greater gap between the saturated (red) and bulk flows can be seen as expected, due to the decreased tow permeability from the high local shearing. As the flow progresses towards the equator, higher degrees of local fabric shear will be experienced (as high as $50^{\circ}$ ). 
(a) No fabric shearing effects
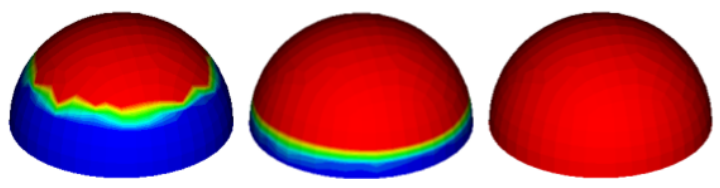

(b) Simulation (ii)
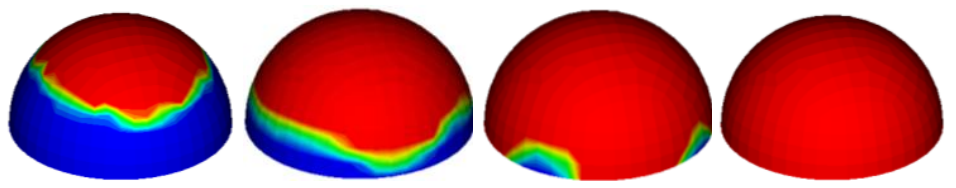

(c) Simulation (iii)
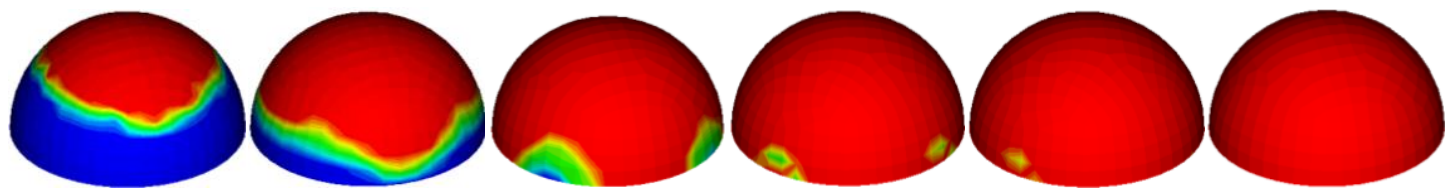

(d) Limited Fabric Shear
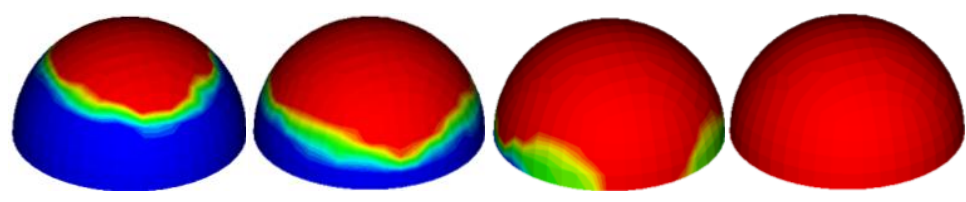

\section{Saturation Index}

0

Figure 56: Comparison of simulation techniques and their corresponding flow fluid flow fronts. Simulations shown in (a) represent the case when no fabric shearing effects are accounted for in the model, $(b)$ and (c) are the simulation types as described earlier, and (d) represents the case in which it is assumed that fabric shear in excess of $35^{\circ}$ will cause fiber tow slippage, and the corresponding elements are held to properties defined by the $35^{\circ}$ maximum shear values.

Upon visual inspection, it appears that the bulk flow fronts for simulation (ii), (iii), and (iv) are comparable. Once the flow front reaches areas within the model 
that have higher fabric shear angles, the flow front progression diverge from one another. The resin flow in the model accounting for tow deformation takes the path of least resistance and flows in the 3D elements (fabric macro-pores) due to their higher permeability, filling those mesh elements faster. Once all of the inter-tow channels are filled, the resin must saturate the less permeable tow elements. In the case of the hemisphere draping, there are areas of high fabric shear approximately $50^{\circ}$ that greatly reduce the permeability of the $1 \mathrm{D}$ elements in those locations.

Table 12: Percentage of unfilled hemispherical preform comparison for the four different model simulations

\begin{tabular}{|c|c|c|c|c|}
\hline Time (s) & No Fabric Shearing & No Tow Shearing & Tow Shearing & Limited Fabric Shear \\
\hline $\mathbf{0}$ & $100.00 \%$ & $100.00 \%$ & $100.00 \%$ & $100.00 \%$ \\
$\mathbf{3 0 0}$ & $56.32 \%$ & $59.61 \%$ & $60.10 \%$ & $60.11 \%$ \\
$\mathbf{6 0 0}$ & $23.28 \%$ & $27.29 \%$ & $28.24 \%$ & $26.52 \%$ \\
$\mathbf{9 0 0}$ & $0.00 \%$ & $5.34 \%$ & $6.19 \%$ & $4.44 \%$ \\
$\mathbf{1 2 0 0}$ & & $0.00 \%$ & $1.16 \%$ & $0.00 \%$ \\
1500 & & & $0.29 \%$ & \\
1700 & & & $0.19 \%$ & \\
1800 & & & $0.08 \%$ & \\
1900 & & & $0.05 \%$ & \\
$\mathbf{2 0 0 0}$ & & $\mathbf{1 1 8 0}$ & $\mathbf{1 9 8 1}$ & \\
\hline Fill Time (s) & $\mathbf{7 9 8}$ & & & 1020 \\
\hline
\end{tabular}

The model in which shearing effects are considered in the 1D elements fills slower than the model with constant tow properties. Simulation (iii) requires an approximately $70 \%$ longer fill time than the previously developed model (ii) for the 
preform. This increased mold fill time is due to the extremely low rate at which the fiber tows fill in the areas of high mold shear. Again, looking at Figure 56c, there is a small region along the equator for times greater than 900 seconds that requires an additional 1000 seconds to fully saturate when compared to simulation (ii). Table 12 above lists the percentage of the filled mold for each simulation given each measured time step. The last $1.5 \%$ of filling for simulation (iii) requires approximately 800 seconds, which is nearly forty percent of the total mold fill time (1981 seconds). This may be slightly conservative as we do not account for capillary effects which will become important when the flow becomes very slow so in practical situations one can expect the tows to fill faster due to capillary effects which have not been accounted for in this analysis. Simulation (iv), representing the case accounting for a maximum amount of fabric shear, follows the same filling profile as cases (ii) and (iii) for the initial part of mold filling as one would expect. The major difference is seen near the end of the mold filling simulation in which fabric shear angles greater than $35^{\circ}$ are witnessed. With fabric shear limited, the bulk and tow element permeabilities are higher than in case (iii), explaining why the filling profiles deviate from one another, and simulation (iv) fills much faster. Also, Simulation (ii) accounts for the increased fabric shear, and the resulting decrease in fabric permeability in the 3D bulk elements. This reduction in permeability for the elements in which local shear exceeds $35^{\circ}$ results in a longer fill time for simulation (ii) compared to (iv). 


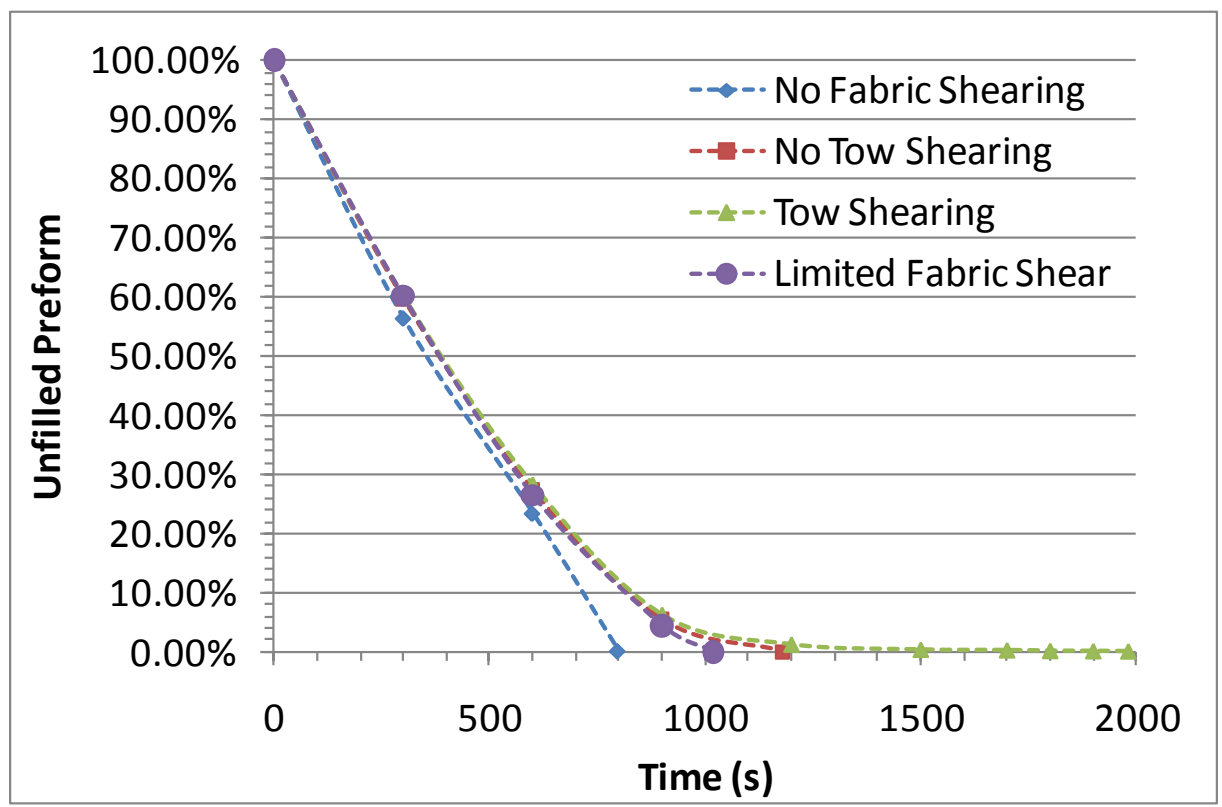

Figure 57: Comparison of the total hemispherical preform fill fraction versus time for each model simulation

Note that usually tow filling cannot be observed by visualization, one has to look at micrographs to observe microvoids inside the tows. Even ultrasonics cannot detect microvoids less than a micron in diameter. Hence most experimental results focus on the filling that does not usually confirm tow filling. Hence our model will allow one to estimate the extra time it would take to completely fill sheared tows.

Some injection molding processes are driven by constant flow rates, produced by screw driven machines. This makes it worthwhile to now look at how accounting for shear dependent tow deformation affect the pressure constraint of a constant flow rate injection. Also, this will allow us to confirm that it is indeed the low permeability tow elements that are responsible for the increased mold fill times. For these simulations, all conditions and models remain the same, except for the 0.1 
$\mathrm{cm}^{3} / \mathrm{s}$ flow rate being used to drive the fluid flow. As expected, the model fill times are the same for the four cases because the control volume has been balanced and is the same. What is drastically different in the four cases is the inlet pressure profiles over the last few seconds of the simulation.

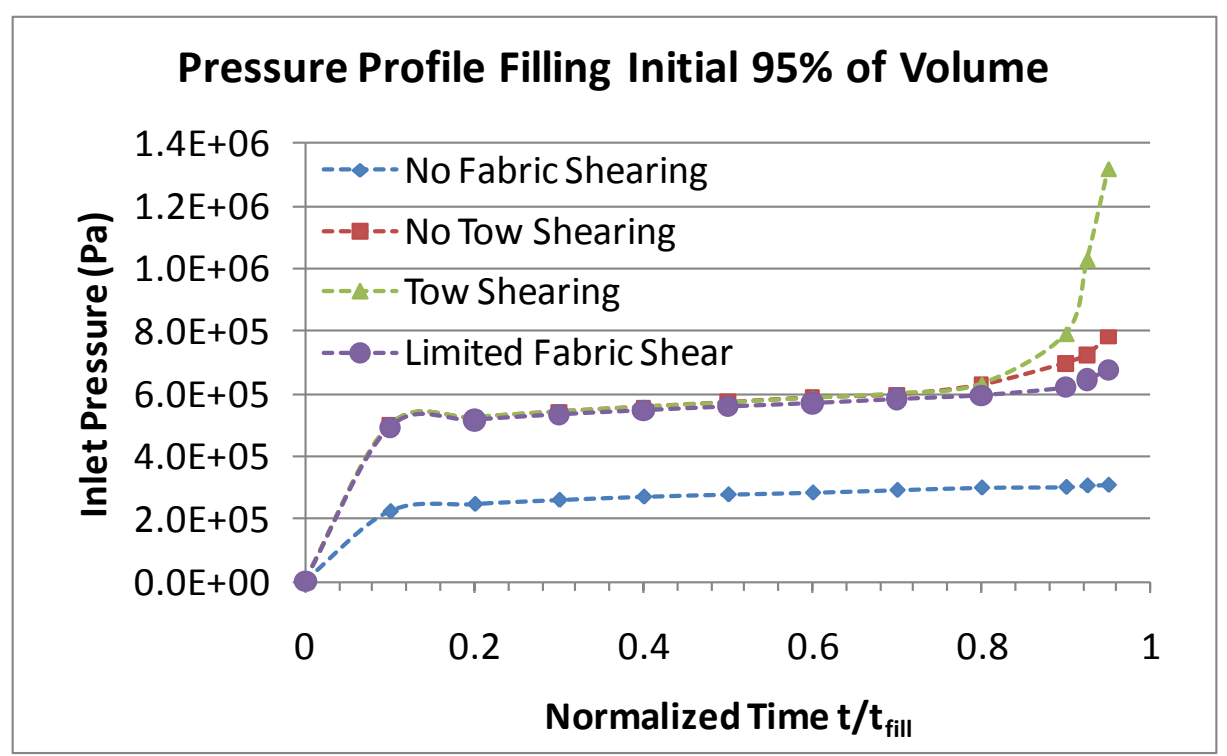

Figure 58: Inlet pressure profile comparison to fill the first $95 \%$ of control volume 


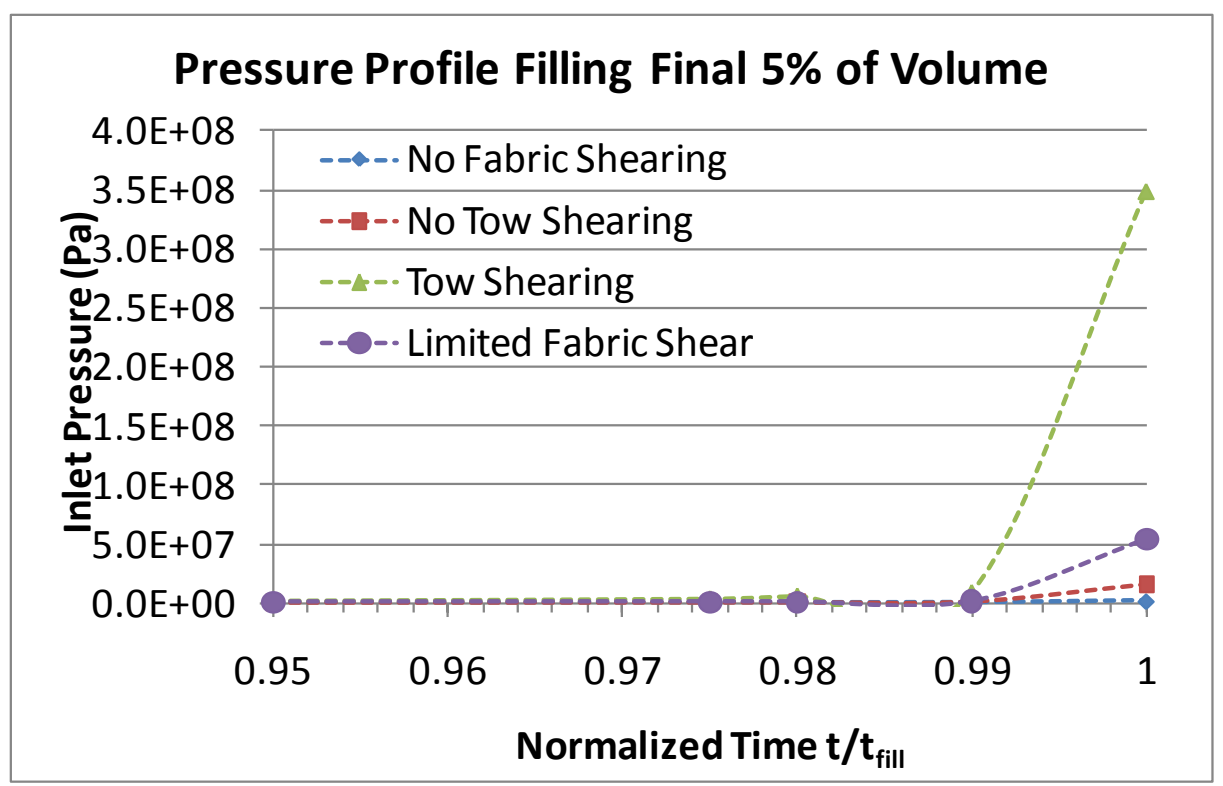

Figure 59: The drastic increase in pressure required to drive the flow for the last 1\% of $C V$ for the case when tow shearing is present

The pressure profiles over the first $80 \%$ of control volume filling (Figure 58) are nearly identical. There are slight discrepancies between the inlet pressure profiles for the next $18 \%$, but a radical difference does not occur again until filling the final $1 \%$ of the model, when a pressure more than an order of magnitude greater is necessary to fully saturate the $1 \mathrm{D}$ tow elements in the high shear zones of simulation (iii). Even the case in which the magnitude of fabric shear is limited (assuming tow slippage) requires significantly higher inlet pressures to drive the fluid flow to fully saturate the final areas of the mold. This confirms that the tow elements in high shear zones have such low permeability, that it requires either very high pressures, or longer cycle times to fully saturate these areas. If these requirements are not met during part processing, there will very likely be dry spots in the areas of interest. 
Another simple shape of interest which contains dual curvature is that of a cone, capped with a hemispherical dome. As was investigated in [43], a six inch tall cone with a one inch radius hemispherical cap and angle of $21^{\circ}$ was used as the tool surface for the draping simulation performed in FiberSIM. The mesh was composed of 9570 nodes, and 8497 elements (3712 3D bulk elements, 4785 1D tow elements). Once again, a constant pressure injection via ten nodes at the vertex of the cone's cap ( 1 atmosphere, fluid viscosity of $0.5 \mathrm{~Pa}-\mathrm{s}$ ) was performed for the four model types simulating flow through four plies of the characterized fabric.

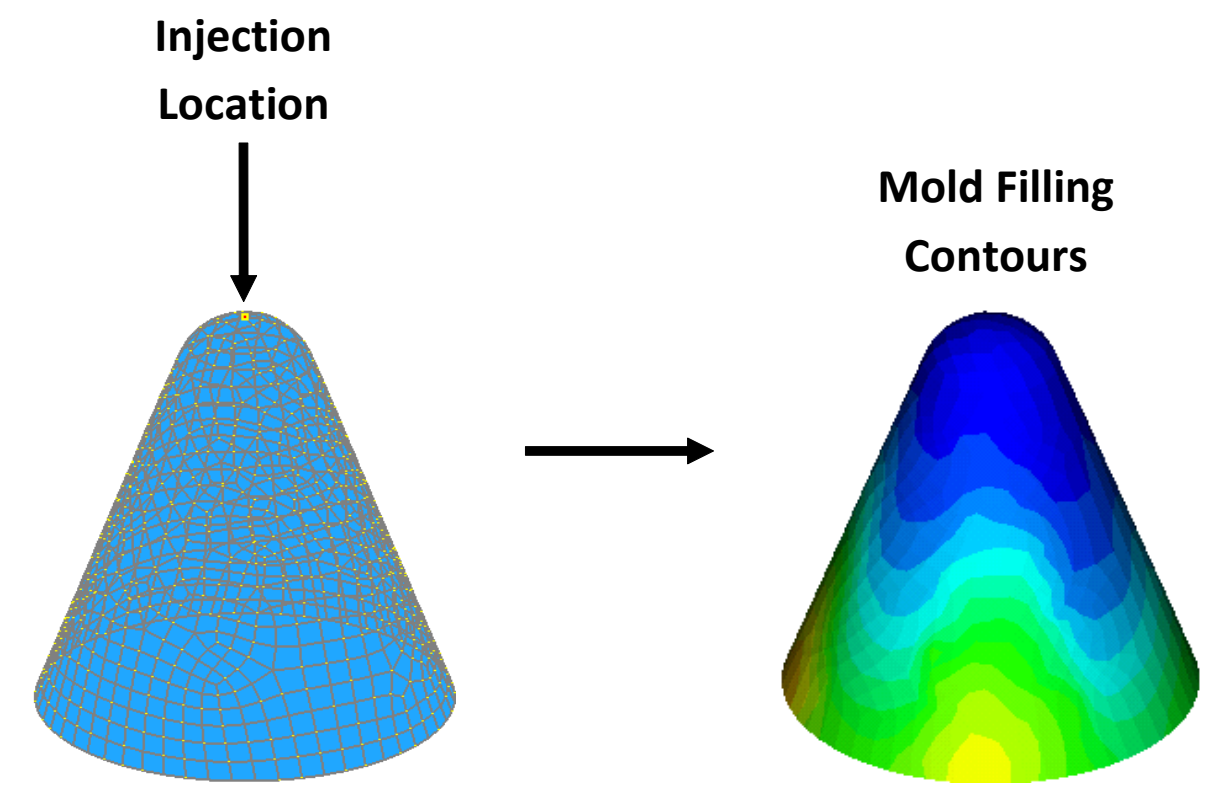

Figure 60: Finite element flow simulation of a conical preform

The cone is of interest because the fishnet draping simulation performed by FiberSIM predicts local fabric shear angles in excess of $60^{\circ}$ (Figure 61). 
This shape is of particular interest because unlike the case of the hemisphere in which there were only several small areas of extreme shear, the cone exhibits these larger shear angles over a much greater percentage of the mold surface area. This extreme fabric shear results in a large amount of the porous volume being redistributed from the $3 \mathrm{D}$ macro elements to the $1 \mathrm{D}$ fiber tow elements.

Draping Simulation

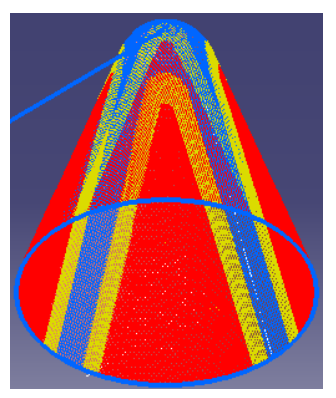

$0^{\circ}<\alpha<24^{\circ}$

$24^{\circ}<\alpha<48^{\circ}$

$\alpha>48^{\circ}$

\section{D element FVF}

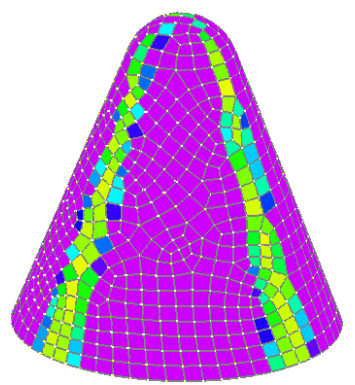

0.70

0.95

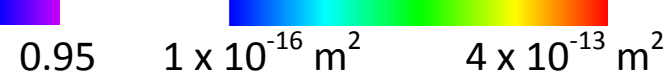

Tow Permeability

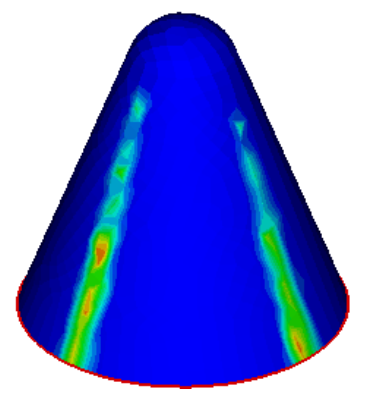

$1 \times 10^{-16} \mathrm{~m}^{2} \quad 4 \times 10^{-13} \mathrm{~m}^{2}$

Figure 61: From left to right: FiberSIM draping simulation over the cone surface, the macro element $F V F$, and the resulting tow element permeabilities

Figure 62 below compares the mold filling of for when fabric and tow shearing effects are and are not accounted for. Once again, the case for which no fabric shearing is modeled fills uniformly and more quickly than its two counterparts. In fact, this model which does not account for the change in fabric permeability fills four times faster (831 seconds) than simulation (ii) (3315 seconds) which does account for fabric shearing effects with a constant tow sink term. Simulation (ii) displays the star shaped flow front that one would expect from the given geometry. 
(a) No Fabric shearing effects

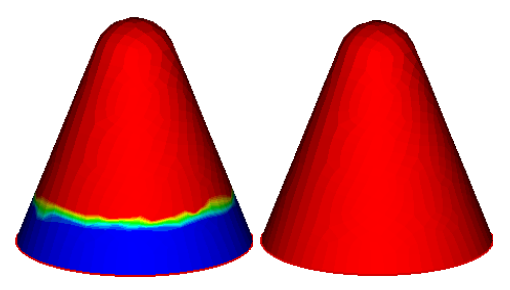

(b) Simulation (ii)

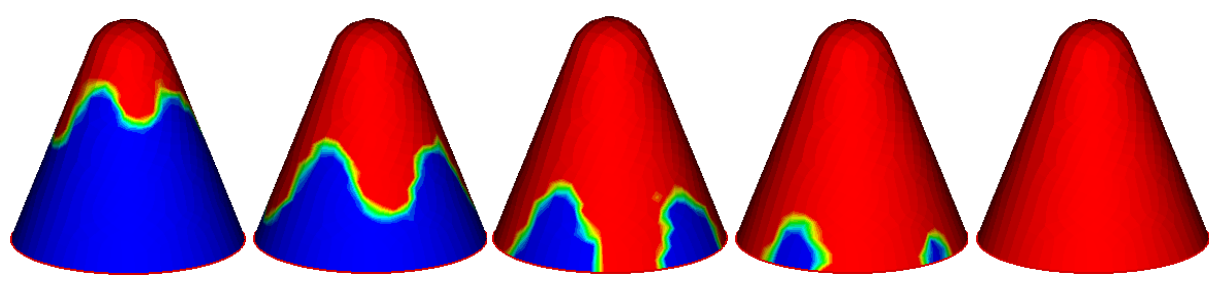

(c) Simulation (iii)
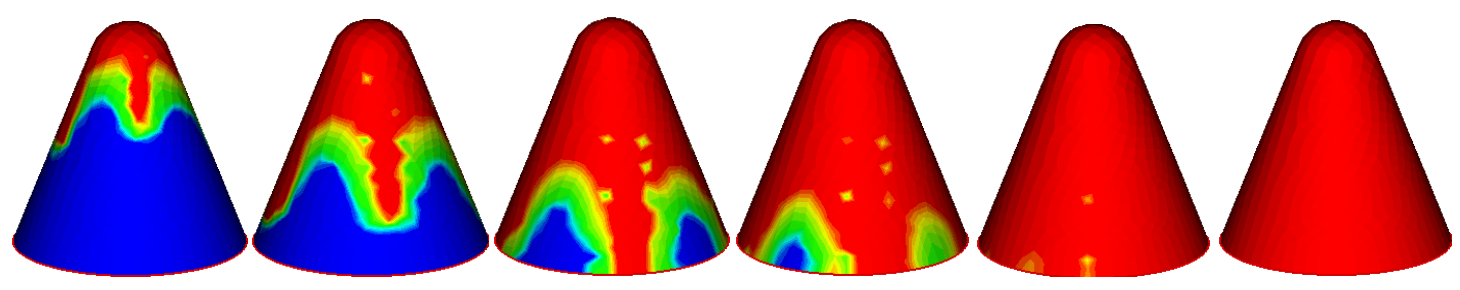

(d) Limited Fabric Shear
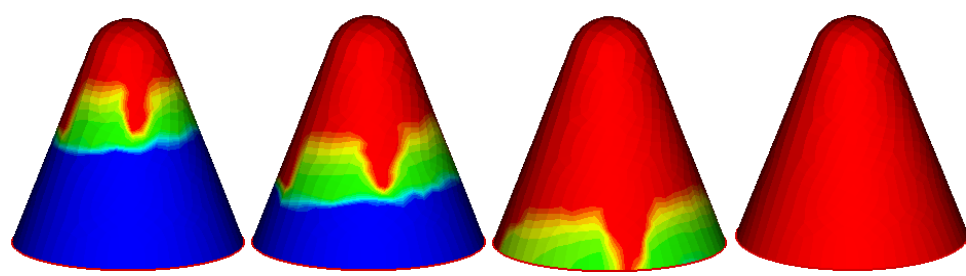

\section{Saturation Index}

0

Figure 62: Comparison of fluid flow fronts through the cone preform for the three different model types 
Once again, the bulk flow fronts are very comparable for simulations (ii) and (iii). As was seen before with the case of the hemisphere, when the fluid flow reaches areas with very low tow permeability (Figure 61), the flow fills the 3D bulk elements faster, taking the least resistive path, and then fills the less permeable tow elements. This is much more evident in the case of the cone than the hemisphere, because the tow permeability is even further reduced by the larger fabric shear angles.

Table 13: Percentage of unfilled preform comparison for the four different conical model simulations

\begin{tabular}{|c|c|c|c|c|}
\hline Time (s) & No Fabric Shear & No Tow Shearing & Tow Shearing & Limited Shear \\
\hline 0 & $100.00 \%$ & $100.00 \%$ & $100.00 \%$ & $100.00 \%$ \\
500 & $32.33 \%$ & $73.78 \%$ & $75.66 \%$ & $73.03 \%$ \\
1000 & $0.00 \%$ & $56.21 \%$ & $58.38 \%$ & $51.86 \%$ \\
1500 & $100.00 \%$ & $39.95 \%$ & $42.22 \%$ & $32.30 \%$ \\
2000 & & $25.75 \%$ & $28.11 \%$ & $12.99 \%$ \\
2500 & & $13.94 \%$ & $15.89 \%$ & $0.00 \%$ \\
3000 & & $5.07 \%$ & $6.81 \%$ & \\
3500 & & $0.00 \%$ & $1.32 \%$ & \\
4000 & & & $0.01 \%$ & \\
\hline Fill Time (s) & 821 & 3315 & 4016 & 2284 \\
\hline
\end{tabular}

The model which accounts for the effects of fabric shear on mold filling at both the macro and micro levels, simulation (iii), takes longer to completely saturate the preform as expected (4016 seconds). The lag between the saturated and unsaturated flow front is greatly increased as is anticipated by the experimental observations due to the decreased tow permeability. For the filling of this mold 
geometry, the new proposed model suggests that under the given constant inlet pressure conditions, one must allow an additional 700 seconds for the fiber tows in the high shear areas new the bottom of the cone to fully saturate. If full tow saturation is desired, this additional time must be allowed for full fiber impregnation.

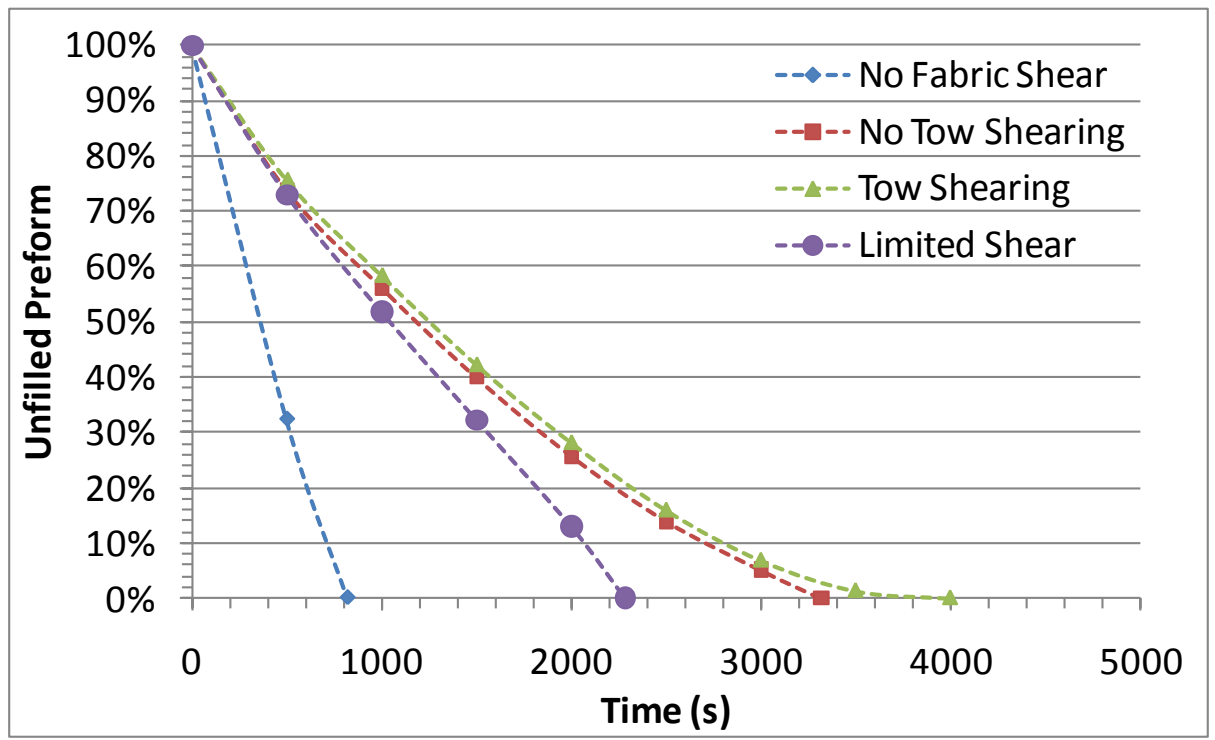

Figure 63: Comparison of the total preform fill fraction versus time for each conical model simulation

Simulation (iv) makes the assumption that a draped fabric will not shear beyond 35 degrees. As discussed previously, the cone is an interesting geometry to test this assumption as the majority of the mold surface experiences shear angles greater than $35^{\circ}$. This is the reason behind the more uniform flow front than the star shaped one seen by (ii), (iii), and in [43]. These results suggest that for this type of geometry with significant fabric shear occurring during draping, a much more accurate 
draping model and the corresponding fabric characterization is needed to successfully predict the dual scale filling of this mold. 


\section{Chapter 5}

\section{CONCLUSIONS, CONTRIBUTIONS AND FUTURE WORK}

It has been shown that fabric shear induced by the draping of a fabric preform over a curved mold surface does indeed affect the dual scale nature of these fibrous reinforcements. Simplified experiments were designed and conducted to fully characterize the dual scale permeability of a specific textile fabric. This information was then used as input, along with a draping simulation, as necessary information to generate a numerical model capable of prediction dual scale flow, specifically tow saturation, in closed mold LCM processes. It was found that the fiber tow permeability can change drastically when subject to shear and influence the mold filling and hence the micro voids within a fiber tow

With the appropriate physics and conversion tool established, onedimensional tow elements were added to an existing 2D or 3D mesh to simulate tow saturation. This ability, coupled with a draping simulation, would then allow an engineer to investigate the effects of draping a fabric over a complex mold in different regions. The comparison of the simulation results above leads us to believe that it is indeed worth investigating how fabric shear effects tow saturation for engineered fabric reinforcements.

The numerical simulations performed herein show that for increased fabric shear angles, it may be necessary to account for associated effects on fiber bundle geometry. This change in local mesoscopic structure may change flow characteristics 
throughout an entire part, so it is necessary to be able to accurately model such phenomenon. With an increased resistance to flow in certain mold regions due to shear, flow front progression may change, and so may the optimum placement of injection gates and resin vents. Moreover, in areas of high shear, a large increase in cycle time may be needed for full tow impregnation. Experimental data paired with this simulation can greatly reduce wasted time and resources in composites design and manufacturing processes.

As was discussed in Chapter 2.1, previously developed numerical models are capable of predicting idealized bulk fluid flow through a preform with a good deal of accuracy. The effect of fabric shear due to draping on the bulk properties of the preform have been studied and successfully integrated into numerical flow simulations. Elementary work has also been completed in modeling the dual scale nature of these technical fabrics by adding a constant sink term to account for tow saturation.

The original work described within this thesis has been aimed at further improving the capability of composites manufacturing predictive models by incorporating the effect of shear deformation on dual scale flow, specifically tow saturation. To start, experiments were performed to determine how fabric shear influenced micro resin flow and tow impregnation in a simplified picture frame design. It had been shown by prior investigators that the fiber tow geometry changes on the filament level when loaded by shear fabric deformation. This led to the belief that the decreased tow porosity would result in lower tow permeability, changing the fiber bundle saturation rate. This was confirmed by the experimental observations. With the increase of induced fabric shear angle by the experimental setup, it was seen 
that the difference between the quicker, unsaturated or bulk flow and the slower saturated region grew larger. The saturation lag length was determined as a function of fabric shear and appeared to be exponential in nature.

The tow saturation lag lengths were used as a basis for tow permeability characterization as a function of fabric shear. Finite element models were developed with the presence of one-dimensional tow elements to simulate the corresponding experiments. The one-dimensional tow element permeability was adjusted until the saturated flow lag length agreed with experimental data for each discrete shear angle. With the effective tow permeabilities found and the bulk fabric properties known, the specific engineered fabric had been fully characterized for the simulation of the effect of shear deformation on dual scale flow. This information was then used in all future simulation work, investigating the importance of accounting for the varying tow permeability in mold filling.

Several assumptions were made when modeling the shear deformation and the physics surrounding tow saturation. The simplifications were introduced because of limitations in the numerical modeling software as well as time constraints. Future work on the subject should more thoroughly investigate how the microstructure of the fibrous reinforcement changes with shear and the draping of a fabric.

As was discussed in Chapter 2, a constant characteristic flow length was chosen for the length of the one-dimensional tow elements. This characteristic length describes the length that the pressure driven flow must travel to fully impregnate the fiber tows. In our model, the length was designated to be half of the thickness of a fiber tow. This model then does not account for geometric changes in tow geometry, 
whether positive or negative, induced by fabric shear, crimp, or other loading, nor does it account for areas of tow overlap. This work could be improved if one was to more precisely model how tow geometry and porosity change as a function of shear and how tow overlap effects the characteristic flow length. This information could then lead to a more accurate prediction of tow permeability, as opposed to the characterization of the effective one-dimensional element permeability as was performed in this thesis.

Additional areas for improvement in the numerical model are to account for capillary flow effects and trapped air within the fiber tows. The current finite element software is the limiting factor in this case. Also, the one-dimensional tow elements can only be prescribed one tow permeability value. Of course, typical fiber tows will have two distinct permeabilities, in the longitudinal and transverse directions. If the tow elements can be improved to account for directional permeabilities, this will only aid in the accuracy of the numerical model.

Finally, a proper convergence study should be conducted for the cases of the resin flow through the hemispherical and conical preforms. One must refine the meshes significantly, and model all process parameters consistently and accurately to determine whether the results converge. Although it would be difficult to verify, it would be beneficial to validate the model tow saturation results experimentally. Because of the amount of time this action would require, it was not completed at this time. 


\section{REFERENCES}

[1] Simacek, P., \& Advani, S. G. (2004). Desirable features in mold filling simulations for liquid composite molding processes. Polymer Composites, 25(4), 355-367.

[2] Joubaud, L., F. Trochu, and J. Le Corvec. "Analysis of Resin Flow Under Flexible Cover in Vacuum Assisted Resin Infusion (VARI)." Journal of Advanced Materials 37.3 (2005): 3-10.

[3] Lawrence, Jeffrey M., et al. "Simulation and Validation of Resin Flow during Manufacturing of Composite Panels Containing Embedded Impermeable Inserts with the VARTM Process." Polymer Composites 28.4 (2007): 442-50.

[4] Lawrence, Jeffrey M., Patrice Hughes, and Suresh G. Advani. "Experimental Validation of Dependence Map Based Control in Liquid Composite Molding." Journal of Composite Materials 40.13 (2006): 1137-62.

[5] Tan, Hua, and Krishna M. Pillai. "Fast Liquid Composite Molding Simulation of Unsaturated Flow in Dual-Scale Fiber Mats using the Imbibition Characteristics of a Fabric-Based Unit Cell." Polymer Composites 31.10 (2010): 1790-807.

[6] Lawrence, J. M., et al. "An Approach to Couple Mold Design and on-Line Control to Manufacture Complex Composite Parts by Resin Transfer Molding." Composites Part A-Applied Science and Manufacturing 33.7 (2002): 981-90.

[7] Johnson, R. J., and R. Pitchumani. "Active Control of Reactive Resin Flow in a Vacuum Assisted Resin Transfer Molding (VARTM) Process." Journal of Composite Materials 42.12 (2008): 1205-29.

[8] Chohra, Mourad, et al. "Modeling of Filtration through Multiple Layers of Dual Scale Fibrous Porous Media." Polymer Composites 27.5 (2006): 570-81.

[9] Reprinted from Composites Part A: Applied Science and Manufacturing, 37, Nina Kuentzer, Pavel Simacek, Suresh G. Advani, Shawn Walsh, Permeability characterization of dual scale fibrous porous media, 2057-2068, 2006, with permission from Elsevier. 
[10] Zhou, F., Kuentzer, N., Simacek, P., Advani, S. G., \& Walsh, S. (2006). Analytic characterization of the permeability of dual-scale fibrous porous media. Composites Science and Technology, 66(15), 2795-2803.

[11] Kuentzer, N., Simacek, P., Advani, S. G., \& Walsh, S. (2006). Permeability characterization of dual scale fibrous porous media. Composites Part AApplied Science and Manufacturing, 37(11), 2057-2068.

[12] Ngo, N., \& Tamma, K. K. (2001). Microscale permeability predictions of porous fibrous media. International Journal of Heat and Mass Transfer, 44(16), 3135-3145.

[13] Verleye, B., Croce, R., Griebel, M., Klitz, M., Lomov, S. V., Morren, G., et al. (2008). Permeability of textile reinforcements: Simulation, influence of shear and validation. Composites Science and Technology, 68(13), 2804-2810.

[14] Demaria, C., Ruiz, E., \& Trochu, F. (2007). In-plane anisotropic permeability characterization of deformed woven fabrics by unidirectional injection. part I: Experimental results. Polymer Composites, 28(6), 797-811.

[15] Dungan, F. D., Senoguz, M. T., Sastry, A. M., \& Faillaci, D. A. (1999). On the use of permeability in sheared fabrics. Journal of Reinforced Plastics and Composites, 18(5), 472-484.

[16] Heardman, E., Lekakou, C., \& Bader, M. G. (2001). In-plane permeability of sheared fabrics. Composites Part A-Applied Science and Manufacturing, 32(7), 933-940.

[17] Smith, P., Rudd, C. D., \& Long, A. C. (1997). The effect of shear deformation on the processing and mechanical properties of aligned reinforcements. Composites Science and Technology, 57(3), 327-344.

[18] Louis, M., \& Huber, U. (2003). Investigation of shearing effects on the permeability of woven fabrics and implementation into LCM simulation. Composites Science and Technology, 63(14), 2081-2088.

[19] Slade, J., Sozer, E. M., \& Advani, S. G. (2000). Fluid impregnation of deformed preforms. Journal of Reinforced Plastics and Composites, 19(7), 552-568. 
[20] Endruweit, A., \& Ermanni, P. (2004). The in-plane permeability of sheared textiles. Experimental observations and a predictive conversion model. Composites Part A-Applied Science and Manufacturing, 35(4), 439-451.

[21] Demaria, C., Ruiz, E., \& Trochu, F. (2007). In-plane anisotropic permeability characterization of deformed woven fabrics by unidirectional injection. part II: Prediction model and numerical simulations. Polymer Composites, 28(6), 812827.

[22] Badel, P., Vidal-Salle, E., Maire, E., \& Boisse, P. (2008). Simulation and tomography analysis of textile composite reinforcement deformation at the mesoscopic scale. Composites Science and Technology, 68(12), 2433-2440.

[23] Simacek, P., \& Advani, S. G. (1996). Permeability model for a woven fabric. Polymer Composites, 17(6), 887-899.

[24] Grujicic, M., Chittajallu, K. M., \& Walsh, S. (2006). Lattice boltzmann method based computation of the permeability of the orthogonal plain-weave fabric preforms. Journal of Materials Science, 41(23), 7989-8000.

[25] Nordlund, M., \& Lundstrom, T. S. (2005). Numerical study of the local permeability of noncrimp fabrics. Journal of Composite Materials, 39(10), 929-947.

[26] Nabovati, A., Llewellin, E. W., \& Sousa, A. C. M. (2010). Through-thickness permeability prediction of three-dimensional multifilament woven fabrics. Composites Part A-Applied Science and Manufacturing, 41(4), 453-463.

[27] Zhou, Fuping, et al. "Analytic Characterization of the Permeability of DualScale Fibrous Porous Media." Composites Science and Technology 66.15 (2006): 2795-803.

[28] Skelton, J. "Fundamentals of Fabric Shear." Textile Research Journal 46.12 (1976): 862-9.

[29] Reprinted from Polymer Composites, C. Demaria, Edu Ruiz, F. Trochu, InPlane anisotropic permeability characterization of deformed woven fabrics by unidirectional injection. Part I: Experimental results, 797-881, 2007, with permission from John Wiley and Sons.

[30] Gebart, B. R., and P. Lidstrom. "Measurement of in-Plane Permeability of Anisotropic Fiber Reinforcements." Polymer Composites 17.1 (1996): 43-51. 
[31] Bruschke, M. V., and S. G. Advani. "A Finite-Element Control Volume Approach to Mold Filling in Anisotropic Porous-Media." Polymer Composites 11.6 (1990): 398-405.

[32] Reprinted from Composites Part A: Applied Science and Manufacturing, 35, A. Endruweit, P. Ermanni, The in-plane permeability of sheared textiles. Experimental observations and a predictive conversion model, 439-451, 2004, with permission from Elsevier.

[33] Zhou, Fuping, Justin Alms, and Suresh G. Advani. "A Closed Form Solution for Flow in Dual Scale Fibrous Porous Media Under Constant Injection Pressure Conditions." Composites Science and Technology 68.3-4 (2008): 699-708.

[34] Simacek, P., \& Advani, S. G. (2003). A numerical model to predict fiber tow saturation during liquid composite molding. Composites Science and Technology, 63(12), 1725-1736.

[35] Reprinted from Composites Science and Technology, 63, Pavel Simacek, Suresh G. Advani, , A numerical model to predict fiber tow saturation during liquid composite molding, 1725-1736, 2003, with permission from Elsevier.

[36] Schell, J. S. U., Siegrist, M., \& Ermanni, P. (2007). Experimental determination of the transversal and longitudinal fibre bundle permeability. Applied Composite Materials, 14(2), 117-128.

[37] Simacek, P., Neacsu, V., \& Advani, S. G. (2010). A phenomenological model for fiber tow saturation of dual scale fabrics in liquid composite molding. Polymer Composites, 31(11), 1881-1889.

[38] FiberSIM. Computer software. Composite CAD Software. Vers. 2009. VISTAGY. Web. <http://www.vistagy.com/products/fibersim-compositeenvironments.aspx $>$.

[39] ABAQUS/CAE. Computer Software. ABAQUS Finite Element Modeling. Vers. 6.8 Student Edition. SIMULIA. $<$ http://www.simulia.com/academics/purchase.html>.

[40] Martinez, F., Simacek, P., \& Advani, S. G. (2010). "Comparison of modeling approaches to resin flow simulation in layered draped textile preforms." Recent Advances in Textile Composites: Proceedings of the 10th International Conference on Textile Composites, 463-471. 
[41] Reprinted from Composites Part A: Applied Science and Manufacturing, 40, J.M. Lawrence, V. Neacsu, S.G. Advani, Modeling the impact of capillary pressure and air entrapment on fiber tow saturation during resin infusion in LCM, 1053-1064, 2009, with permission from Elsevier.

[42] Reprinted from Polymer Composites, P. Simacek, V. Neacsu, S.G. Advani, A phenomenological model for fiber tow saturation of dual scale fabrics in liquid composite molding, 1881-1889, 2010, with permission from John Wiley and Sons.

[43] Bickerton, S., et al. "Investigation of Draping and its Effects on the Mold Filling Process during Manufacturing of a Compound Curved Composite Part." Composites Part A-Applied Science and Manufacturing 28.9-10 (1997): 801-16. Print. 


\section{Appendix A}

\section{IMAGE PROCESSING OUTPUT TABLE}

Table A.1: Table output by image processing code

\begin{tabular}{|l|c|c|c|c|c|}
\hline \multicolumn{2}{|c|}{} & \multicolumn{2}{c|}{ Saturated Ellipse } & Macro Flow Ellipse \\
\hline Image File Name & $\begin{array}{c}\text { Ellapsed } \\
\text { Time (s) }\end{array}$ & $\begin{array}{l}\text { Semi-Major } \\
\text { Axis (in) }\end{array}$ & $\begin{array}{l}\text { Semi-Minor } \\
\text { Axis (in) }\end{array}$ & $\begin{array}{l}\text { Semi-Major } \\
\text { Axis (in) }\end{array}$ & $\begin{array}{l}\text { Semi-Minor } \\
\text { Axis (in) }\end{array}$ \\
\hline JRW-12_0.png & 0 & 0 & 0 & 0 & 0 \\
\hline JRW-12_60.png & 60 & 1.30 & 0.84 & 2.24 & 1.45 \\
\hline JRW-12_90.png & 90 & 1.39 & 0.86 & 2.51 & 1.52 \\
\hline JRW-12_120.png & 120 & 1.62 & 1.04 & 2.80 & 1.71 \\
\hline. &. &. &. &. &. \\
\hline. &. &. &. &. &. \\
\hline. &. &. &. &. &. \\
\hline JRW-12_900.png & 900 & 4.33 & 2.58 & 4.85 & 2.90 \\
\hline JRW-12_930.png & 930 & 4.22 & 2.54 & 4.76 & 2.84 \\
\hline JRW-12_960.png & 960 & 4.38 & 2.64 & 4.94 & 2.96 \\
\hline
\end{tabular}




\section{Appendix B}

\section{PERMISSION LETTERS}

Reprint Permission for Figure 1.

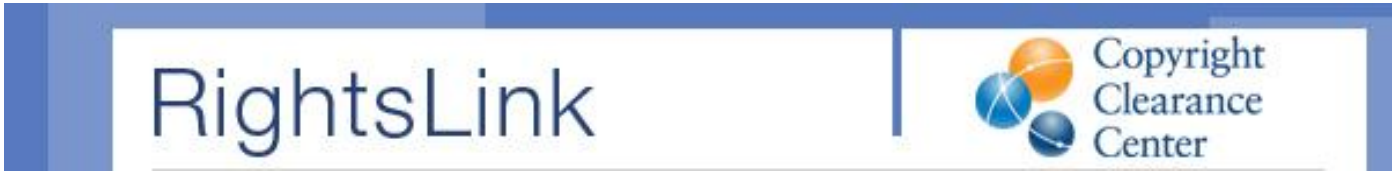

\section{THANK YOU FOR YOUR ORDER!}

Dear Joe Walther,

Thank you for placing your order through Copyright Clearance Center's RightsLink service. Elsevier has partnered with RightsLink to license its content.

Your order details and publisher terms and conditions are available by clicking the link below:

http://s100.copyright.com/CustomerAdmin/PLF.jsp?IID=2011070 1309900751319

\section{Order Details}

Licensee: Joe Walther

License Date: Jul 05, 2011

License Number: 2702691223319

Publication: Composites Part A: Applied Science and Manufacturing

Title: Permeability characterization of dual scale fibrous porous media

Type Of Use: reuse in a thesis/dissertation

Total: 0.00 USD 
Reprint Permission for Figure 2.



\section{THANK YOU FOR YOUR ORDER!}

Dear Joe Walther,

Thank you for placing your order through Copyright Clearance Center's RightsLink service. John Wiley and Sons has partnered with RightsLink to license its content.

Your order details and publisher terms and conditions are available by clicking the link below:

http://s100.copyright.com/CustomerAdmin/PLF.jsp?|ID=20110701309899963194

\section{Order Details}

Licensee: Joe Walther

License Date: Jul 05, 2011

License Number: 2702690435194

Publication: Polymer Composites

Title: In-plane anisotropic permeability characterization of deformed woven fabrics by unidirectional injection. Part I: Experimental results

Type Of Use: Dissertation/Thesis

Total: 0.00 USD 
Reprint Permission for Figure 3.

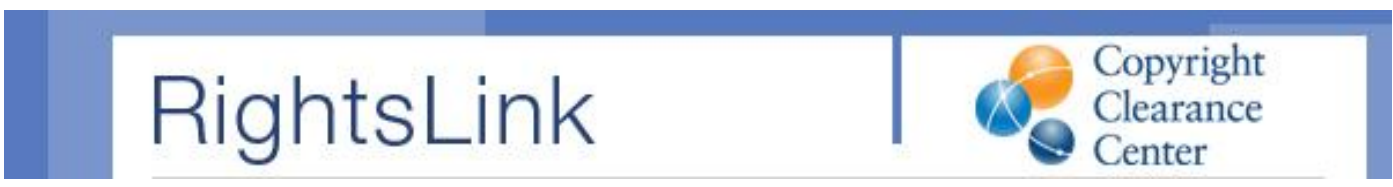

\section{THANK YOU FOR YOUR ORDER!}

Dear Joe Walther,

Thank you for placing your order through Copyright Clearance Center's RightsLink service. Elsevier has partnered with RightsLink to license its content.

Your order details and publisher terms and conditions are available by clicking the link below:

http://s100.copyright.com/CustomerAdmin/PLF.jsp?|ID=2011070 1309900913632

\section{Order Details}

Licensee: Joe Walther

License Date: Jul 05, 2011

License Number: 2702691385632

Publication: Composites Part A: Applied Science and Manufacturing

Title: The in-plane permeability of sheared textiles. Experimental observations and a predictive conversion model

Type Of Use: reuse in a thesis/dissertation

Total: 0.00 USD 
Reprint Permission for Figures 4, 5, and 6.

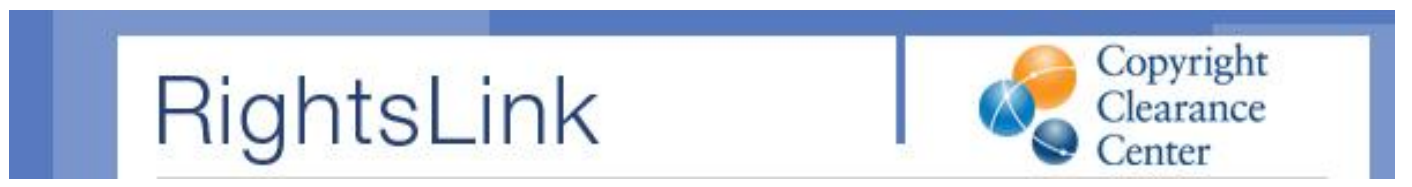

THANK YOU FOR YOUR ORDER!

Dear Joe Walther,

Thank you for placing your order through Copyright Clearance Center's RightsLink service. Elsevier has partnered with RightsLink to license its content.

Your order details and publisher terms and conditions are available by clicking the link below:

http://s100.copyright.com/CustomerAdmin/PLF.jsp?IID=2011070 1309900205 $\underline{178}$

\section{Order Details}

Licensee: Joe Walther

License Date: Jul 05, 2011

License Number: 2702690677178

Publication: Composites Science and Technology

Title: A numerical model to predict fiber tow saturation during liquid composite molding

Type Of Use: reuse in a thesis/dissertation

Total: 0.00 USD 
Reprint Permission for Figure 10.

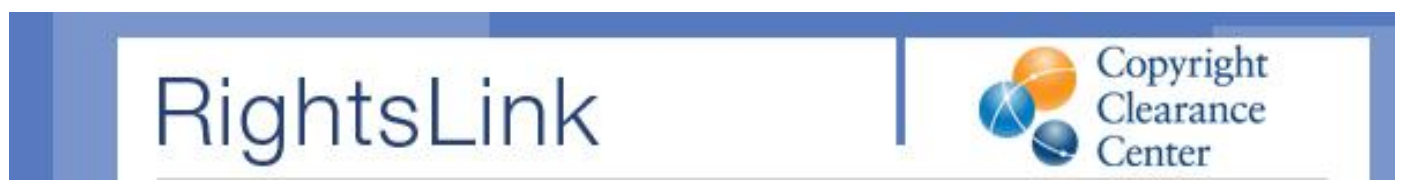

THANK YOU FOR YOUR ORDER!

Dear Joe Walther,

Thank you for placing your order through Copyright Clearance Center's RightsLink service. Elsevier has partnered with RightsLink to license its content.

Your order details and publisher terms and conditions are available by clicking the link below:

http://s100.copyright.com/CustomerAdmin/PLF.jsp?IID=2011070 1309902270991

\section{Order Details}

Licensee: Joe Walther

License Date: Jul 05, 2011

License Number: 2702701230991

Publication: Composites Part A: Applied Science and Manufacturing

Title: Modeling the impact of capillary pressure and air entrapment on fiber tow saturation during resin infusion in LCM

Type Of Use: reuse in a thesis/dissertation

Total: 0.00 USD 
Reprint Permission for Figure 17.

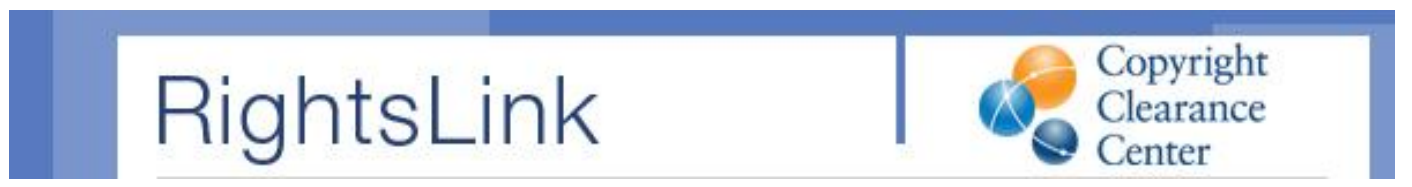

THANK YOU FOR YOUR ORDER!

Dear Joe Walther,

Thank you for placing your order through Copyright Clearance Center's RightsLink service. John Wiley and Sons has partnered with RightsLink to license its content.

Your order details and publisher terms and conditions are available by clicking the link below:

http://s100.copyright.com/CustomerAdmin/PLF.jsp?|ID=2011070 1309899578 $\underline{882}$

\section{Order Details}

Licensee: Joe Walther

License Date: Jul 05, 2011

License Number: 2702690050882

Publication: Polymer Composites

Title: A phenomenological model for fiber tow saturation of dual scale fabrics in liquid composite molding

Type Of Use: Dissertation/Thesis

Total: 0.00 USD 
Appendix C

FIBERSIM TO LIMS CONVERSION TOOL MANUAL 


\section{FIBERSIM 2009 / LIMS}

\section{CONVERSION TOOL}

Version 2.0

USER'S MANUAL

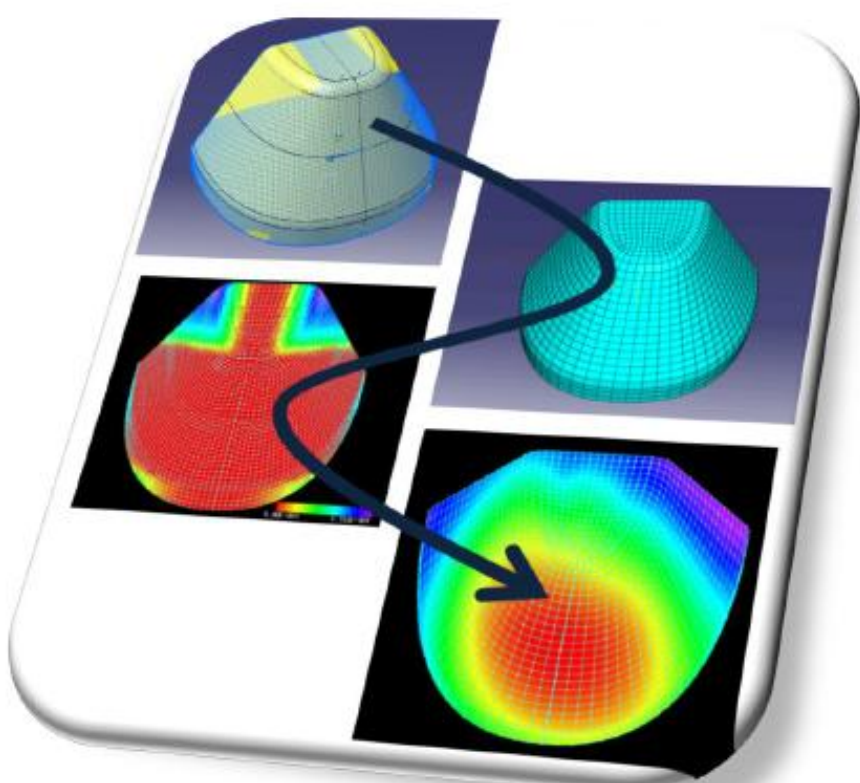




\section{SYSTEM REQUIREMENT:}

- Microsoft eXCEL 2007

- catia v5 R19

- fibersim 2009

- ABAQUS 6.9

- LIMS 5.0.7

\section{SETUP:}

INSTALLATION:

The FIBERSIM 2009 / LIMS Conversion Tool is composed by the following files:

- FibersimLims.xlsx: Excel file with macro.

- Folder "DATA" : ESSENTIAL, FIBERSIM 2009/ LIMS Conversion Toll cannot work without this file.

- Folder "MATERIALS": Materials database

- Folder "MODELS": Models database

The files have to be install in a folder directly on "C:/" : " $\mathrm{C}: /[$ FOLDER NAME]/

Caution: The folder name length must be less than ten letters and without space

START FIBERSIM 2009/LIMS Conversion Tool:

The FIBERSIM 2009/LIMS Conversion Tool is a Excel file with macro. The macros have to be activate in EXCEL.

$\checkmark$ OPEN THE FILE "FibersimLims.xlsx" to start the tool.

$\checkmark$ SELECT the sheet "MODELCONVERTER" 


\section{CONTENTS}

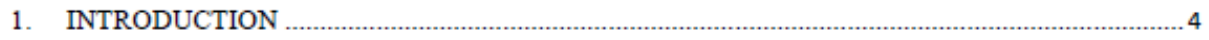

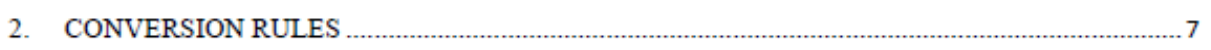

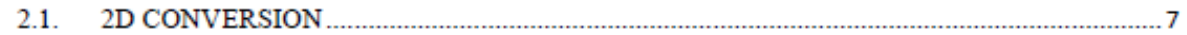

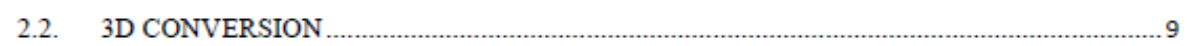

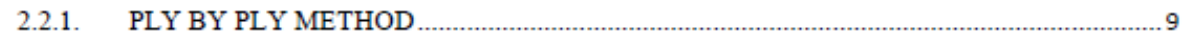

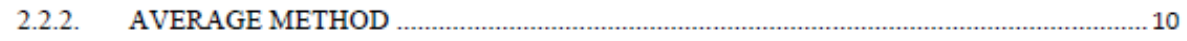

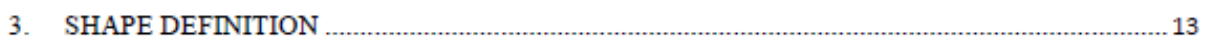

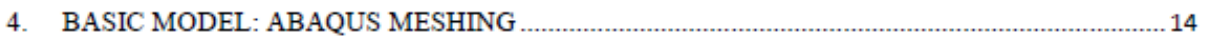

5. FIBERSIM MODEL: COMPOSITE DEFINITION

6. MATERIAL FILE: CREATION

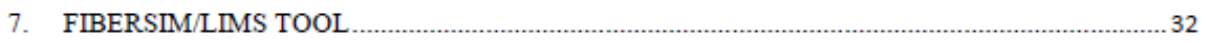

7.1. INTERFACE

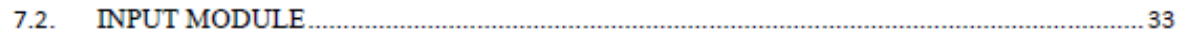

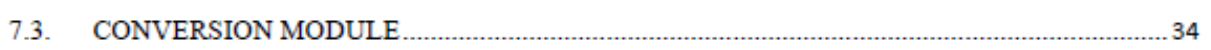

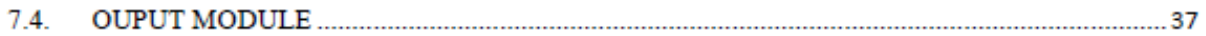

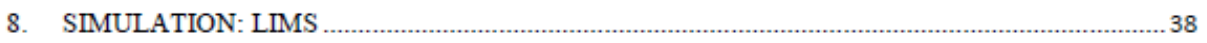

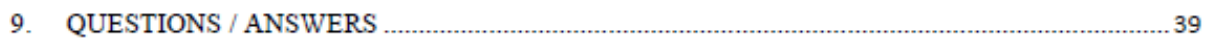

10. NOTES 


\section{(ivTHODUCTION}

\section{INTRODUCTION}

FIBERSIM/LIMS Tool use the fiber orientations from FiberSim 2009 to define the permeability and the fiber volume fraction of each LIMS element in the local element coordinate system. This conversion can be done with 2D element (in-plane permeability) or with $3 \mathrm{D}$ elements (3D permeability). The both simulations have the same inputs: material parameters, Fibersim model and Basic model (2D elements).
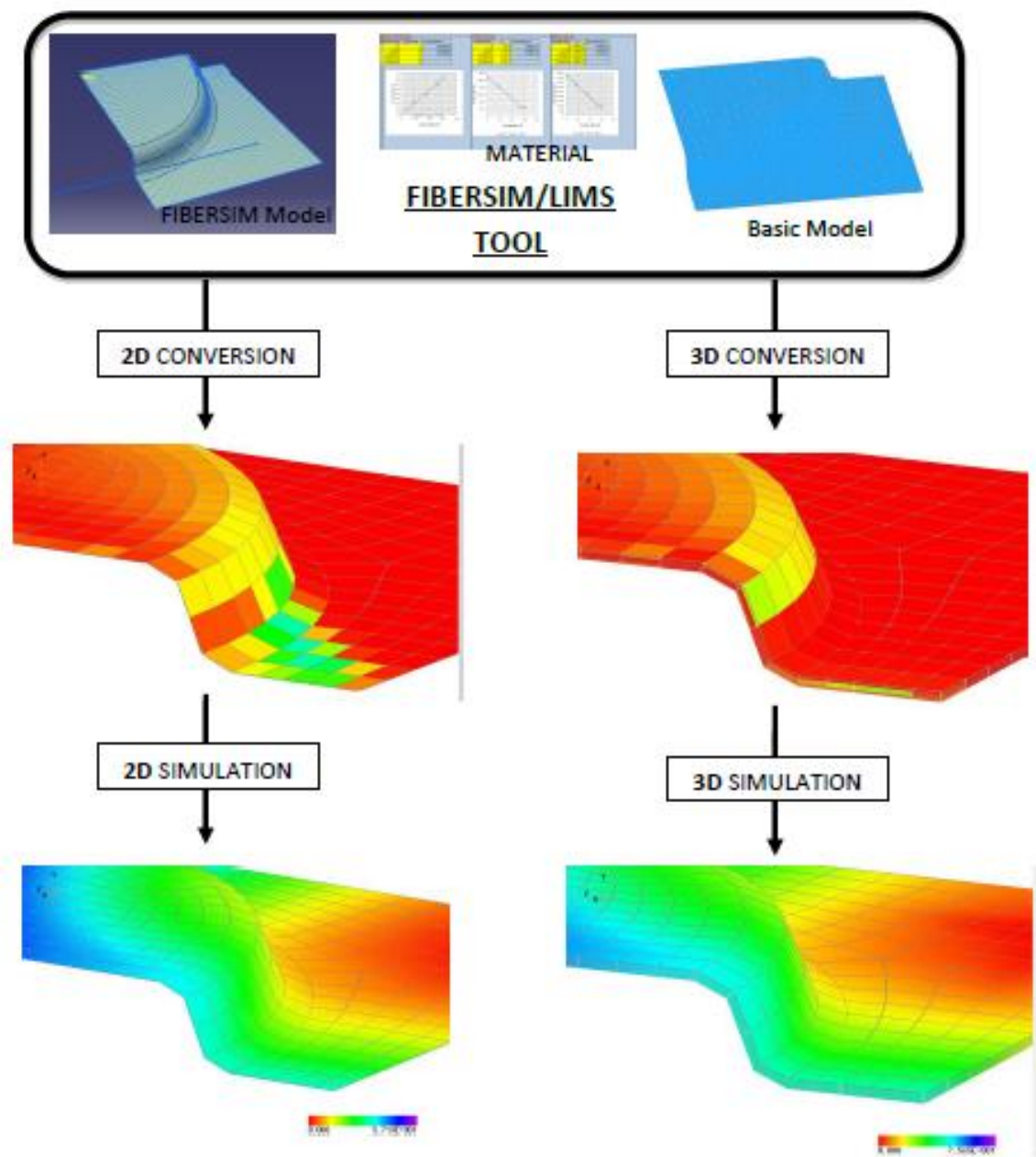


\section{INTRODUCTION}

This manual describes:

- The conversion rules for the $2 \mathrm{D}$ and $3 \mathrm{D}$ analysis.

- How to create the inputs: the material, the FiberSim model and the Basic model.

- How to use the LIMS/FIBERSIM Tool.

- How to launch a simulation with LIMS.

The general process has shown in the following figure and the general procedure next page:

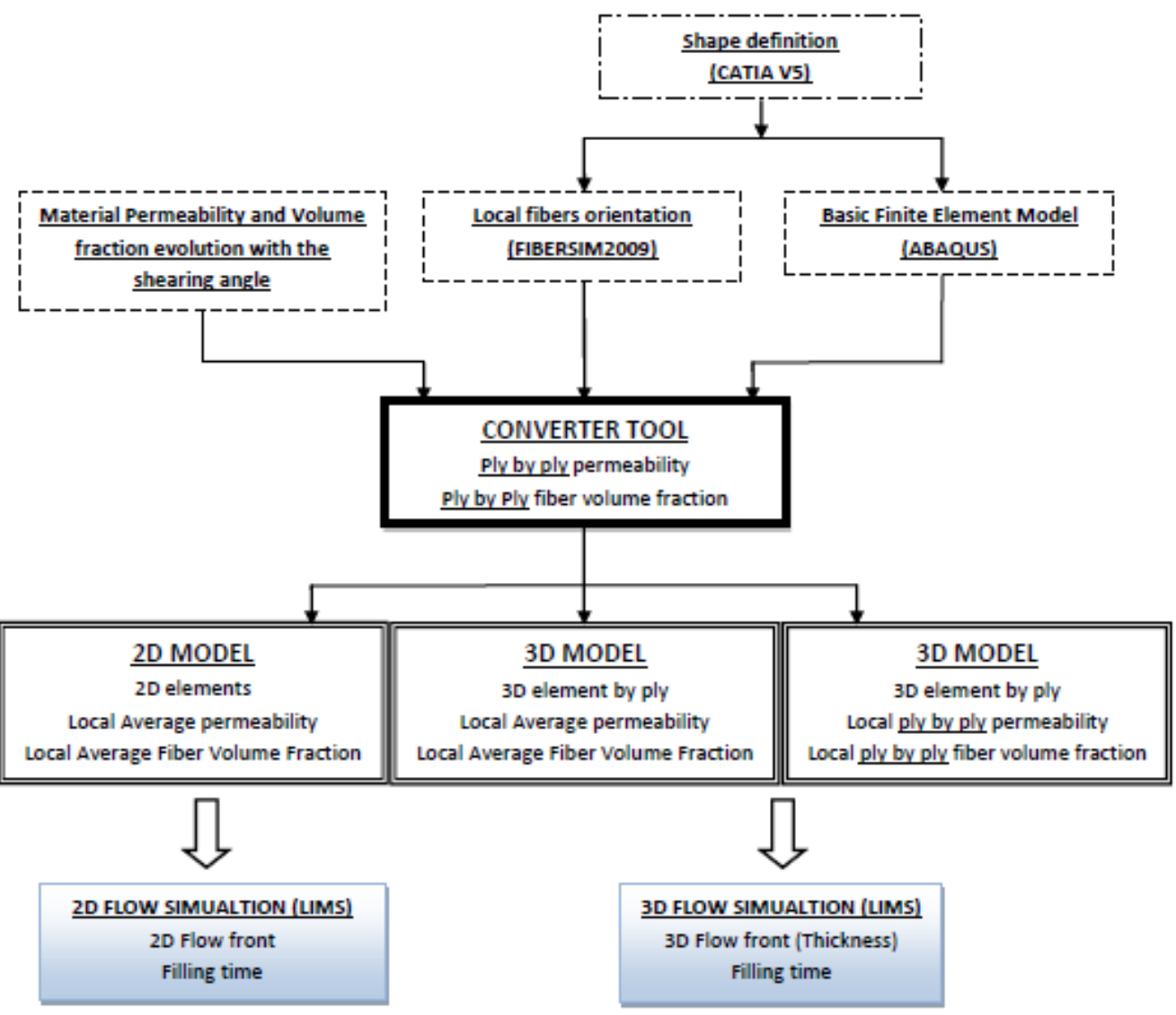

Ply by Ply flow simulation process 


\title{
GENERAL PROCEDURE
}

\author{
INPUTS: \\ 1: Create the shape with Catia V5. (Chapter 3) \\ 2: Create the Basic Model with ABAQUS. (Chapter 4) \\ 3: Create the FiberSim Model. (Chapter 5) \\ 4: Create the Bulk Material File. (Chapter 6.1) \\ 5: Create the Fiber Tow Material File. (Chapter 6.2) \\ CONVERSION PREPARATION: \\ 5: Load the material files. (Chapter 7.2) \\ 6: Load the basic model. \\ 7: Load the Fibersim Model. \\ 8: Click on "Start Conversion". (Chapter 7.3) \\ 9: Modify the number of ply if necessary. \\ 10: Choose the FiberSim model unit. \\ 11: Modify the ply thickness if necessary. \\ 12: Choose the material options.
}

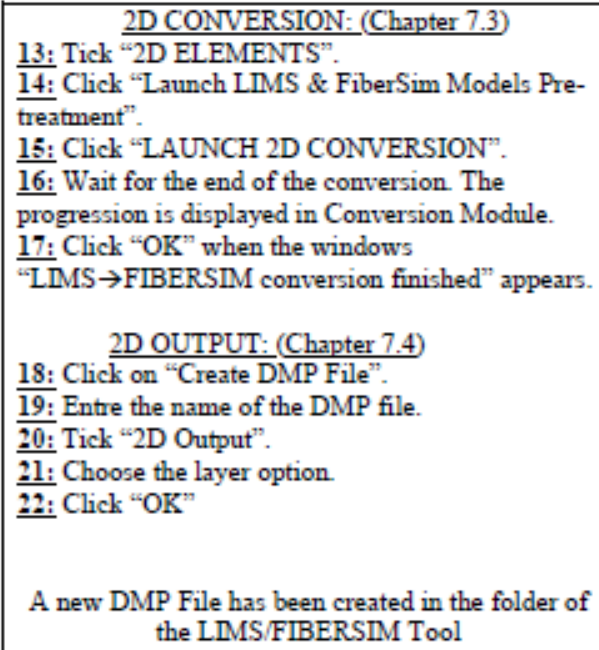

3D CONVERSION: (Chapter 7.3)
13: Tick "3D ELEMENTS".
14: Choose the "3D simulation options".
15: Click "MODEL 3D".
16: Wait the end of the model 3D creation. The
progression is displayed in the Conversion Module.
17: Click "LAUNCH 3D CONVERSION"
18: Wait the end of the conversion. The
progression is displayed in the Conversion Module.
19: Click "OK" when the windows
"LIMS $\rightarrow$ FIBERSIM conversion finished" appears.
3D OUTPUT: (Chapter 7.4)
20: Click on "Create DMP File"
21: Entre the name of the DMP file.
$\underline{22:}$ Tick "3D Output".
23: Click "OK"
A new DMP File has been created in the folder of
the LIMS/FIBERSM Tool.

LIMS SIMULATION: (Chapter 8)

24: Open the DMP file with LMS.

25: Choose "International Units"

26: Choose the injection points.

27: Define the resin viscosity

28: Launch the Simulation. 


\section{D CONVERTION}

\section{CONVERSION RULES}

\subsection{D CONVERSION}

Model: 2D elements. The elements are the basic model elements (input). For each element the permeability and the fiber volume fraction are calculated in accordance with the orientation of the all plies.

Permeability: In-plane permeability

With the $2 \mathrm{D}$ conversion only the in-plane permeability is used: $\mathrm{Kxx}, \mathrm{Kyy}$ and $\mathrm{Kxy}$. The K1, K2 and Principal Angle values are calculated with the material inputs.

For each LIMS element an average permeability is calculated with the value of each ply:

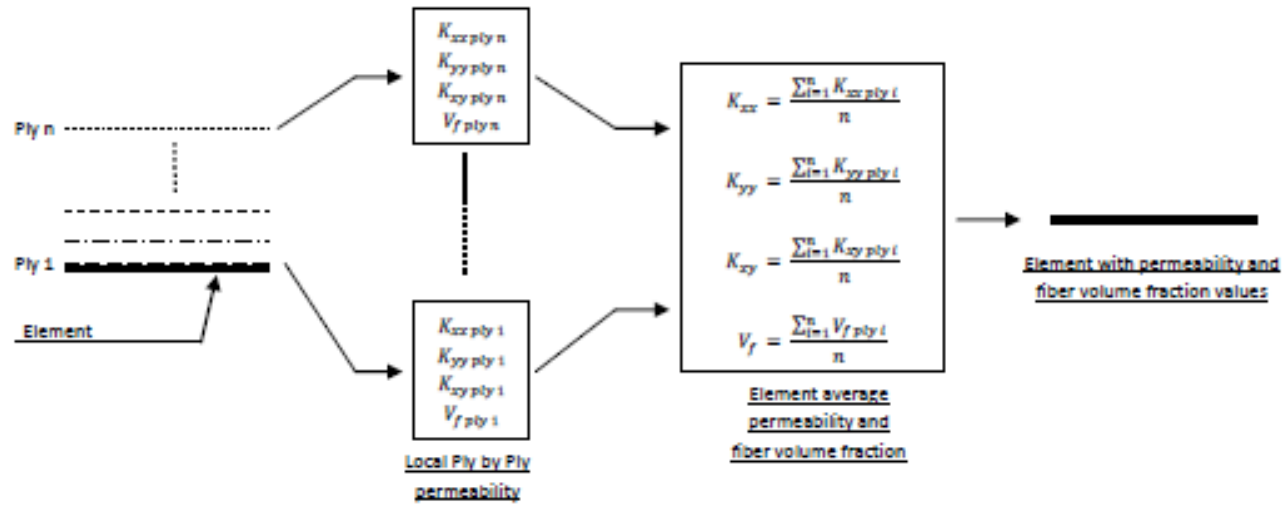

Fiber Volume Fraction:

For each LIMS element an average fiber volume fraction is calculated with the values of each ply:

$$
\begin{gathered}
V f_{\propto}=\frac{V f_{\text {initial }}}{\cos \propto} \text { with } \propto: \text { shearing angle } \\
V_{f}=\frac{\sum_{i=1}^{n} V_{f} \text { ply } i}{n}
\end{gathered}
$$


2.1.1. Modeling Dual Scale Flow and Tow Saturation

One dimensional tow elements may be created and appended to each node in the $2 \mathrm{D}$ mesh given the properties defined by the appropriate Fiber Tow Material file.
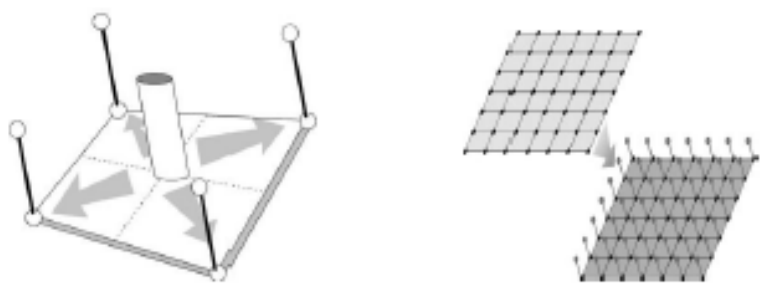

Each tow element is assigned a volume determined by the total volume of surrounding elements summed through the thickness of the material.

The tow element permeability is assigned according to the average shear angle of the surrounding $2 \mathrm{D}$ elements and the tow permeability model described in the Fiber Tow Material file in the form of:

$$
K_{\text {tow }}=\theta_{0}(1-\alpha)^{\theta_{1}}
$$

with $\alpha$ the local element shear angle in radians. 


\section{D CONVERTION}

\subsubsection{CONVERSION}

Model: 3D elements. For each basic model elements FIBER/LIMS Tool create one or more 3D element by ply. The permeability and the fiber volume fraction are calculated with the corresponding ply for each $3 \mathrm{D}$ element.

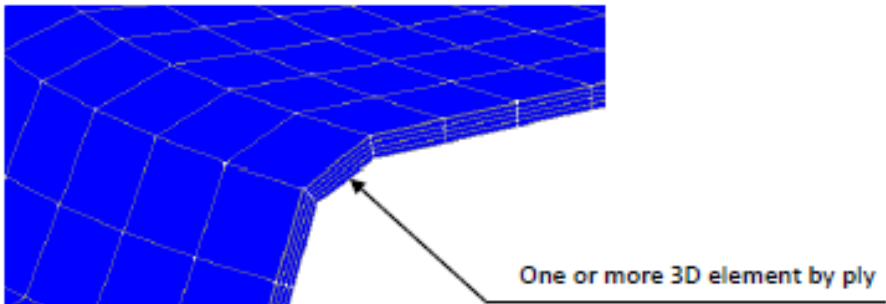

\subsubsection{PLY BY PLY METHOD}

With this method the permeability and the fiber volume fraction are calculated with the corresponding ply for each $3 \mathrm{D}$ element.

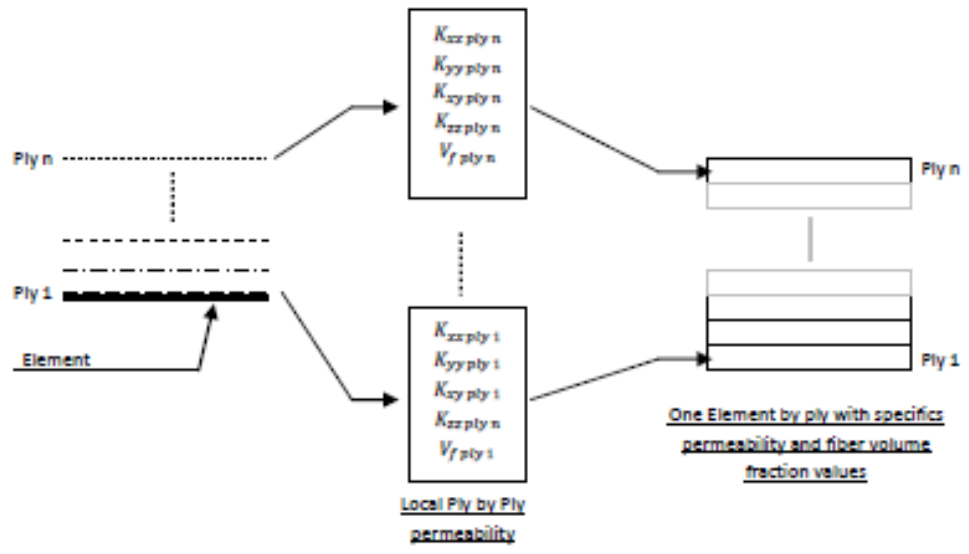

Permeability: $3 \mathrm{D}$ permeability.

- Kxx, Kyy and $\mathrm{Kxy}$ are calculated for each element with the material input and the conresponding layer. 


\section{D CONVERTION}

- $\mathrm{K} z z$ is calculated with the following formula for each element:

$$
\begin{gathered}
K_{z z} \text { initial }=\frac{K_{1} \text { initial }}{100} \\
K_{z z}=A \frac{\left(1-V f_{\infty}\right)^{3}}{V f_{\circ}^{2}} \text { with } A=K_{z z} \text { initial } \frac{V f_{\text {initial }}{ }^{2}}{\left(1-V f_{\text {tnitial }}\right)^{3}}
\end{gathered}
$$

- $\mathrm{K} z \mathrm{y}=\mathrm{Kzx}=0$

Fiber Volume Fraction: The fiber volume fraction is calculated for each element with the corresponding shearing angle :

$$
V f_{\propto}=\frac{V f_{\text {initial }}}{\cos \alpha} \text { with } \propto: \text { shearing angle }
$$

\subsubsection{AVERAGE METHOD}

With this method a local average, on the thickness, of the permeability and the fiber volume fraction is calculated and applied on all the elements.

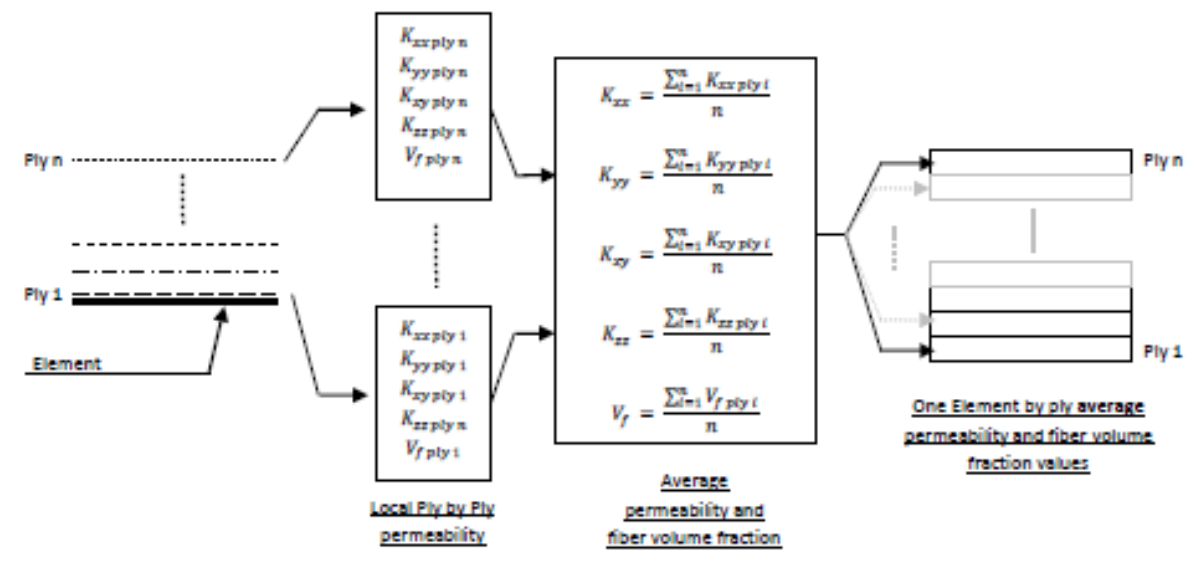

Permeability: $3 \mathrm{D}$ permeability.

- $\mathrm{Kxx}, \mathrm{Kyy}$ and $\mathrm{Kxy}$ are calculated for each ply with the material inputs and an average is done to be applied on all the elements on the thickness. 


\section{D CONVERSION}

- $\mathrm{K} z z$ is calculated with the following formula for each ply. An average is done and applied on all the elements on the thickness:

$$
\begin{gathered}
K_{z z} \text { initial }=\frac{K_{1} \text { initial }}{100} \\
K_{z z \text { ply } i=A \frac{\left(1-V f_{\alpha}\right)^{3}}{V f_{\alpha}{ }^{2}}} \text { with } A=K_{z z} \text { initial } \frac{V f_{\text {initial }}{ }^{2}}{\left(1-V f_{\text {initial }}\right)^{3}} \\
K_{z z}=\frac{\sum_{i=1}^{n} K_{z z \text { ply } i}}{n}
\end{gathered}
$$

- $\mathrm{K} z \mathrm{y}=\mathrm{Kzx}=0$

\section{$>$ Fiber Volume Fraction:}

For each LIMS element an average fiber volume fraction is calculated with the values of each ply:

$$
\begin{gathered}
V f_{\propto}=\frac{V f_{\text {initial }}}{\cos \alpha} \text { with } \propto \text { : shearing angle } \\
V_{f}=\frac{\sum_{i=1}^{n} V_{f \text { ply } i}}{n}
\end{gathered}
$$

2.2.3. Modeling Dual Scale Flow and Tow Saturation

One dimensional tow elements may be created and appended to each node in the $3 \mathrm{D}$ mesh given the properties defined by the appropriate Fiber Tow Material file.
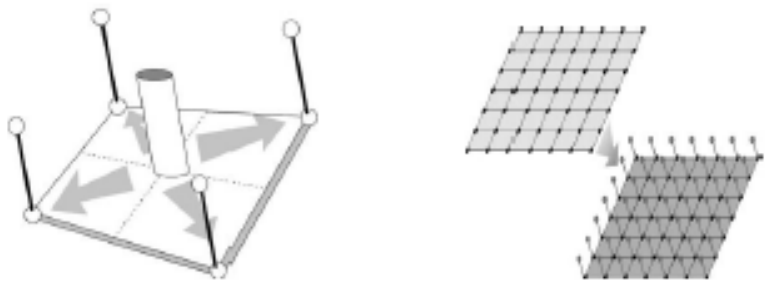

Each tow element is assigned a volume determined by the volume of surrounding elements in the $3 \mathrm{D}$ mesh. 
The tow element permeability is assigned according to the average shear angle of the surrounding $3 \mathrm{D}$ elements and the tow permeability model described in the Fiber Tow Material file in the form of:

$$
K_{\text {tow }}=\theta_{0}(1-\alpha)^{\theta_{1}}
$$

with $\alpha$ the local element shear angle in radians. 


\section{SHAPE DEFINIION}

3. SHAPE DEFINITION

\section{CAUTION}

- The shape has to be a surface.

The definition of the shape is done with CATIA V5. Please refer to the CATIA manual to design the surface.

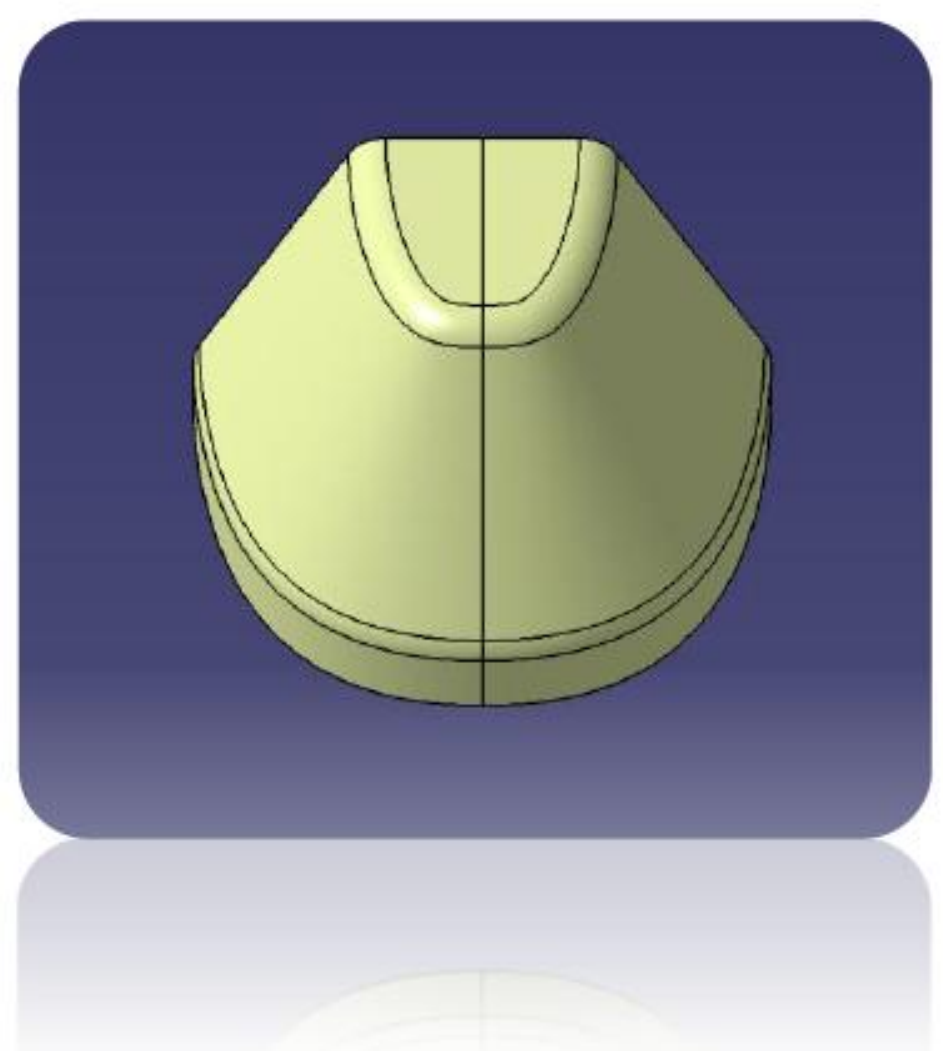


4. BASIC MODEL: ABAQUS MESHING

\section{$\triangle$ CAUTION}

- Only linear SHELL element can be used.

- For 2D and 3D study the same mesh is defined (For the 3D study, the 3D elements will be created by FIB/LIMS tool).

- For 3D study, the elements dimensions has to be in accordance with the ply thickness (h) : $\underline{\mathbf{5}} \mathbf{x} \mathbf{h}<$ elements dimensions $<\mathbf{2 0} \times \mathbf{h}$

- A convergence study has to be done to confirm the elements dimensions.

A mesh of the part has to be done to simulate the injection. This mesh is an input of the FIB/LIMS conversion tool.

\section{PROCEDURE:}

EXPORT FILE FROM CATIA V5 (.IGS):

$\checkmark$ / File / Save As ... :

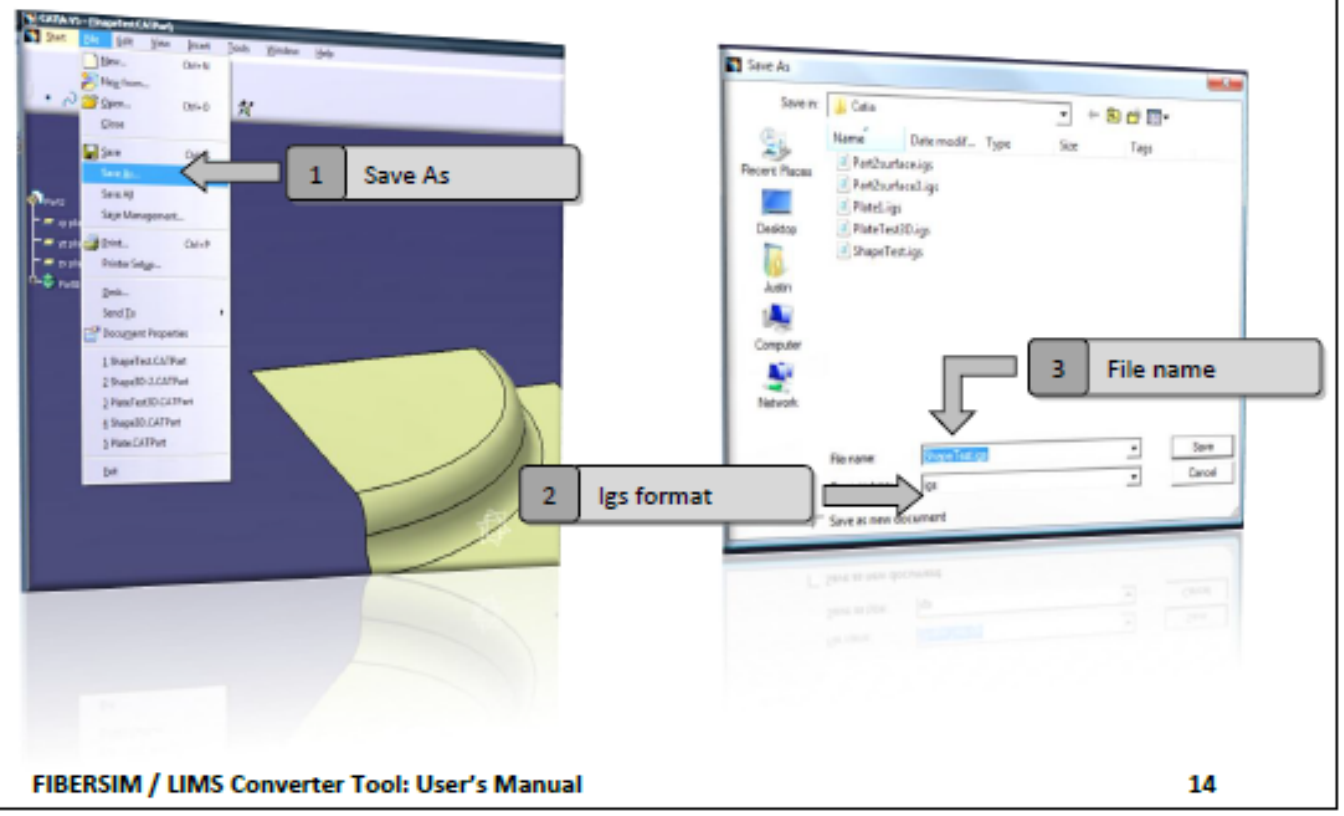




\section{BASIC FINITE ELEMENT MODEL: ABAQUS MESHING}

\section{MMPORT IGS FILE IN ABAQUS :}

$\checkmark$ Open ABAQUS

$\checkmark$ Create a new part

$\checkmark /$ File/Import / Part...:
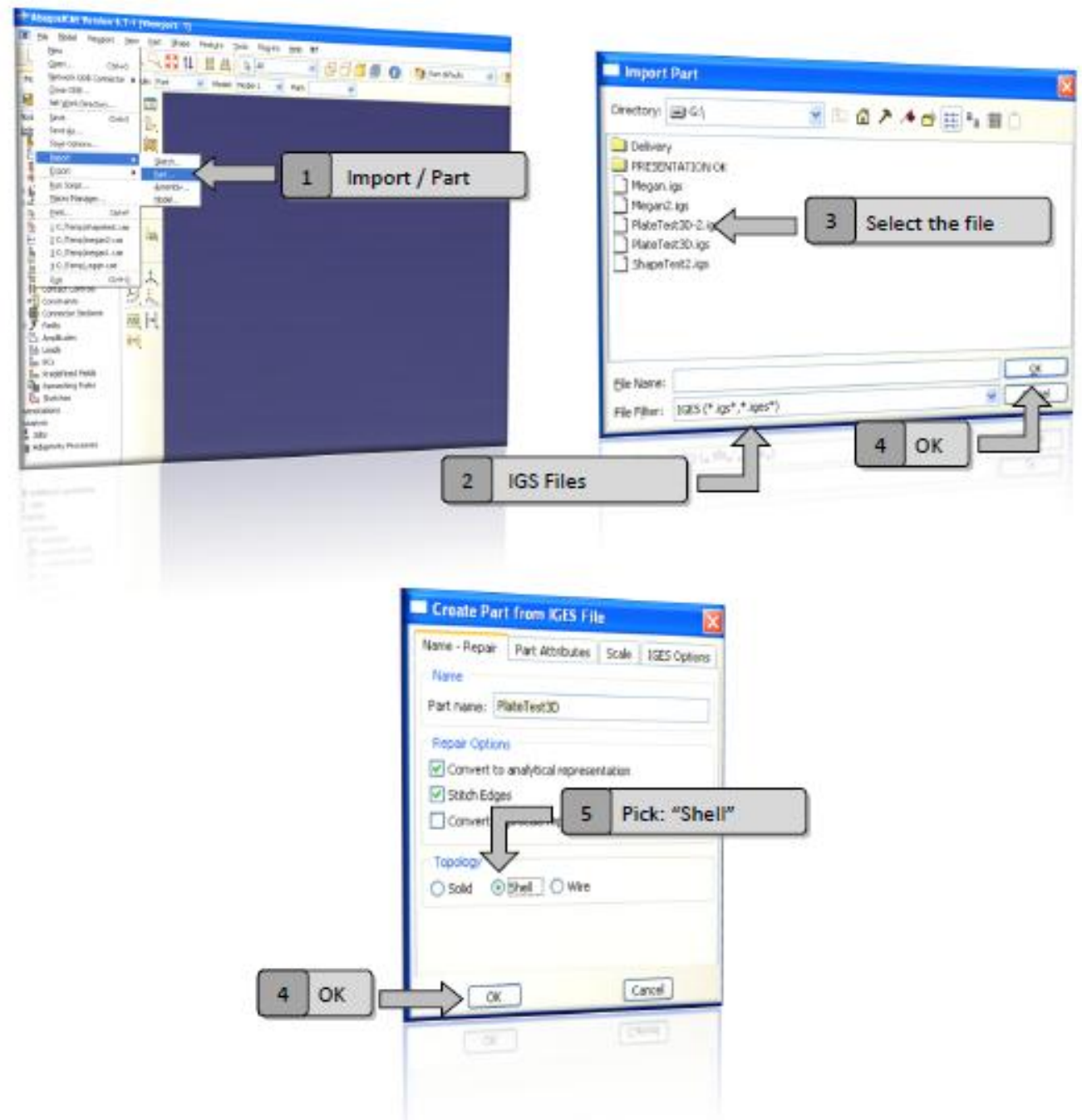


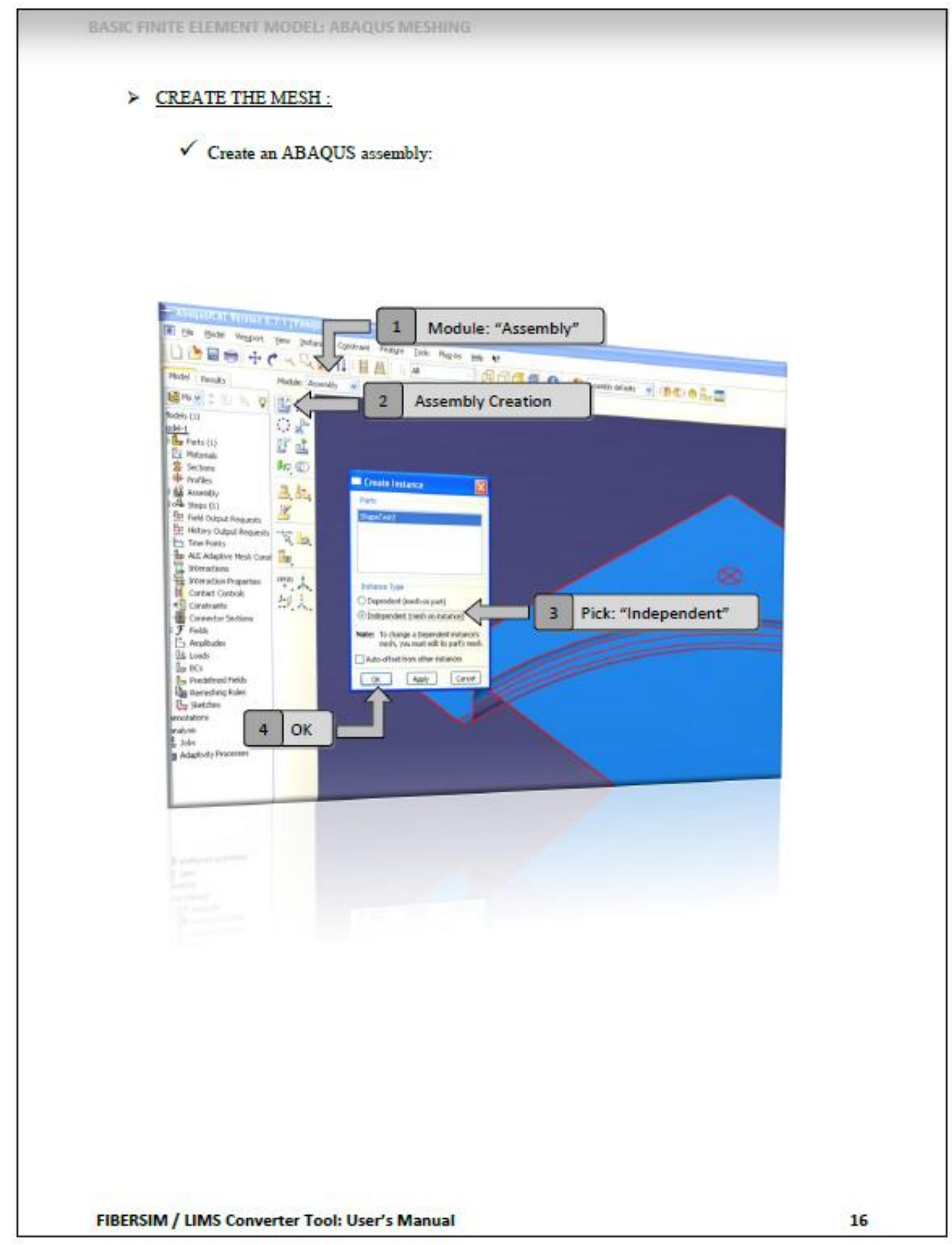


Define the size of the elements:

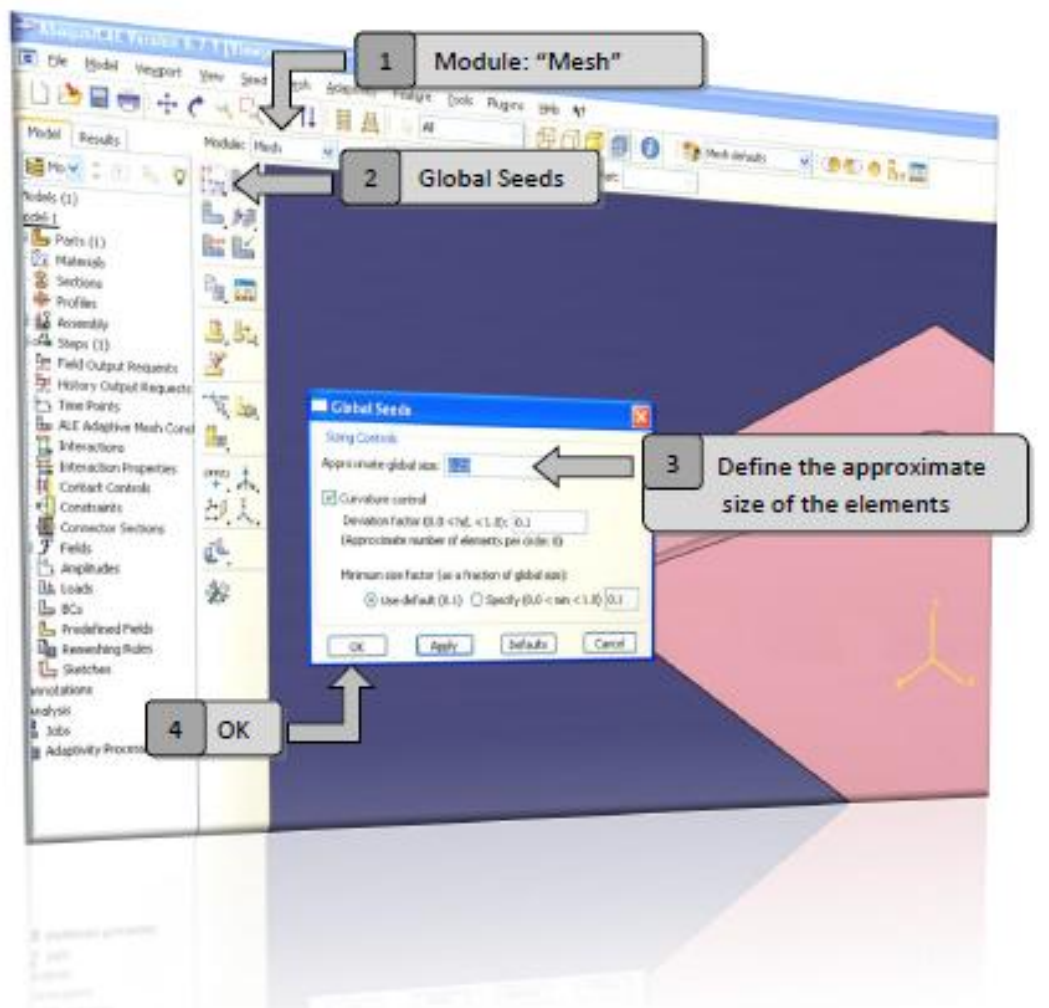

Other options to define the elements size:

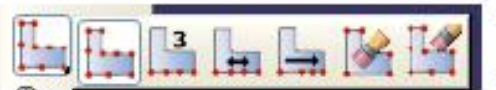

Stay click on the icon to obtain more options

3

1... : Define locally the number of elements on one or more edges.

$\rightarrow$

: Define locally the size of the elements on one or more edges 
BASIC FINITE ELEMEENT MOOELL ABRQUS MESHING

Select the elements format:

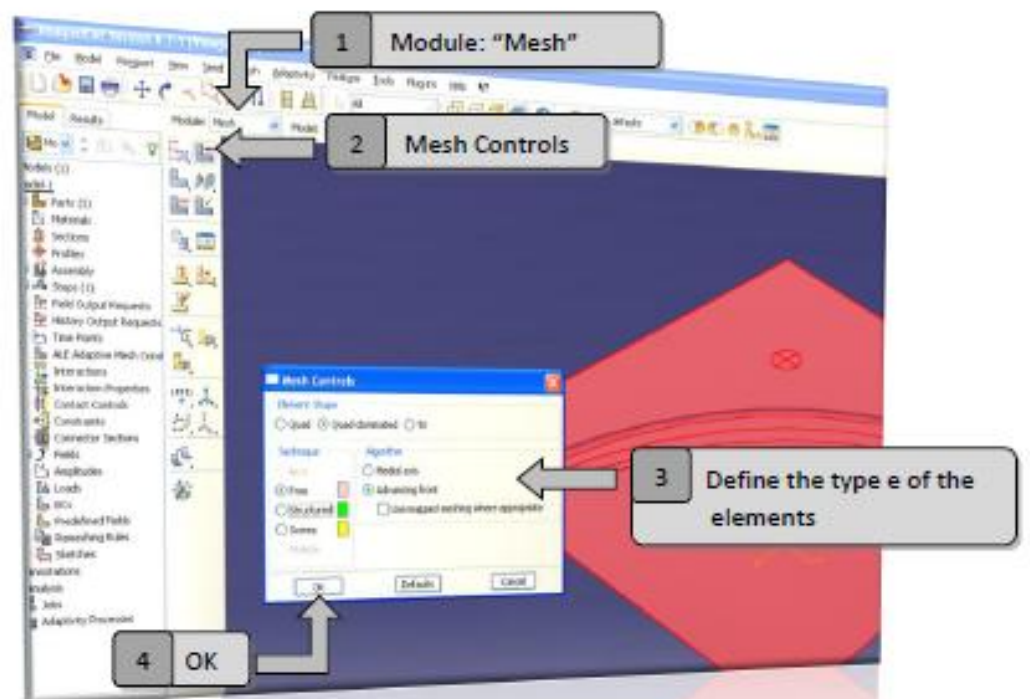


BASIC FIUTE GEMIEITT MODELI ABAQUS MIESHING

$\checkmark$ Create the mesh :
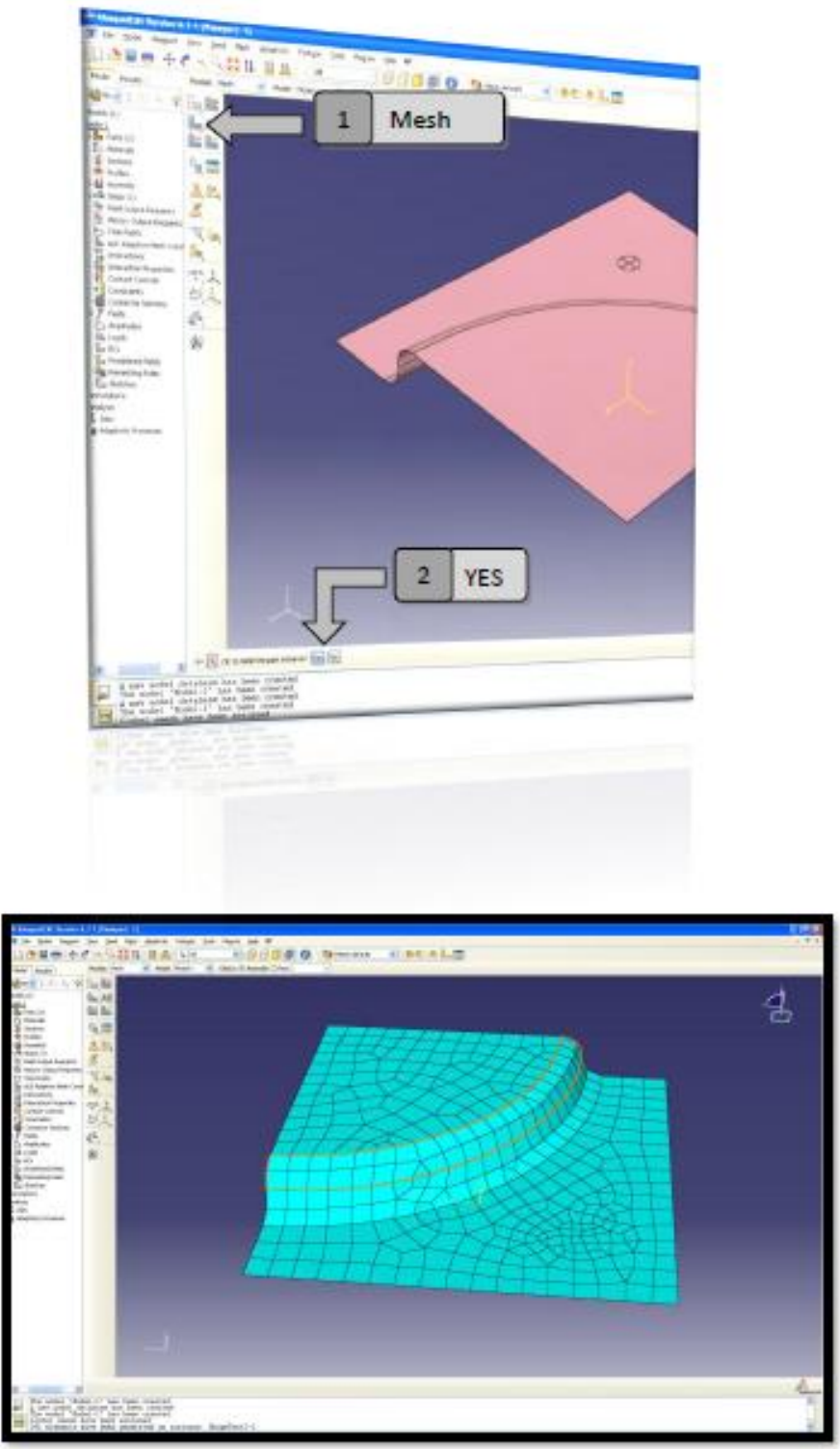

The mesh is created 


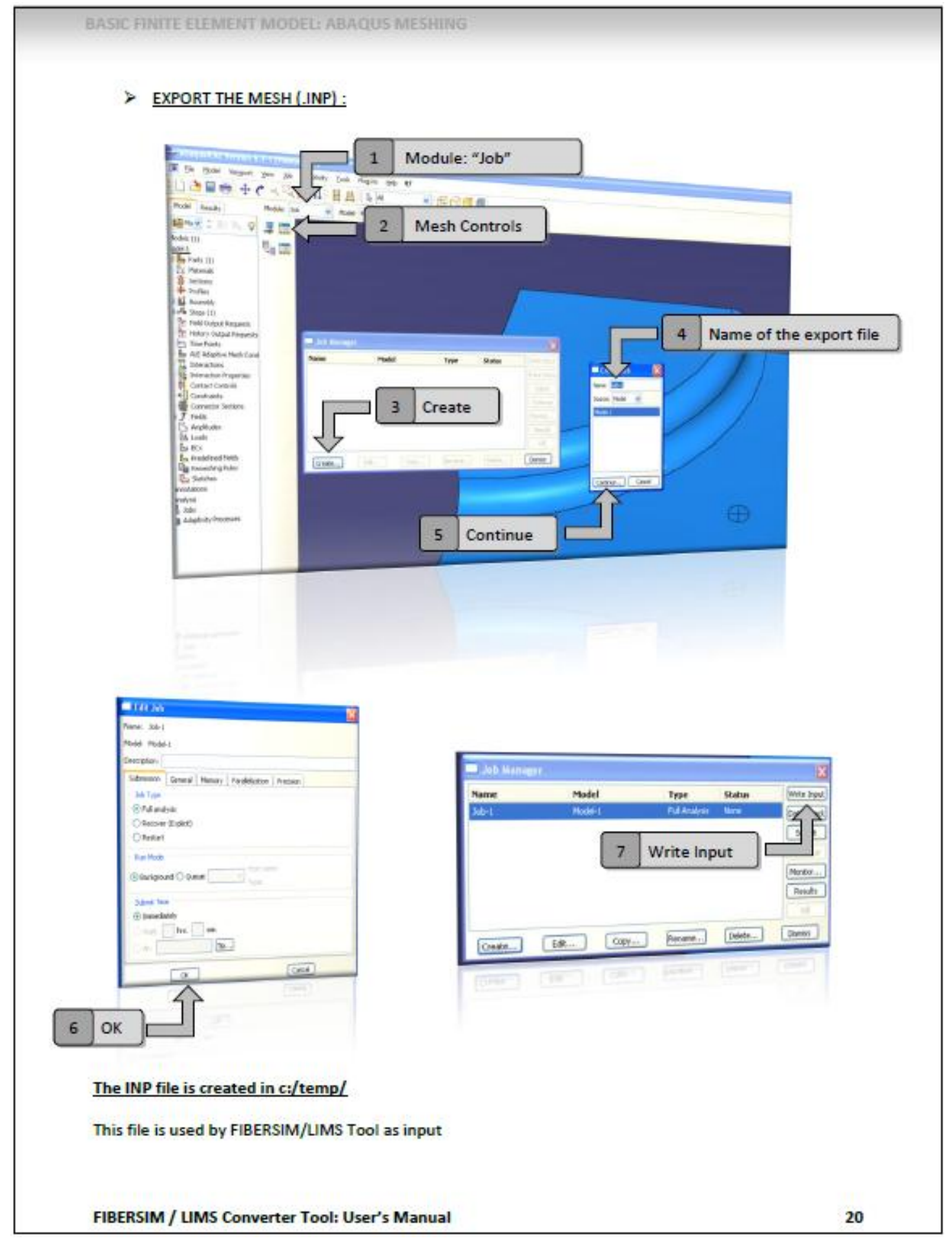




\section{FIBERSIM MODEL: COMPOSITE DEFINITION}

\section{FIBERSIM MODEL: COMPOSITE DEFINITION}

\begin{tabular}{|l|}
\hline CAUTION \\
\hline - Only constant thickness can be used with FIBER/LIMS tool. \\
- The number of FIBERSIM element has to be approximately at least \\
twice of the number of LIMS elements. ( A visual check is sufficient)
\end{tabular}

The composite definition is realized with FIBERSIM 2009 in CATIA V5. The output XML file is used by FIB/LIMS tool.

The composite definition is divided in five steps:

- Boundary lines creation

- The laminate creation

- The rosette creation

- The plies definition

- Export file (.xml) creation

PROCEDURE:

\section{BOUNDARY LINES CREATION:}

To use the FIBRSIM Mesh with FIBERSIM/LIMS Tool an extrapolation of the surface has to be done. Two boundary lines (net and extended) are created. 
FBERSIM MODEL COMPOSTIE DEFANITON

Create the net boundary line:

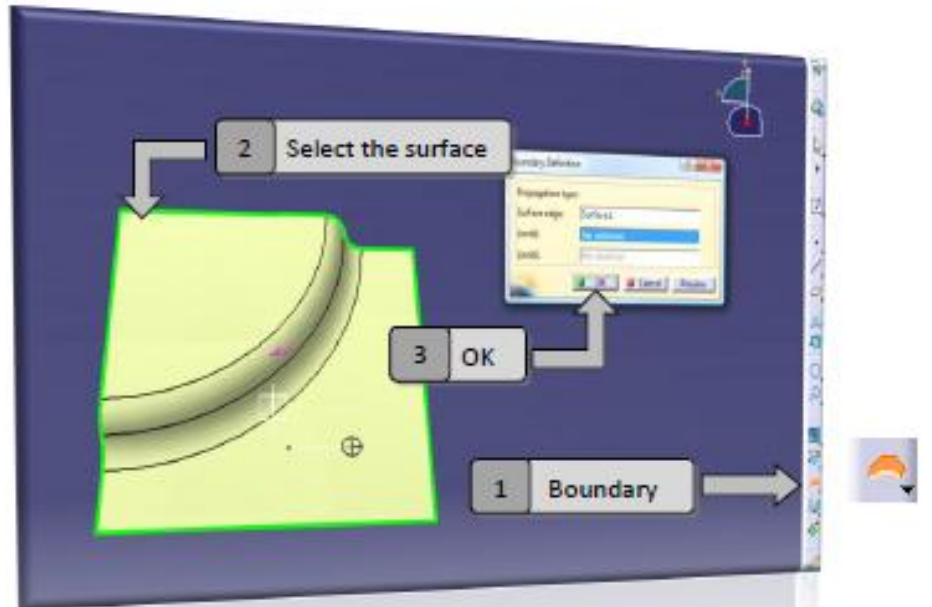

Extrapolate the surface:

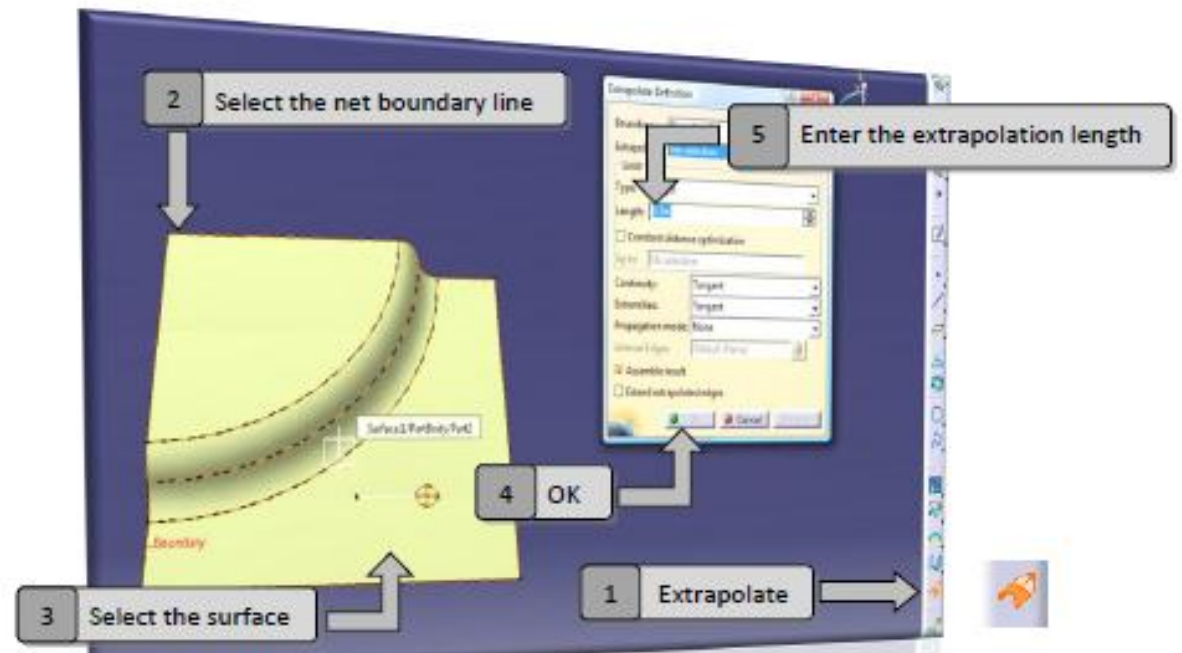




\section{ARERSIM MODEL: COMPOSTIE DEFINITION}

$\checkmark$ Create the extended boundary line:

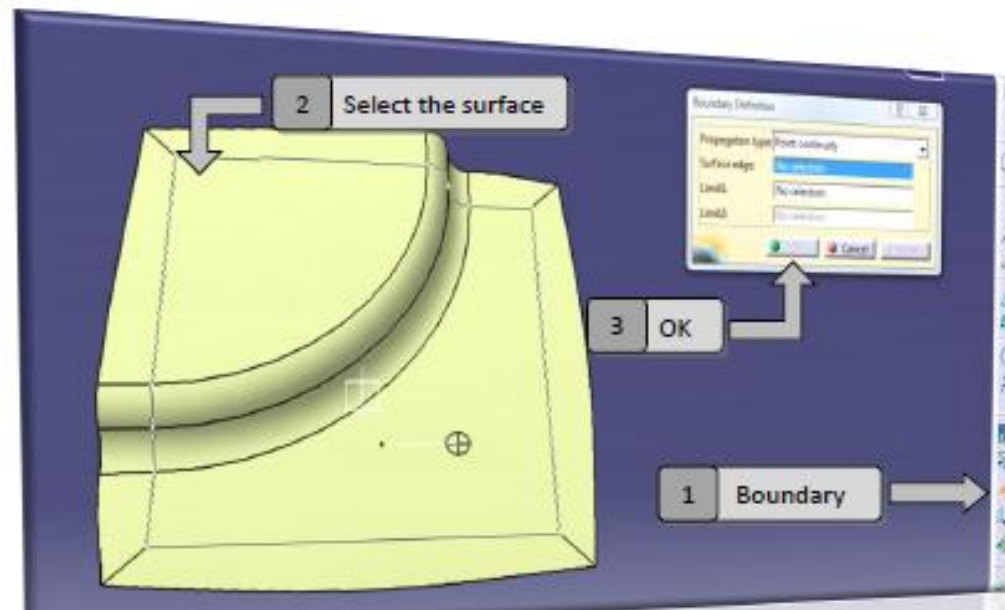

\section{LAUNCH FIBERSIM :}
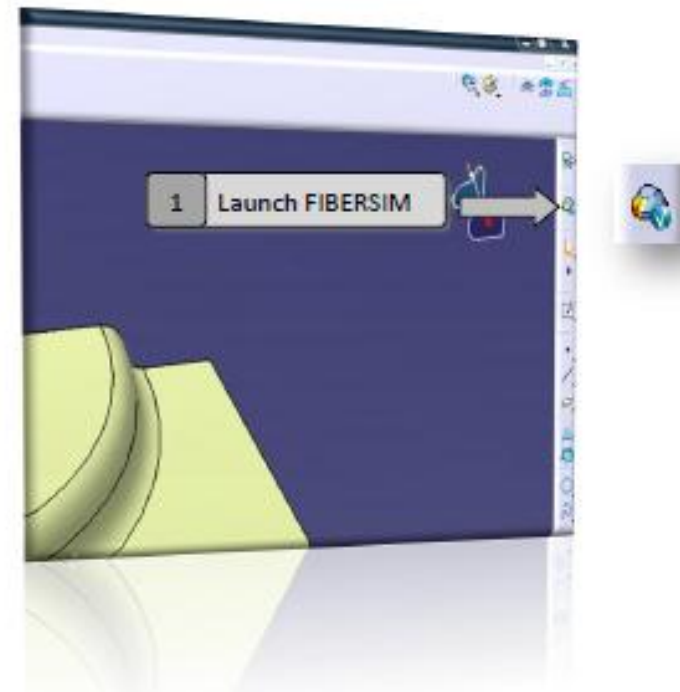


\section{FIBERSIM MODEL: COMPOSITE DEFINITION}

\section{$>$ CREATE A NEW LAMINATE:}

Create the laminate:

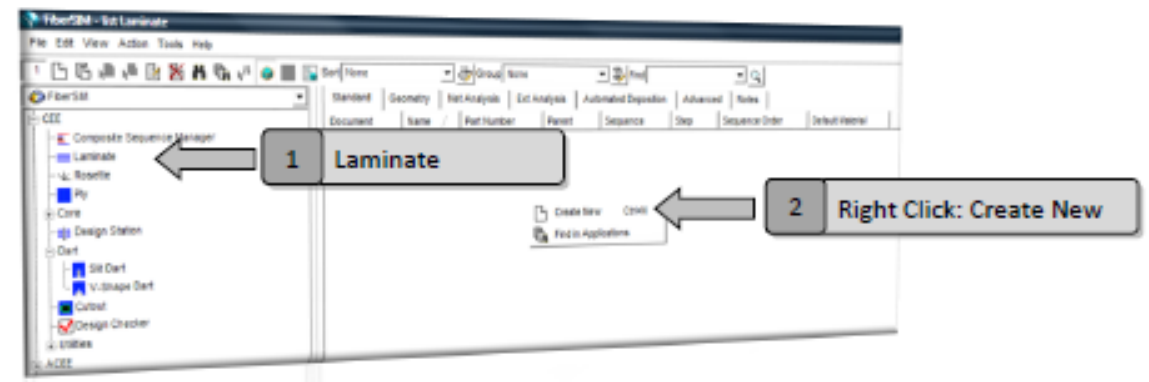

$\checkmark$ Select the material:

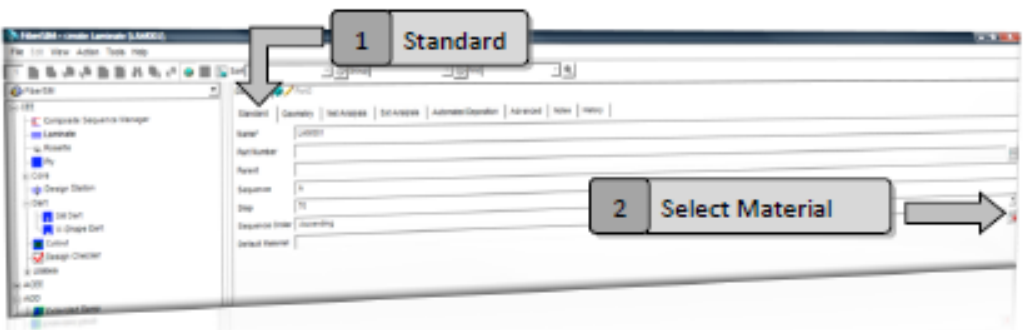

$\checkmark$ Select the surface:

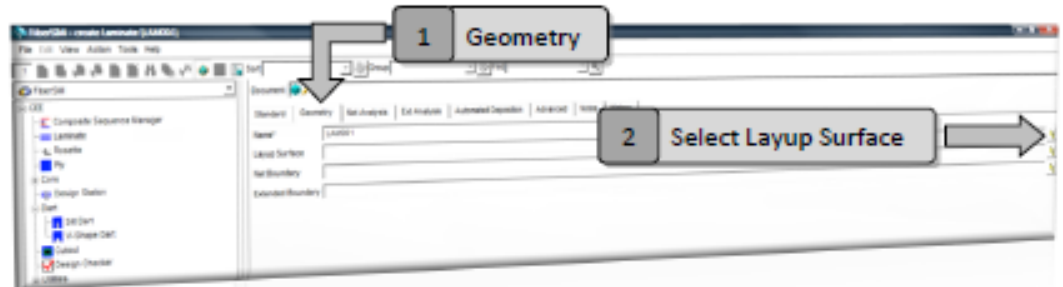




\section{FiBERSIM MIODEL: COMPOSTE DEFINITIN}


$\checkmark$ Valid the laminate: Click OK 


\section{FHBERSIM MIODEL: COMIPOSITE DEFINITON}

$>$ CREATE A ROSETTE:

$\checkmark$ Create the rosette:
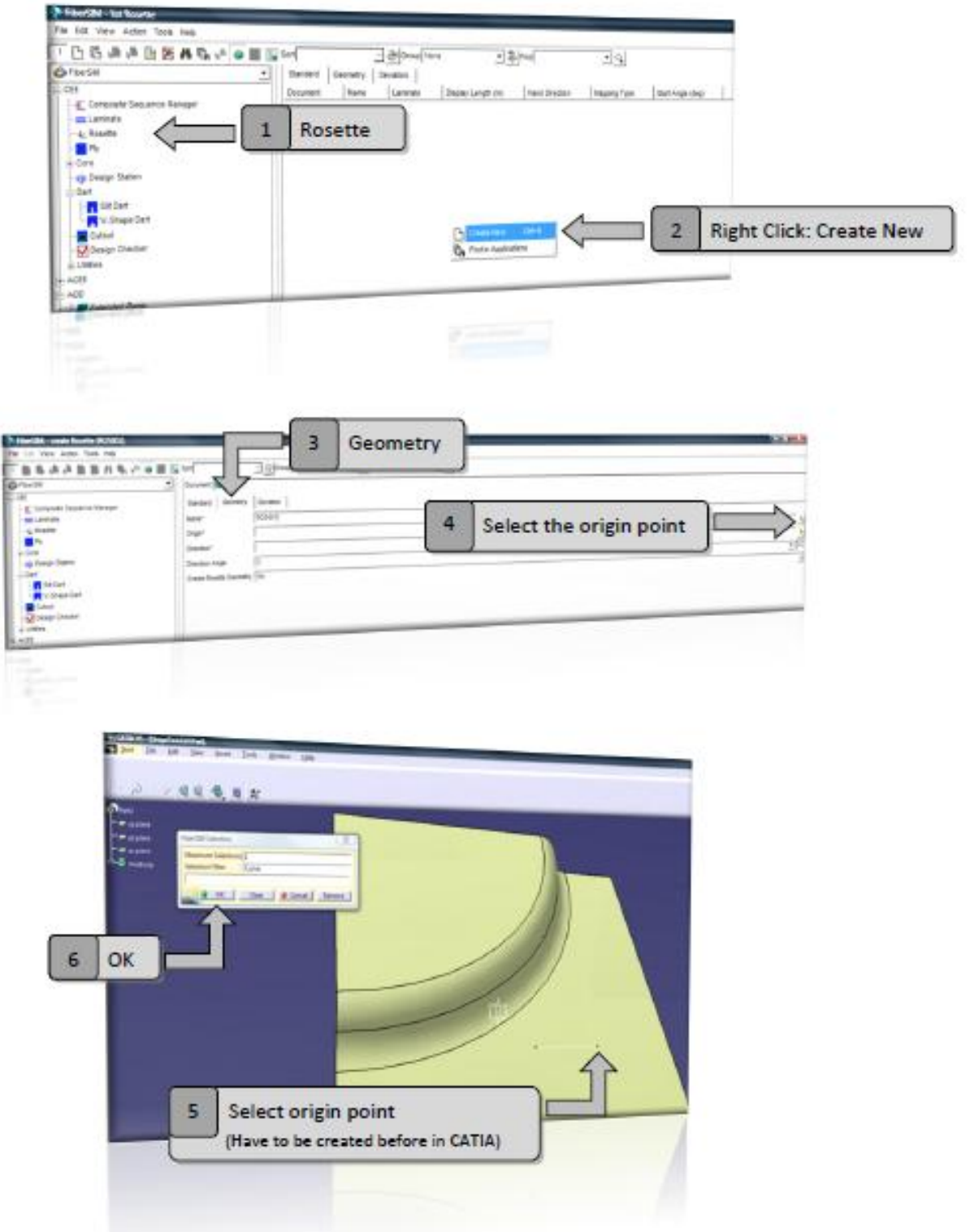

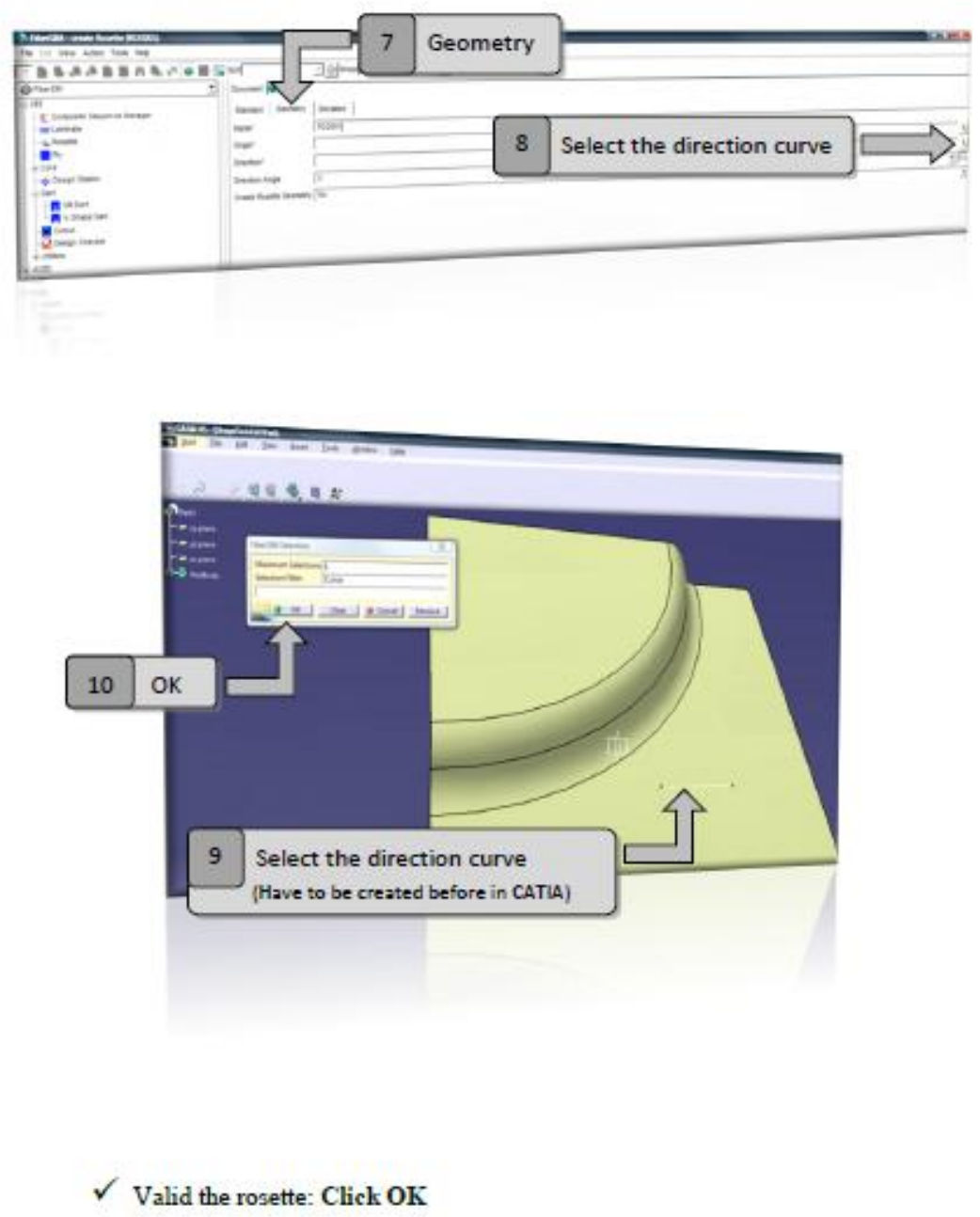


\section{FIBERSIM MODEL: COMPOSITE DEFINITION}

$>$ CREATE PLIES:

The following procedure has to be repeated for each ply.
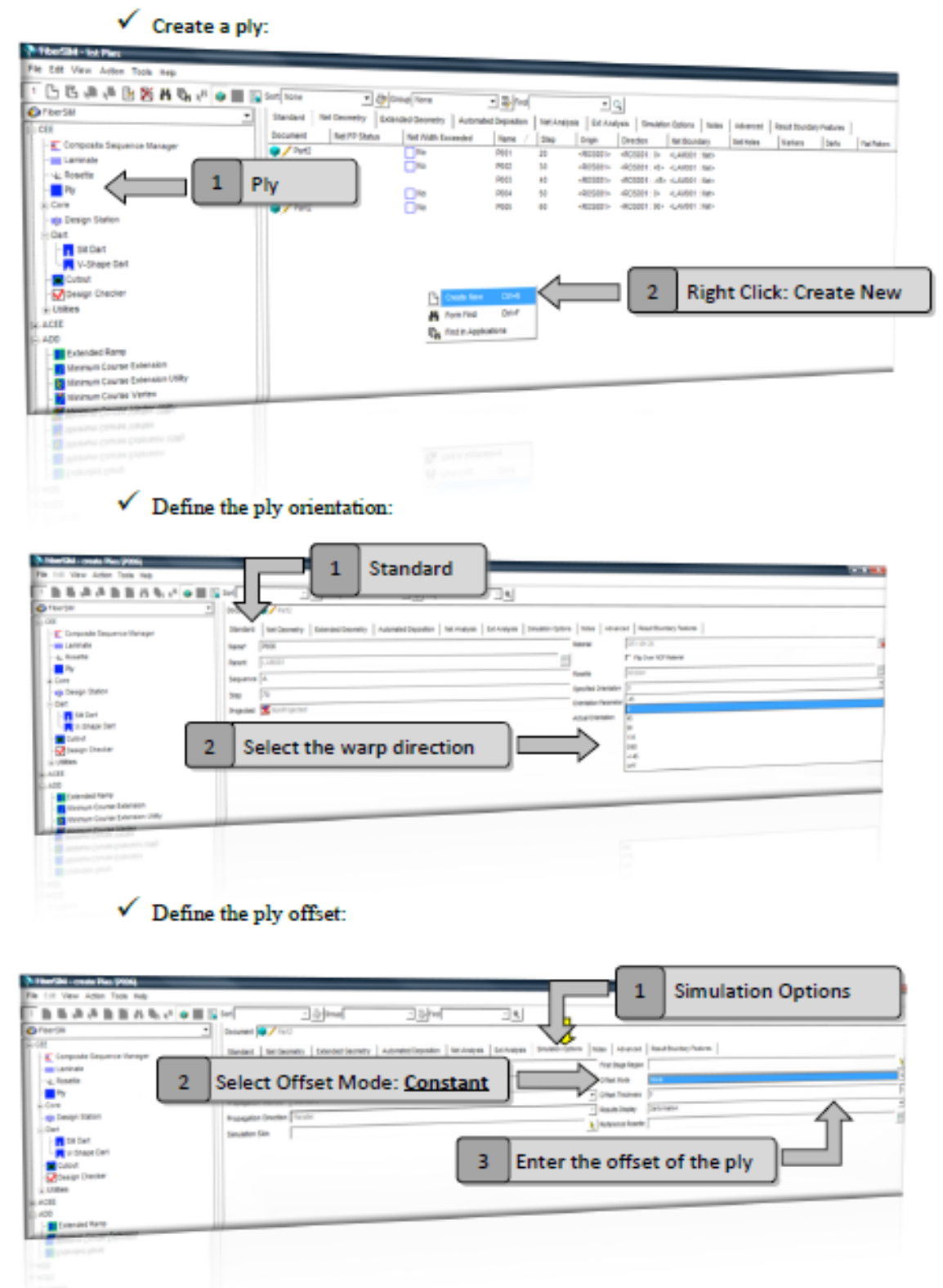


\section{ABEASIM MODEL COMPOSTE DEFINUTION}

Define the mesh thinness:
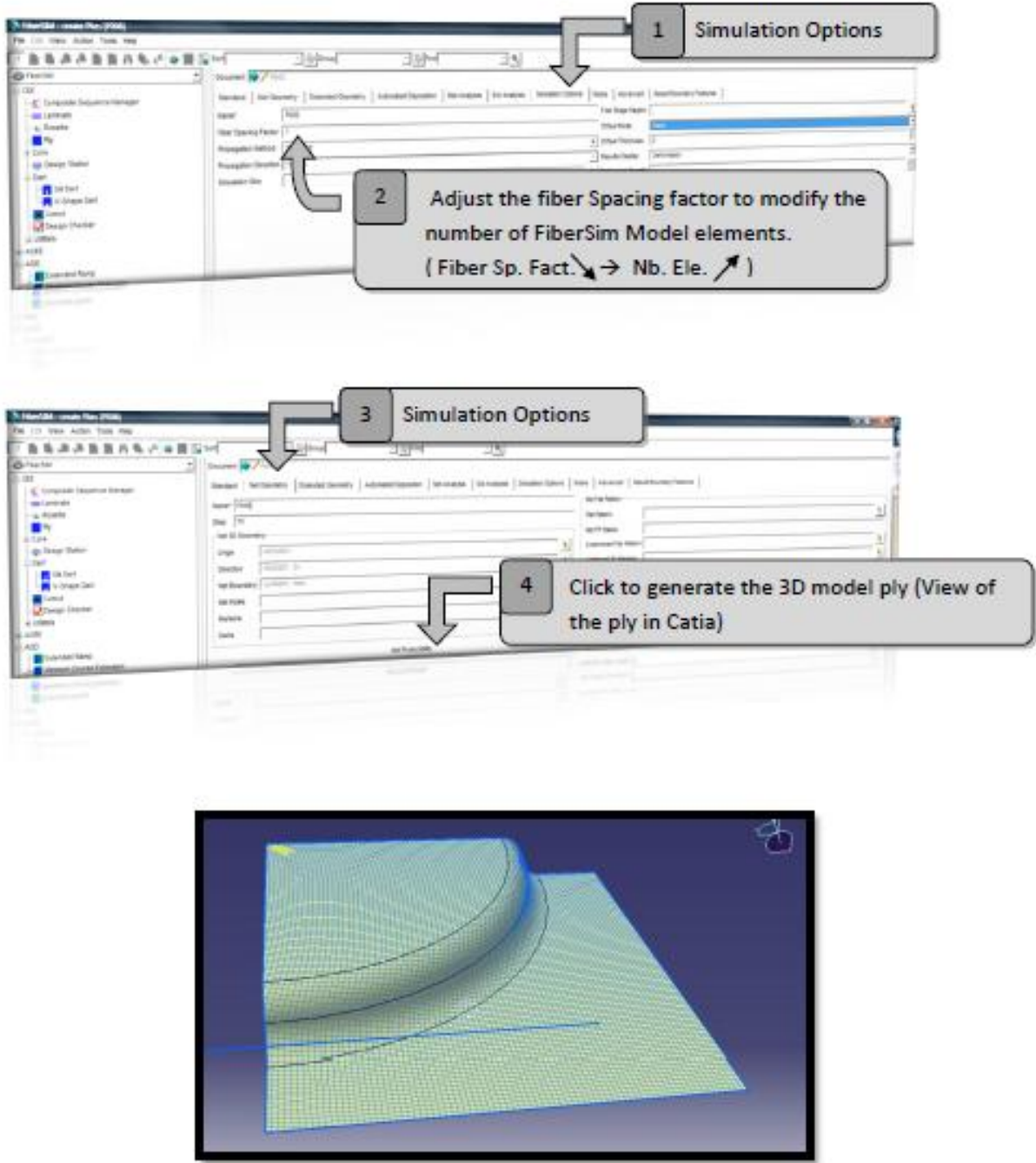

Valid ply: Click OK 


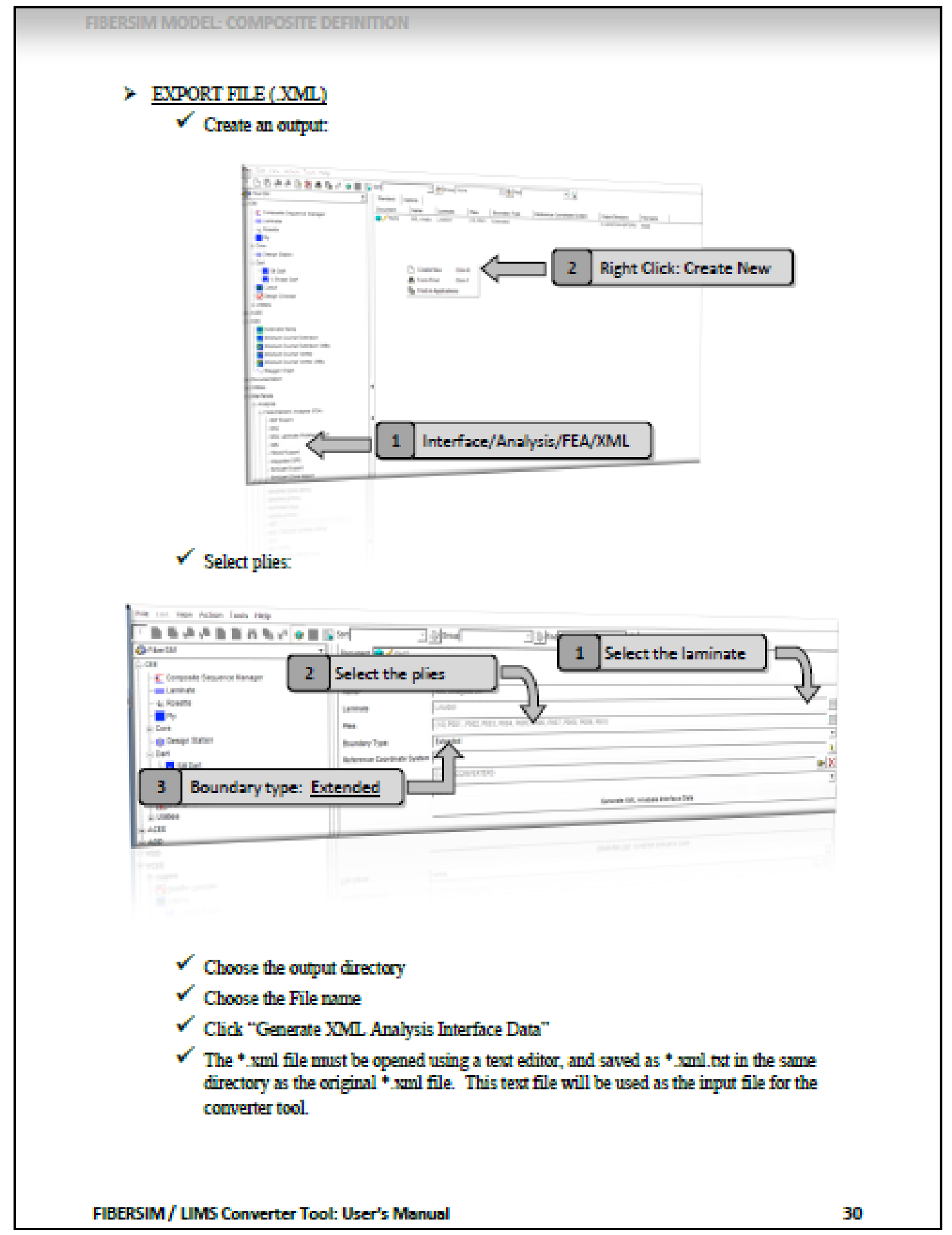




\section{MATERIAL FILE: CREATION}

\section{$\triangle$ CAUTION}

- The materials values have to be issued from experimental results.

The material files are an EXCEL file with the following information:

- Initial fiber volume fraction (between 0 and 1 )

- The thickness of $1 \mathrm{ply}$ (meter)

- The permeability value $\mathrm{K}_{1}\left(\mathrm{~m}^{2}\right)$ evolution with the shearing angle (Radian).

- The permeability value $\mathrm{K}_{2}\left(\mathrm{~m}^{2}\right)$ evolution with the shearing angle (Radian).

- The Principal Angle (Radian) evolution with the shearing angle (Radian).

- The tow permeability $\mathrm{K}_{10 \mathrm{w}}(\mathrm{m} 2)$ evolution with the shearing angle (Radian).

The volume fraction evolution is calculated by FIBERSIM/LIMS Tool (See chapter 2).

For the 3D study the values of $\mathrm{Kzz}, \mathrm{Kzy}$ and $\mathrm{Kzx}$ are calculated by FIBERSM/LIMS Tool, see chapter 2 .

$>$ How to create a material:

$\checkmark$ Open an existing file or the material template file in the folder "MATERIALS".

$\checkmark$ Change the fiber volume fraction and thickness values (Yellow Cells).

$\checkmark$ Complete the tables of K1, K2 and Principal Angle evolutions (Yellow Cells).

$\checkmark$ Change the permeability model fit parameters $\theta_{0}$ and $\theta_{1}$ for $K_{1}, K_{2}$, and $K_{100}$ to the empirically determined values

$\checkmark$ Save the file with the name of the material.

$\checkmark$ Close the file.

The following figure shows the material file:

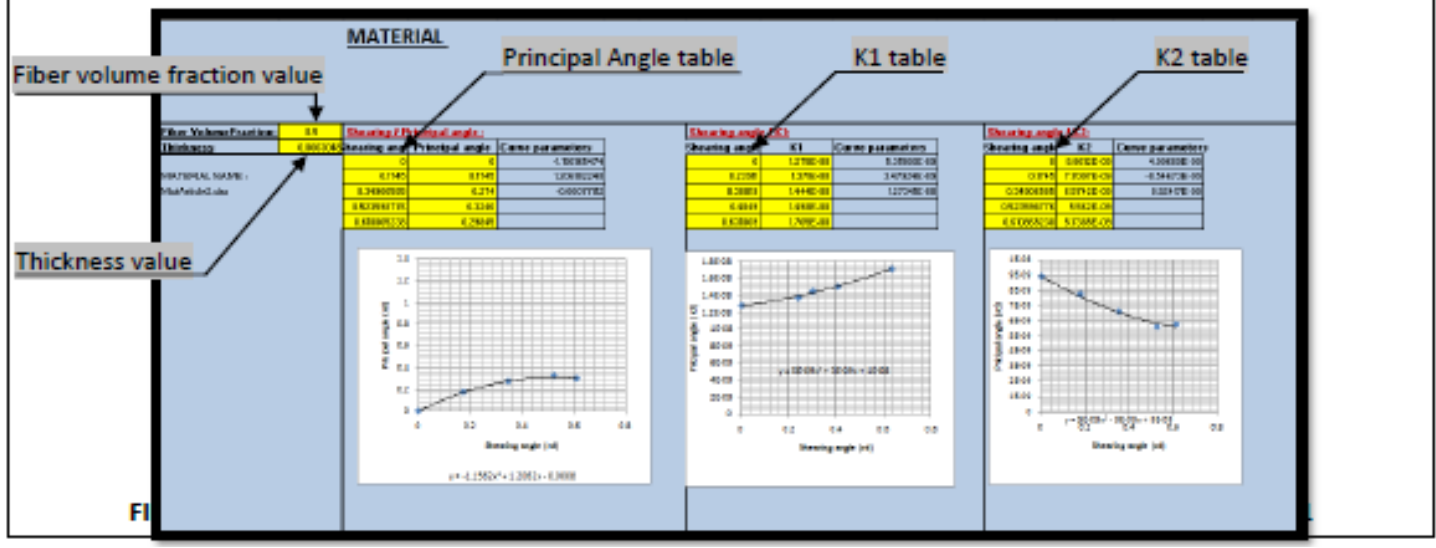




\section{FIBERSIM / UIMS TOOL: INTERFACE}

\section{FIBERSIM/LIMS TOOL}

\subsection{INTERFACE}

FIBER/LIMS Tool is composed by three modules:

- Input Module: Material database, Basic model and FiberSim Model.

- Conversion Module: 2D or 3D conversion and conversion options.

- Output Module: Final LIMS file creation for the flow simulation.

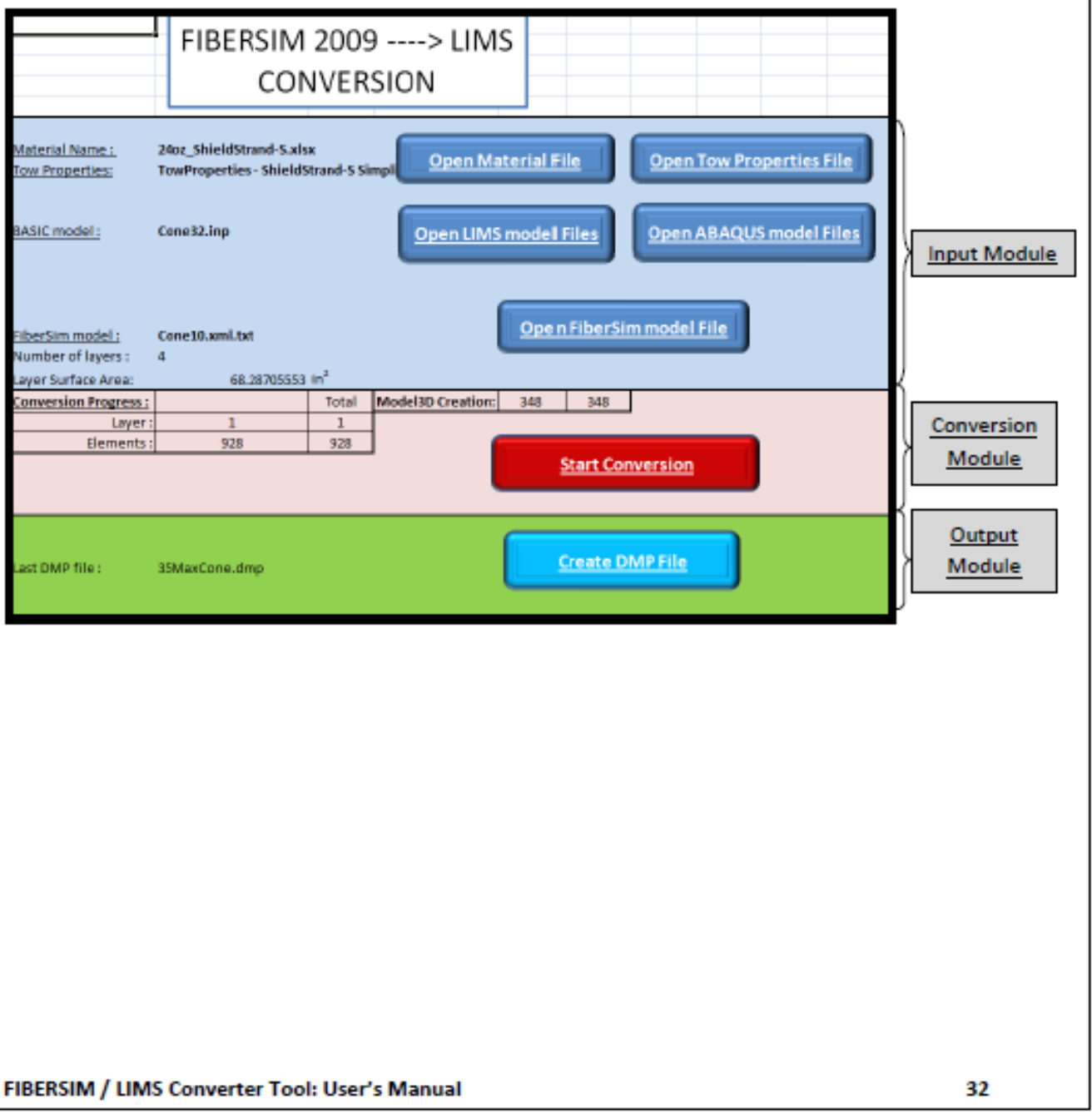




\section{FIBERSIM / LIMS TOOL: INNPUT MODULE}

\subsection{INPUT MODULE}

The FIBERSIM/LIMS Tool request three different inputs:

- The material characteristics: permeability evolution, ply thickness and initial fiber volume fraction.

- The Basic model: FEA model with 2D elements. (LIMS or ABAQUS)

- The Fibersim model file: XML file from FiberSim 2009 containing fibers orientation.

The following figure shows the Input module:

Name of actual material

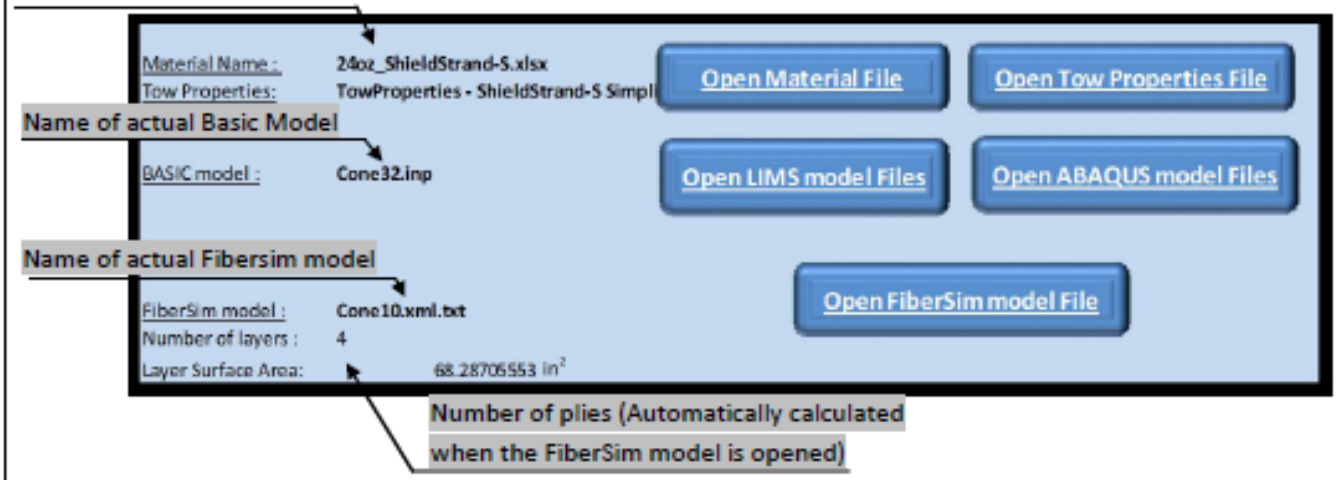

The Input module permits to load those inputs in FIBERSIM/LIMS Tool with the following procedure:

$>$ How to open a material file:

$\checkmark$ Click on the button "Open Material File".

$\checkmark$ Choose the material file (XIS) (This file has been created chapter 5)

$\checkmark$ Valid the selection with the button "Open"

$>$ How to open the basic model file: FIBERSIM/LIMS Tool can use a LIMS model or an ABAQUS Model.

$\checkmark$ Click on the button "Open LIMS model file" or "Open ABAQUS model file".

$\checkmark$ Choose the model file (dmp for LIMS or inp for ABAQUS)

$\checkmark$ Valid the selection with the button "Open"

$>$ How to open the Fibersim model file:

$\checkmark$ Click on the button "Open FiberSim model file".

$\checkmark$ Choose the model file (.xml)

$\checkmark$ Valid the selection with the button "Open" 


\section{FIBERSIM / UIMS TOOL: CONVERSION MODULE}

\subsection{CONVERSION MODULE}

The conversion module is composed by three elements:

- Conversion progression status: During the conversion the progression can be checked in this table

- Model3D progressions status: During the Model 3D creation the progression can be checked in this table.

- The conversion button.

After loading all inputs click on "Start Conversion" to launch the conversion Tool.

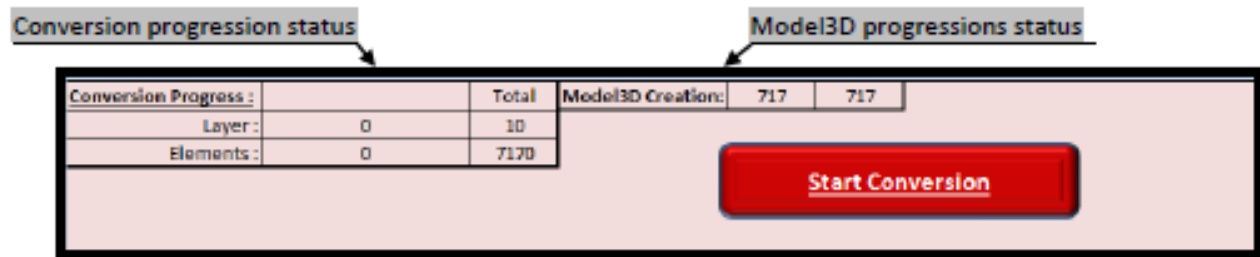

$>$ Conversion tool description:

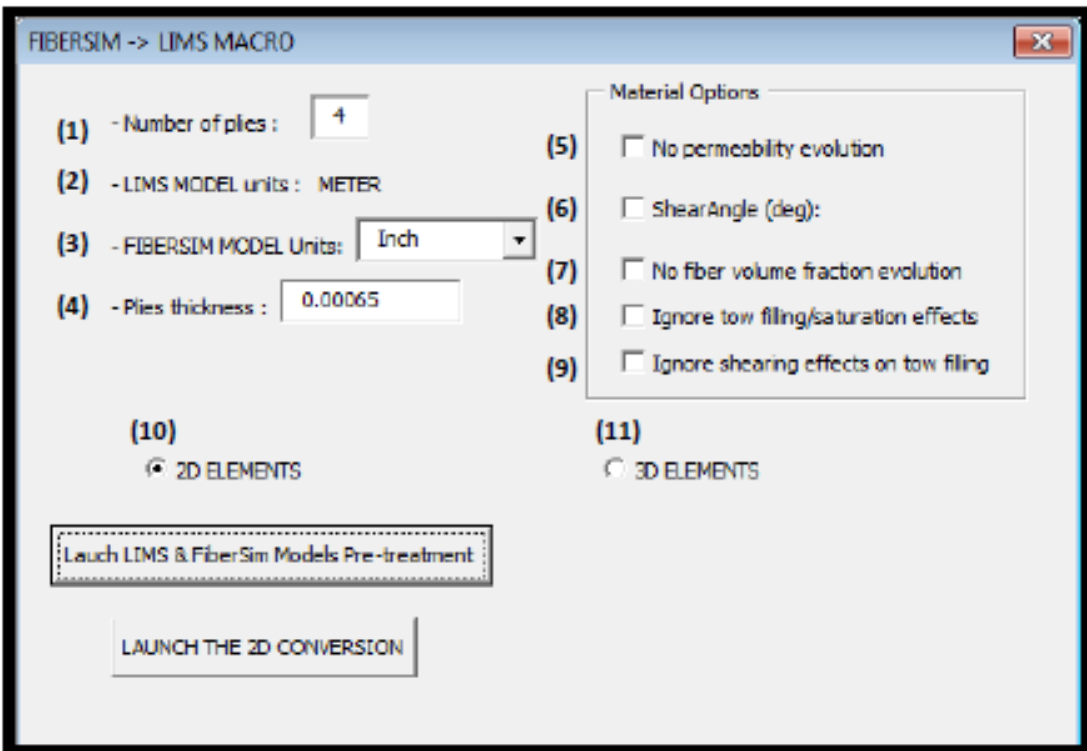


FIBERSIM / LIMS TOOL: CONVERSION MODULE

Models informations:

(1) : The number of plies. Automatic from FiberSim model. This value can be modified between 1 and the initial value. In this case only the $\mathrm{N}$ first layers will be used for the conversion.

(2) : Basic model units. (Meter)

(3) : FiberSim model unit choice. (Inch or Millimeter)

(4) : Thickness of one ply. Automatic from the material input. This value can be modified.

Materials option:

(5) : This option stops the bulk permeability evolution with the shearing angle.

(6) : This option set a constant value of the shearing angle. (Cannot be used with the option 5)

(7) : This option stops the fiber volume fraction evolution with the shearing angle.

(8) : This option ignores dual scale filling effects and cancels the creation of $1 \mathrm{D}$ tow elements.

(9) : This option stops the tow permeability evolution with the shearing angle

To obtain a simulation without shearing angle effect, use the options 5 and 7 .

Simulation Choice:

(10) : Simulation with 2D elements. (Detail Chapter 2):

$\checkmark$ Click on "Launch LIMS \& Fibersim model pre-treatment".

$\checkmark$ Click on "LAUNCH THE 2D CONVERSION".

$\checkmark$ Click "OK" when the windows "LIMS $\rightarrow$ FIBERSIM conversion finished" appears.

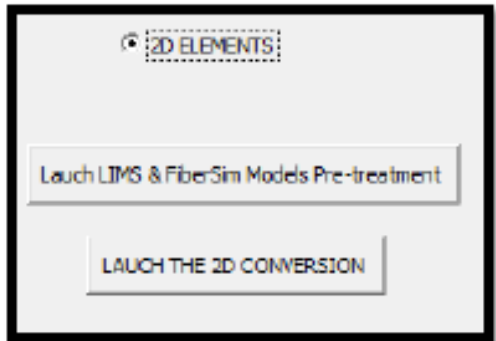




\section{FIBERSIM / LIMS TOOL: CONVERSION MODULE}

(11) : Simulation with 3D elements. (Detail Chapter 2):

$\checkmark$ Choose the 3D simulation option:

(12) : Define the number of elements layers through the thickness of the ply. The initial value is 1 , through the thickness only one element will be created for one ply. By adjusting this option you can create 3D model with more than 1 element by ply through the thickness.

(13) : Pick this option to create a 3D model with the ply by ply rule. (see Chapter 2.2)

(14) : Pick this option to create a 3D model with the average rule. (see Chapter 2.2)

$\checkmark$ Click on "MODEL 3D"

$\checkmark$ Click on "LAUNCH THE 3D CONVERSION"

$\checkmark$ Click "OK" when the windows "LIMS $\rightarrow$ FIBERSIM conversion finished" appears.

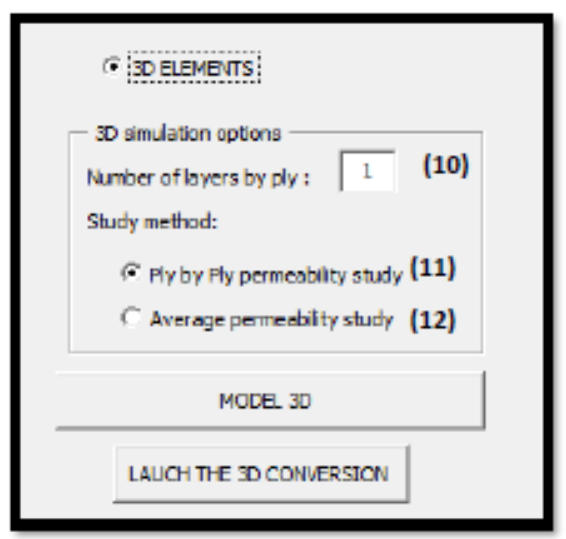


FIBERSIM / LIMS TOOL: OUTPUT MODULE

\subsection{OUPUT MODULE}

After the conversion, a new DMP file can be created for the simulation with LIMS. This file is created in the folder of LIMS/FIBERSIM Tool.

$\checkmark$ Click "Create DMP File".

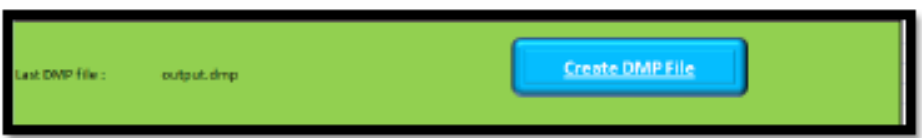

$>\underline{2 \mathrm{D} \text { conversion output: }}$

For 2D elements, the output tool proposes two options:

- Analysis with all the plies: All the plies will be used to create the DMP file. The averages rules describe in chapter 2 are used.

- Analysis of one ply: Only one ply will be use to create the DMP File. The number of the ply has to be entered.

$\checkmark$ Enter the output name

$\checkmark$ Tick "2D output"

$\checkmark$ Choose output options

$\checkmark$ Click "OK"

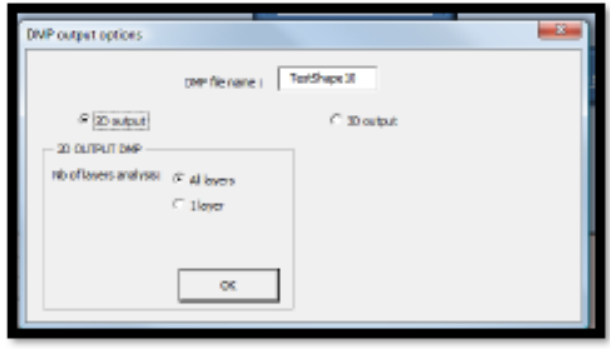

3D conversion output:

For 3D elements, the output tool creates a DMP file with all the plies.

$\checkmark$ Enter the output name

$\checkmark$ Tick "3D output"

$\checkmark$ Click "OK"

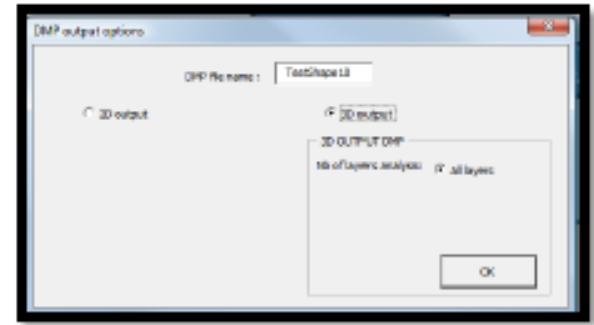









\section{QUESTIONS / ANSWERS}

\section{QUESTIONS / ANSWERS}

> OPENING MATERIAL, BASIC MODEL OR FIBERSIM MODEL:

When the action is cancelled by the button "CANCEL" an error appear: "Run-time error 1004".

Solution:

$\checkmark$ Click the button "End". The action will be canceled.

\section{OPENING FIBERSIM MODEL:}

After the choice of the file an error can appear: "Run-time error 1004".

Solution:

Click the button "End"

$\checkmark$ Re-open the file.

\section{MODEL 3D CREATION:}

The following enror can appears during the calculation of the 3D model: "Overflow".

This error potentially comes from the following reasons:

- The wrong unit has been chosen for the Abaqus file.

Solution:

$\checkmark$ Click the button "End"

$\checkmark$ Check the unit of the Abaqus file.

$\checkmark$ Re-open the Abaqus file.

$\checkmark$ Re-launch the 3D model creation.

- The wrong unit has been chosen for the Fibersim file.

Solution:

$\checkmark$ Click the button "End"

$\checkmark$ Check the unit of the Fibersim file

$\checkmark$ Change the unit of the Fibersim file in the conversion windows.

$\checkmark$ Re-launch the 3D model creation. 


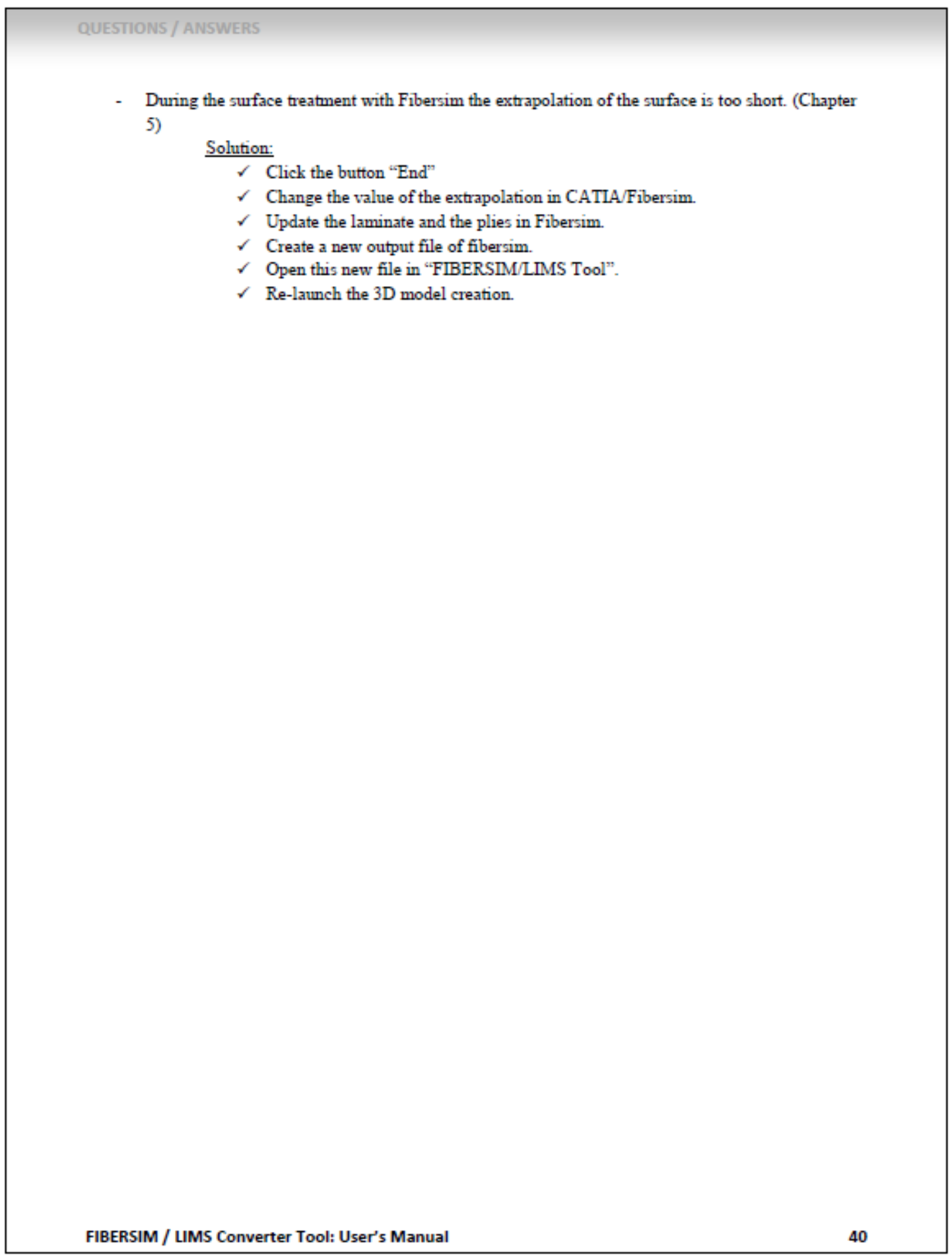




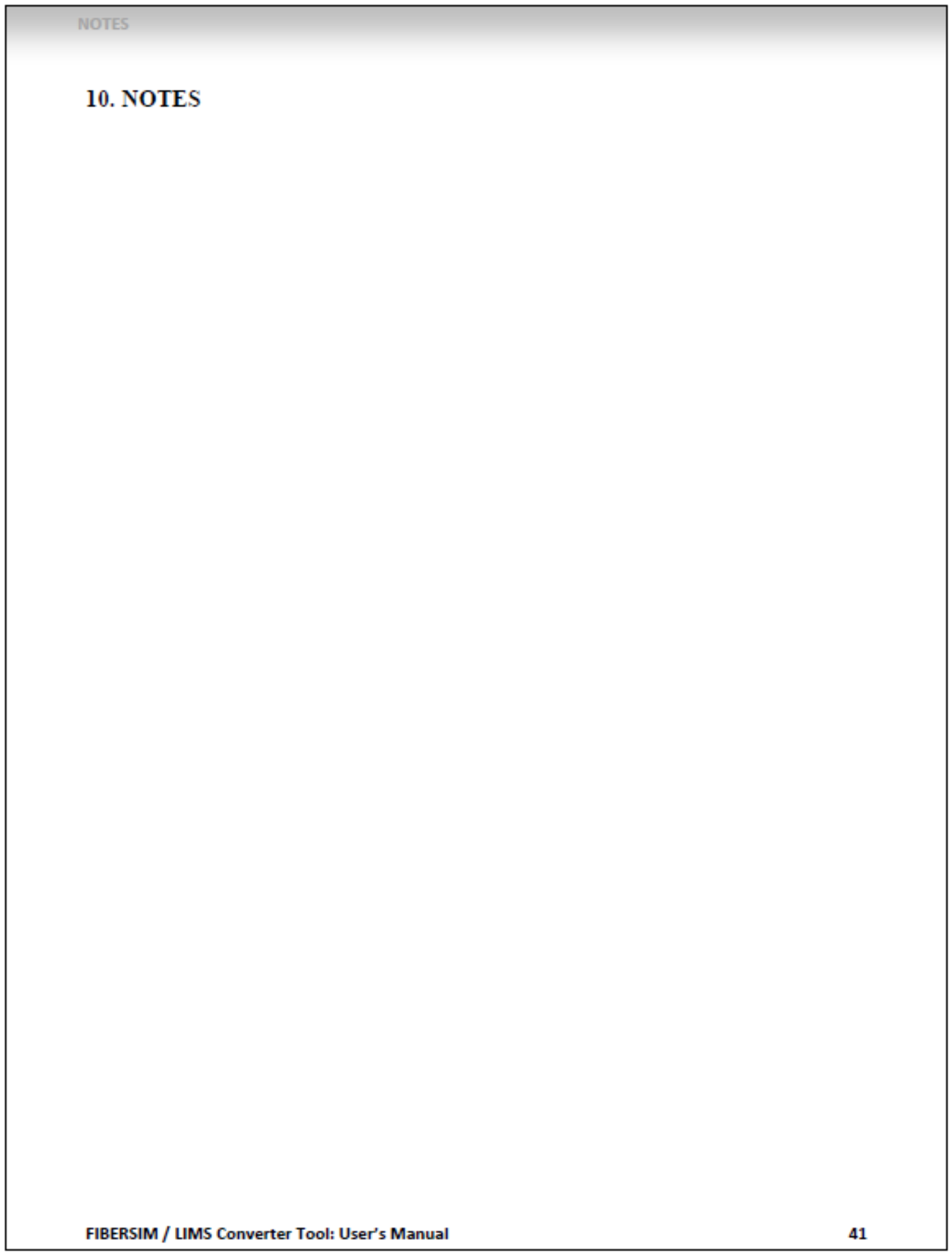

